\title{
Hyaline-spored chaetosphaeriaceous hyphomycetes from Thailand and China, with a review of the family Chaetosphaeriaceae
}

\author{
Lin CG ${ }^{1,2,3}$, McKenzie EHC ${ }^{4}$, Liu JK ${ }^{1,2 *}$, Jones EBG ${ }^{5}$, Hyde KD $^{3}$ \\ ${ }^{1}$ School of Life Science and Technology, University of Electronic Science and Technology of China, Chengdu 611731, \\ People's Republic of China \\ ${ }^{2}$ Guizhou Key Laboratory of Agricultural Biotechnology, Guizhou Academy of Agricultural Sciences, Guiyang 550006, \\ People's Republic of China \\ ${ }^{3}$ Center of Excellence in Fungal Research, Mae Fah Luang University, Chiang Rai 57100, Thailand \\ ${ }^{4}$ Manaaki Whenua Landcare Research, Private Bag 92170, Auckland, New Zealand \\ ${ }^{5}$ Dept. of Botany and Microbiology, College of Science, King Saud University, P.O Box 2455, Riyadh 11451, Kingdom \\ of Saudi Arabia
}

Lin CG, McKenzie EHC, Liu JK, Jones EBG, Hyde KD 2019 - Hyaline-spored chaetosphaeriaceous hyphomycetes from Thailand and China, with a review of the family Chaetosphaeriaceae. Mycosphere 10(1), 655-700, Doi 10.5943/mycosphere/10/1/14

\begin{abstract}
Chaetosphaeriaceae is a genus-rich, but taxonomically confused family and there is a need of more detailed studies on their asexual and sexual morphs and investigation of phylogenetic relationships to similar genera in other families. In this study, a survey of hyaline-spored chaetosphaeriaceous hyphomycetes yielded ten new taxa, including one new genus (Multiguttulispora) and nine new species (Dictyochaeta brevis, D. terminaliae, Kionochaeta castaneae, K. microspora, Menisporopsis breviseta, M. dushanensis, Multiguttulispora sympodialis, Tainosphaeria aseptata and T. monophialidica). In addition, Cryptophiale udagawae and Dictyochaeta simplex are described and illustrated. Support for the new taxa are provided by morphological comparison and DNA sequence data analyses. Phylogenetic analysis of SSU, ITS and LSU sequence data showed that Kionochaeta is polyphyletic and species are phylogenetically located in two groups (Kionochaeta 1 and Kionochaeta 2). In addition, a synopsis for Kionochaeta and Menisporopsis species, an updated phylogenetic tree and outline for Chaetosphaeriaceae are provided.
\end{abstract}

Key words - 10 new taxa - asexual morph - phylogeny - Sordariomycetes - Sordariomycetidae taxonomy

\section{Introduction}

The family Chaetosphaeriaceae was introduced by Locquin (1984) for Chaetosphaeria Tul. \& C. Tul., Loramyces W. Weston, Niesslia Auersw., Rhagadostoma Körb. and Zignoëlla Sacc., but was not validly published (Réblová et al. 1999). Réblová et al. (1999) re-described the family Chaetosphaeriaceae and accepted Ascocodinaea Samuels, Cand. \& Magni, Chaetosphaeria, Melanochaeta E. Müll., Harr \& Sulmont, Melanopsammella Höhn., Porosphaerella E. Müll. \& Samuels, Porosphaerellopsis Samuels \& E. Müll. and Striatosphaeria Samuels \& E. Müll. Presently, 38 genera are accepted within the family Chaetosphaeriaceae (Maharachchikumbura et al. 2016, Wijayawardene et al. 2018). 
Cryptophiale Piroz. was introduced by Pirozynski (1968) to accommodate C. kakombensis Piroz. and C. udagawae Piroz. \& Ichinoe and subsequently 20 species are accepted (Whitton et al. 2012). Cryptophiale is most similar to Cryptophialoidea Kuthub. \& Nawawi, but differs by its conidiogenous cells encircling the conidiophores, protected by shield cells, which are on one side of the conidiophore and are not covered by sterile shield cells (Whitton et al. 2012).

Dictyochaeta was established by Spegazzini (1923) to accommodate D. fuegiana Speg. It is characterized by unbranched or branched, brown conidiophores with or without unbranched setae, monophialidic or polyphialidic, brown conidiogenous cells which produce aseptate and hyaline conidia. There are 104 epithets listed in Index Fungorum (July, 2019). Kuthubutheen \& Nawawi (1991b) provided a key to Dictyochaeta and Codinaea species.

Kionochaeta P.M. Kirk \& B. Sutton was introduced by Kirk \& Sutton (1985) when they amended the genus Chaetopsina Rambelli. Kionochaeta is similar to Zanclospora, but differs by its conidiogenous cells borne on variously arranged branches of conidiophores, however, the conidiogenous cells are sessile, arranged in whorls on a conidiophore in Zanclospora (Kirk \& Sutton 1985).

Menisporopsis S. Hughes was established by Hughes (1952) to accommodate M. theobromae S. Hughes. Liu et al. (2016) provided a detailed description of M. theobromae along with DNA sequence data and phylogeny for the first time for this genus. There are 11 epithets listed in Index Fungorum (July, 2019). Keys to the Menisporopsis species were provided by Tsui et al. (1999) and Castañeda Ruíz et al. (2001).

Tainosphaeria F.A. Fernández \& Huhndorf was introduced by Fernández \& Huhndorf (2005) with T. crassiparies F.A. Fernández \& Huhndorf as the type species, and placed within the family Chaetosphaeriaceae. Three species are accepted within this genus (Fernández \& Huhndorf 2005, Liu et al. 2016, Lu et al. 2016). Its placement in Chaetosphaeriaceae was confirmed by phylogenetic analyses (Fernández et al. 2006, Liu et al. 2016, Lu et al. 2016).

During a survey of hyphomycetes in China and Thailand, several hyaline-spored chaetosphaeriaceous species were collected. In this study, we introduce one new genus Multiguttulispora with its type species $M$. sympodialis, two new Dictyochaeta species (D. brevis and $D$. terminaliae), two new Kionochaeta species (K. castaneae and K. microspora), two new Menisporopsis species ( $M$. breviseta and $M$. dushanensis), two new Tainosphaeria species ( $T$. aseptata and $T$. monophialidica) along with two previously described species, Cryptophiale udagawae and Dictyochaeta simplex based on morphology and phylogenetic analyses.

\section{Materials \& Methods}

\section{Collection and isolation of fungi}

Dead wood from a variety of plants in forests were collected in Krabi Province, Thailand and Guizhou Province, China. Samples were taken to the laboratory in Zip-lock plastic bags for examination. The specimens were incubated in sterile moist chambers and examined using a Motic SMZ 168 series microscope. Fungi were removed with a needle and placed in a drop of distilled water on a slide for morphological study. Photomicrographs of fungal structures were captured with a Canon 600D digital camera attached to a Nikon ECLIPSE Ni compound microscope. All measurements were made using the Tarosoft (R) Image FrameWork program (Liu et al. 2010). Photo-plates were made with Adobe Photoshop CS3 (Adobe Systems, USA). Isolation onto potato dextrose agar (PDA) was performed by the single spore isolation method (Chomnunti et al. 2014). Dried material was deposited in the Herbarium of Mae Fah Luang University (MFLU), Chiang Rai, Thailand and herbarium of Kunming Institute of Botany, Chinese Academy of Sciences (HKAS), Kunming, China. Cultures were deposited at Mae Fah Luang University Culture Collection (MFLUCC), Chiang Rai, Thailand and Guizhou Culture Collection, China (GZCC). Faces of Fungi and Index Fungorum numbers were registered (Jayasiri et al. 2015, Index Fungorum 2019). 


\section{DNA extraction, PCR amplification and sequencing}

Genomic DNA was extracted from pure fungal mycelium grown on PDA or malt extract agar (MEA) at room temperature using the Fungal gDNA Kit (BioMIGA, USA) according to the manufacturer's instructions. The internal transcribed spacer region of ribosomal DNA (ITS), large subunit nuclear ribosomal DNA (LSU) and small subunit nuclear ribosomal DNA (SSU) genes regions were amplified via polymerase chain reaction (PCR) using the following primers: ITS5 and ITS4 (White et al. 1990) for ITS, LR0R and LR5 (Vilgalys \& Hester 1990) for LSU, and NS1 and NS4 for SSU (White et al. 1990). The PCR products were sequenced with the same primers. The PCR amplification was performed in a $25 \mu \mathrm{L}$ reaction volume containing $12.5 \mu \mathrm{L}$ of $2 \times$ Power Taq PCR MasterMix (a premix and ready to use solution, including 0.1 Units/ $\mu \mathrm{L}$ Taq DNA Polymerase, $500 \mu \mathrm{M}$ dNTP Mixture each [dATP, dCTP, dGTP, dTTP], $20 \mathrm{mM}$ Tris-HCl pH 8.3, $100 \mathrm{Mm} \mathrm{KCl,}$ $3 \mathrm{mM} \mathrm{MgCl} 2$, stabilizer and enhancer), $1 \mu \mathrm{L}$ of each primer $(10 \mu \mathrm{M}), 1 \mu \mathrm{L}$ genomic DNA extract and $9.5 \mu \mathrm{L}$ deionised water. The PCR thermal cycle program of ITS and LSU were: initially $94{ }^{\circ} \mathrm{C}$ for $3 \mathrm{~min}$., followed by 35 cycles of denaturation at $94{ }^{\circ} \mathrm{C}$ for $30 \mathrm{~s}$, annealing at $55{ }^{\circ} \mathrm{C}$ for $50 \mathrm{~s}$, elongation at $72{ }^{\circ} \mathrm{C}$ for $1 \mathrm{~min}$., and final extension at $72{ }^{\circ} \mathrm{C}$ for $10 \mathrm{~min}$.

\section{Phylogenetic analyses}

Original sequences were checked using BioEdit version 7.0.5.3 (Hall 1999), along with reference sequences originated from previous publications. The remaining homogenous sequences were obtained by BLAST searches (Altschul et al. 1990) from GenBank. All sequences used in this study are listed in Table 1. Alignments for each locus were done in MAFFT v7.307 online version (Katoh \& Standley 2016) and manually verified in MEGA 6.06 (Tamura et al. 2013). Conserved blocks were selected from the initial alignments with Gblocks 0.91b (Castresana 2000). The interleaved NEXUS files for Bayesian inference analyses were formatted with AliView v1.19beta1k (Larsson 2014). Bayesian inference (BI), maximum parsimony (MP) and maximum likelihood (ML) were used for phylogenetic analyses. For Bayesian inference analysis, the best model of evolution was determined using MrModeltest v2 (Nylander 2004). Bayesian inference analysis was done with MrBayes v 3.2.6 (Ronquist et al. 2012). Maximum parsimony analysis was performed in PAUP*4.0b10 (Swofford 2002). Maximum likelihood analysis was performed in raxmlGUI v 1.3.1 (Silvestro \& Michalak 2012). Phylogenetic trees were drawn with TreeView 1.6.6 (Page 1996) or FigTree v1.4.3 (Rambaut 2017).

\section{Results}

\section{Molecular phylogeny}

The combined LSU and ITS sequence dataset included 214 taxa (ingroup) and two outgroup taxa with a total of 1670 characters (954 characters for LSU, 716 characters for ITS) after alignment including the gaps, of which 722 were parsimony informative, 228 were parsimonyuninformative, and 720 characters were constant. The tree was rooted with Gelasinospora tetrasperma (AFTOL-ID 1287) and Sordaria fimicola (CBS 508.50). The ML and BI analyses based on combined LSU and ITS sequence data provided similar tree topologies, and the result of ML analysis with a final likelihood value of -32807.818894 is shown in Fig. 1. The matrix had 1149 distinct alignment patterns, with $29.85 \%$ undetermined characters or gaps. Estimated base frequencies were: $\mathrm{A}=0.233314, \mathrm{C}=0.262649, \mathrm{G}=0.309421, \mathrm{~T}=0.194616$; substitution rates $\mathrm{AC}$ $=1.000000, \mathrm{AG}=3.741518, \mathrm{AT}=1.000000, \mathrm{CG}=1.000000, \mathrm{CT}=3.741518, \mathrm{GT}=1.000000$; gamma distribution shape parameter $\alpha=0.303701$. For the Bayesian analysis, two parallel runs with six chains were run for 100000000 generations and trees were sampled every 10000th generation and the run was stopped automatically when standard deviation of split frequencies fell below 0.01 . The run automatically stopped at 31790000 generations, resulting in 6360 trees from two runs of which 4770 trees were used to calculate the posterior probabilities (each run resulted in 3180 trees of which 2385 trees were sampled). 
Eleven isolates of hyaline-spored chaetosphaeriaceous hyphomycetes from China and Thailand were identified in the family Chaetosphaeriaceae and located in the genera Cryptophiale, Dictyochaeta, Kionochaeta, Menisporopsis, Tainosphaeria and the new genus Multiguttulispora (Fig. 1).

On the ML tree (Fig. 1), Chaetosphaeria species are located in 14 different groups, and some of them grouped with the asexual genera, e.g. Chloridium (group Chloridium 3), Dictyochaeta (group Dictyochaeta 4 and Dictyochaeta 5), Exserticlava (group Chaetosphaeria 3), Menispora (group Menispora) and Umbrinosphaeria (group Chaetosphaeria 3).

Dictyochaeta species are phylogenetically located in five different groups (Fig. 1), which are the same as reported by Liu et al. (2016), Perera et al. (2016), Hernández-Restrepo et al. (2017), Tibpromma et al. (2018), Yang et al. (2018) and Luo et al. (pers. comm.).

To analyse the genus Kionochaeta, a combined SSU, ITS and LSU sequence dataset was also analyzed (Fig. 2). This dataset included 33 taxa (ingroup) and one outgroup taxon with a total of 2480 characters (1036 characters for SSU, 602 characters for ITS, 842 characters for LSU) after alignment including the gaps, of which 524 were parsimony informative, 307 were parsimonyuninformative, and 1649 characters were constant. The tree was rooted with Gelasinospora tetrasperma (AFTOL-ID 1287). The MP, ML and BI analyses based on combined SSU, ITS and LSU sequence data provided similar tree topologies, and the result of ML analysis with a final likelihood value of -14301.378740 is shown in Fig. 2. The matrix had 922 distinct alignment patterns, with $20.33 \%$ undetermined characters or gaps. Estimated base frequencies were: $\mathrm{A}=$ $0.241157, \mathrm{C}=0.247000, \mathrm{G}=0.286422, \mathrm{~T}=0.225421$; substitution rates $\mathrm{AC}=1.270116, \mathrm{AG}=$ 1.760629, $\mathrm{AT}=1.010454, \mathrm{CG}=0.828899, \mathrm{CT}=5.543715, \mathrm{GT}=1.000000$; gamma distribution shape parameter $\alpha=0.428282$. For the Bayesian analysis, two parallel runs with six chains were run for 1000000 generations and trees were sampled every 100th generation, resulting in 20002 trees from two runs of which 15002 trees were used to calculate the posterior probabilities (each run resulted in 10001 trees of which 7501 trees were sampled).

Our two newly collected Kionochaeta isolates grouped together with another three Kionochaeta isolates (K. ramifera (Matsush.) P.M. Kirk \& B. Sutton (JCM9756) and K. spissa P.M. Kirk \& B. Sutton (JCM9817 and JCM9818)) (Fig. 2), however, this group is separate from the group comprising the ex-type strain of K. ivoriensis (Rambelli \& Lunghini) P.M. Kirk \& B. Sutton (CBS 374.76). Kionochaeta is similar to Zanclospora, but they are phylogenetically different (Fig. 2).

\section{Taxonomy}

Cryptophiale udagawae Piroz. \& Ichinoe, in Pirozynski, Can. J. Bot. 46: 1126 (1968)

Fig. 3

Facesoffungi number: FoF06284

Saprobic on decaying wood. Asexual morph: Colonies on plant substrate effuse, hairy, scattered, glistening. Mycelium mostly immersed, partially superficial. Conidiophores macronematous, mononematous, setiform, scattered, erect, straight or slightly curved, dark brown, smooth, thick-walled, septate below the branches, 150-290 × 10-33 $\mu \mathrm{m}, 1-2$ times dichotomously branched towards the apex and generally above the fertile region; terminal branches dark brown, 9.5-51.5 $\mu \mathrm{m}$ long, $2.9-6.2 \mu \mathrm{m}$ wide at the base, acutely pointed above. Fertile region from middle up to the first dichotomy, 36.5-92 × 10-17 $\mu \mathrm{m}$, consisting of two rows of phialides one on each side of the conidiophore with each cell narrowly ellipsoid, covered by sterile shield cells. Conidiogenous cells not observed. Conidia produced in slime and adhering to the fertile region, hyaline, smooth, clavate, aseptate, basal end rounded, with a short appendage at the base, 19.9-28.1 $\times 1.6-2.8 \mu \mathrm{m}(\mathrm{av} .=24.9 \times 2.05 \mu \mathrm{m}, \mathrm{n}=30)$. Sexual morph: Undetermined.

Material examined - CHINA, Guizhou Province, Qiannan Buyi Miao Autonomous Prefecture, Dushan County, Guizhou Zilinshan National Forest Park (Shengou District), unnamed road, on decaying wood, 6 July 2018, Chuan-Gen Lin, DS 1-36 (MFLU 19-0209, HKAS 105133), living culture GZCC 18-0047. 
Table 1 GenBank accession numbers of isolates included in this study.

\begin{tabular}{|c|c|c|c|c|c|c|}
\hline Taxa & Isolate $^{\mathrm{a}, \mathrm{b}}$ & Status $^{c}$ & LSU & ITS & SSU & Note \\
\hline Adautomilanezia caesalpiniae & $\begin{array}{l}\text { HUEFS } 216632 \text { = } \\
\text { LAMIC010212 = CC-LAMIC } \\
\text { 102/12 }\end{array}$ & $\mathrm{T}$ & NG_058594 & NR_153560 & $-\mathrm{d}^{\mathrm{d}}$ & \\
\hline Anacacumisporium appendiculatum & HMAS 245593 & $\mathrm{~T}$ & КT001553 & КT001555 & - & \\
\hline Anacacumisporium appendiculatum & HMAS 245602 & & КT001554 & КT001556 & - & \\
\hline Bahusutrabeeja dwaya & CBS 261.77 = JCM 6357 & $\mathrm{~T}$ & MH872829 & MH861059 & - & \\
\hline Brunneodinemasporium brasiliense & CBS 112007 & $\mathrm{~T}$ & JQ889288 & JQ889272 & - & \\
\hline Brunneodinemasporium jonesii & GZCC 16-0050 & $\mathrm{T}$ & KY026055 & KY026058 & - & \\
\hline Cacumisporium capitulatum & FMR 11339 & & HF677190 & HF677176 & - & $\begin{array}{l}\text { Sexual morph Chaetosphaeria } \\
\text { decastyla }\end{array}$ \\
\hline Cacumisporium capitulatum & SMH 3766 & & AY017374 & - & - & $\begin{array}{l}\text { Sexual morph Chaetosphaeria } \\
\text { decastyla }\end{array}$ \\
\hline Chaetosphaeria acutata & CBS 101312 & & AF178553 & AF178553 & - & \\
\hline Chaetosphaeria albida & PDD 92537 & $\mathrm{~T}$ & NG_058756 & NR_119666 & - & \\
\hline Chaetosphaeria biapiculata & SMH 3074 & & AF466065 & - & - & \\
\hline Chaetosphaeria caesariata & SMH 2794 & & AF466060 & - & - & $\begin{array}{l}\text { Now known as Stanjehughesia } \\
\text { hormiscioides }\end{array}$ \\
\hline Chaetosphaeria callimorpha & CBS 525.88 & & AF178555 & AF178555 & - & \\
\hline Chaetosphaeria callimorpha & SMH 2791 & & AF466062 & - & - & \\
\hline Chaetosphaeria capitata & SMH 3239 & & AF466061 & - & - & \\
\hline Chaetosphaeria chalaroides & SMH 2018 & & AY017372 & - & - & \\
\hline Chaetosphaeria chalaroides & SMH 2223 & & AF466063 & - & - & \\
\hline Chaetosphaeria chlorotunicata & SMH 1565 & & AF466064 & - & - & \\
\hline Chaetosphaeria ciliata & CBS 122131 & $\mathrm{~T}$ & MH874726 & MH863180 & & Asexual morph Menispora ciliata \\
\hline Chaetosphaeria ciliata & ICMP 18253 & & GU180637 & - & GU180614 & Asexual morph Menispora ciliata \\
\hline Chaetosphaeria conirostris & SMH 2183 & & AF466066 & - & - & \\
\hline Chaetosphaeria cubensis & SMH 3258 & & AF466067 & - & - & \\
\hline Chaetosphaeria curvispora & CBS 125555 & & MH875040 & MH863562 & - & \\
\hline Chaetosphaeria curvispora & CBS 125782 & & MH875199 & MH863736 & - & \\
\hline Chaetosphaeria curvispora & ICMP 18255 & & GU180636 & - & - & \\
\hline Chaetosphaeria cylindrospora & SMH 3568 & $\mathrm{~T}$ & AY017373 & - & - & \\
\hline Chaetosphaeria decastyla & SMH 2629 & & AF466068 & - & - & \\
\hline Chaetosphaeria dilabens & CBS 712.88 & & AF178557 & AF178557 & - & \\
\hline
\end{tabular}


Table 1 Continued.

\begin{tabular}{|c|c|c|c|c|c|c|}
\hline Taxa & Isolate $^{a, b}$ & Status $^{c}$ & LSU & ITS & SSU & Note \\
\hline Chaetosphaeria dilabens & CBS 734.83 & & MH873395 & MH861683 & - & \\
\hline Chaetosphaeria fennica & CBS 101641 & & AF178562 & AF178562 & - & \\
\hline Chaetosphaeria fuegiana & CBS 114553 & & MH874528 & - & - & \\
\hline Chaetosphaeria fusiformis & CBS 101429 & & AF178554 & AF178554 & - & \\
\hline Chaetosphaeria garethjonesii & MFLUCC 15-1012 & $\mathrm{T}$ & NG_059017 & NR_154840 & - & \\
\hline Chaetosphaeria hebetiseta & MR 938 & & AF178549 & AF178549 & - & \\
\hline Chaetosphaeria hebetiseta & SMH 2729 & & AF466069 & AY906955 & - & \\
\hline Chaetosphaeria inaequalis & MR 1450 & & AF178564 & AF178564 & - & \\
\hline Chaetosphaeria innumera & MR 1175 & & AF178551 & AF178551 & - & \\
\hline Chaetosphaeria innumera & SMH 2748 & & AY017375 & AY906956 & - & \\
\hline Chaetosphaeria jonesii & MFLUCC 15-1015 & $\mathrm{T}$ & KY212761 & NR_154841 & - & \\
\hline Chaetosphaeria lateriphiala & SMH 2629-1 & & AF466070 & - & - & \\
\hline Chaetosphaeria lateriphiala & SMH 3294 & & AF466071 & - & - & \\
\hline Chaetosphaeria lateriphiala & SMH 3320 & & AF466072 & - & - & \\
\hline Chaetosphaeria lentomita & MR 1265 & & AF178548 & AF178548 & - & \\
\hline Chaetosphaeria lignomollis & SMH 3015 & & AF466073 & EU037896 & - & \\
\hline Chaetosphaeria longiseta & SMH 1725 & & AF279416 & - & - & \\
\hline Chaetosphaeria longiseta & SMH 3854 & & AF279417 & - & - & \\
\hline Chaetosphaeria luquillensis & SMH 2973 & & AF466074 & - & - & \\
\hline Chaetosphaeria mangrovei & MCD 069 & $\mathrm{~T}$ & MG813820 & MG813821 & - & \\
\hline Chaetosphaeria metallicans & PDD 92539 & $\mathrm{~T}$ & NG_058757 & NR_119668 & - & \\
\hline Chaetosphaeria minuta & SMH 3396 & & AF466075 & - & - & \\
\hline Chaetosphaeria myriocarpa & CBS 116.57 & & MH869208 & MH857668 & - & \\
\hline Chaetosphaeria myriocarpa & CBS 141.53 & & MH868672 & MH857137 & - & \\
\hline Chaetosphaeria myriocarpa & CBS 143389 & & MH107931 & MH107883 & - & \\
\hline Chaetosphaeria myriocarpa & CBS 241.75B & & MH872648 & MH860912 & - & \\
\hline Chaetosphaeria myriocarpa & CBS 264.76 & & AF178552 & AF178552 & - & \\
\hline Chaetosphaeria myriocarpa & MUCL 34784 & & AF466076 & - & - & \\
\hline Chaetosphaeria panamensis & MFLUCC 15-1011 & & KY212760 & KY212752 & - & $\begin{array}{l}\text { Asexual morph Craspedodidymum- } \\
\text { like }\end{array}$ \\
\hline Chaetosphaeria preussii & CBS 262.76 & & AF178561 & AF178561 & - & \\
\hline Chaetosphaeria pygmaea & UPSC 2523 & & AF466077 & - & - & \\
\hline
\end{tabular}


Table 1 Continued.

\begin{tabular}{|c|c|c|c|c|c|c|}
\hline Taxa & Isolate $^{\mathrm{a}, \mathrm{b}}$ & Status $^{c}$ & LSU & ITS & SSU & Note \\
\hline Chaetosphaeria pygmaea & MR 1365 & & AF178545 & AF178545 & - & \\
\hline Chaetosphaeria raciborskii & SMH 2017 & & AF466078 & AY906949 & - & $\begin{array}{l}\text { Asexual morph Craspedodidymum- } \\
\text { like }\end{array}$ \\
\hline Chaetosphaeria raciborskii & SMH 3119 & & AY436402 & AY906953 & - & $\begin{array}{l}\text { Asexual morph Craspedodidymum- } \\
\text { like }\end{array}$ \\
\hline Chaetosphaeria rivularia & CBS 127686 & $\mathrm{~T}$ & KR347357 & KR347356 & - & \\
\hline Chaetosphaeria spinosa & SMH 2754 & & AF466079 & - & - & \\
\hline Chaetosphaeria sylvatica & SMH 2893 & & AF279419 & - & - & \\
\hline Chaetosphaeria tropicalis & SMH 1267 & & AF279418 & - & - & \\
\hline Chaetosphaeria tropicalis & SMH 2250 & & AF466080 & - & - & \\
\hline Chloridium aquaticum & HKAS 96226 & $\mathrm{~T}$ & MH476567 & MH476570 & MH476573 & \\
\hline Chloridium aseptatum & MFLUCC 11-0216 & $\mathrm{T}$ & MH476568 & NR_158365 & MH476574 & \\
\hline Chloridium botryoideum & CBS 131270 & & MH877338 & - & - & \\
\hline $\begin{array}{l}\text { Chloridium botryoideum var. } \\
\text { botryoideum }\end{array}$ & CBS 259.76 & & MH878530 & - & - & \\
\hline $\begin{array}{l}\text { Chloridium botryoideum var. } \\
\text { botryoideum }\end{array}$ & CBS 246.76 & & MH878531 & - & - & \\
\hline Chloridium chloroconium & FMR 11940 & & KY853495 & KY853435 & - & $\begin{array}{l}\text { Before known as Gonytrichum } \\
\text { chlamydosporoides var. simile }\end{array}$ \\
\hline Chloridium lignicola & CBS 143.54 & IT & MH857273 & MH857273 & - & $\begin{array}{l}\text { Before known as Bisporomyces } \\
\text { lignicola }\end{array}$ \\
\hline Chloridium sp. & HGUP1806 & & MK372068 & MK372070 & - & \\
\hline Chloridium sp. & HGUP1805 & & MK372067 & MK372069 & - & \\
\hline Chloridium virescens & MR 1148 & & AF178550 & AF178550 & - & \\
\hline Chloridium virescens & NRRL 37636 & & GU183124 & GU183124 & - & \\
\hline $\begin{array}{l}\text { Chloridium virescens var. } \\
\text { caudigerum }\end{array}$ & CBS 152.53 & & MH868678 & MH857142 & - & \\
\hline $\begin{array}{l}\text { Chloridium virescens var. } \\
\text { caudigerum }\end{array}$ & CBS 141.54 & & MH868805 & MH857272 & - & \\
\hline $\begin{array}{l}\text { Chloridium virescens var. } \\
\text { caudigerum }\end{array}$ & CBS 126073 & & MH875524 & MH864068 & - & \\
\hline $\begin{array}{l}\text { Chloridium virescens var. } \\
\text { chlamydosporum }\end{array}$ & CBS 345.67 & & MH870689 & MH858992 & - & $\begin{array}{l}\text { Before known as Bisporomyces } \\
\text { chlamydosporus, now known as } \\
\text { Chaetosphaeria vermicularioides }\end{array}$ \\
\hline
\end{tabular}


Table 1 Continued.

\begin{tabular}{|c|c|c|c|c|c|c|}
\hline Taxa & Isolate $^{\mathrm{a}, \mathrm{b}}$ & Status $^{c}$ & LSU & ITS & SSU & Note \\
\hline $\begin{array}{l}\text { Chloridium virescens var. } \\
\text { chlamydosporum }\end{array}$ & CBS 126074 & & MH875525 & MH864069 & - & $\begin{array}{l}\text { Before known as Bisporomyces } \\
\text { chlamydosporus, now known as } \\
\text { Chaetosphaeria vermicularioides }\end{array}$ \\
\hline $\begin{array}{l}\text { Chloridium virescens var. } \\
\text { chlamydosporum }\end{array}$ & CBS 142.61 & & MH869557 & - & - & $\begin{array}{l}\text { Before known as Bisporomyces } \\
\text { chlamydosporus, now known as } \\
\text { Chaetosphaeria vermicularioides }\end{array}$ \\
\hline $\begin{array}{l}\text { Chloridium virescens var. } \\
\text { chlamydosporum }\end{array}$ & CBS 114.41 & $\mathrm{~T}$ & MH867576 & - & - & $\begin{array}{l}\text { Before known as Bisporomyces } \\
\text { chlamydosporus, now known as } \\
\text { Chaetosphaeria vermicularioides }\end{array}$ \\
\hline Chloridium virescens var. virescens & CBS 239.75B & & MH878291 & - & - & \\
\hline Codinaea lambertiae & CBS 143419 = CPC 32289 & $\mathrm{~T}$ & NG_059053 & NR_156389 & - & \\
\hline Codinaea pini & CBS 138866 & $\mathrm{~T}$ & NG_058902 & NR_137943 & - & \\
\hline Codinaeopsis gonytrichoides & CBS 593.93 & & AF178556 & AF178556 & - & \\
\hline Conicomyces pseudotransvaalensis & HHUF 29956 = GS20 & $\mathrm{T}$ & LC001708 & NR_138015 & - & \\
\hline Cryptophiale hamulata & MFLUCC 18-0098 & & MG386756 & - & MG386757 & \\
\hline Cryptophiale udagawae & MFLUCC 18-0422 & & MH758211 & MH758198 & MH758205 & \\
\hline Cryptophiale udagawae & MFLUCC 18-0428 & & MH758210 & MH758197 & MH758204 & \\
\hline Cryptophiale udagawae & GZCC 18-0047 & & MN104619 & MN104608 & MN104628 & \\
\hline Cryptophialoidea fasciculata & MFLUCC 17-2119 & & MH758208 & MH758195 & MH758202 & \\
\hline Dendrophoma cytisporoides & CBS 223.95 & $\mathrm{~T}$ & JQ889289 & JQ889273 & - & \\
\hline Dictyochaeta aquatica & MFLUCC 15-0983 & $\mathrm{T}$ & MH476569 & NR_158452 & MH476575 & \\
\hline Dictyochaeta assamica & CBS 242.66 & & MH870426 & MH858788 & - & \\
\hline Dictyochaeta brevis & GZCC 18-0096 & $\mathbf{T}$ & MN104625 & MN104614 & MN104634 & \\
\hline Dictyochaeta fuegiana & FMR 13126 & & KY853500 & KY853440 & - & $\begin{array}{l}\text { Sexual morph Chaetosphaeria } \\
\text { fuegiana }\end{array}$ \\
\hline Dictyochaeta fuegiana & ICMP 15153 & & EF063574 & - & - & $\begin{array}{l}\text { Sexual morph Chaetosphaeria } \\
\text { fuegiana }\end{array}$ \\
\hline Dictyochaeta mimusopis & CBS 143435 & & MH107935 & MH107888 & - & \\
\hline Dictyochaeta pandanicola & $\begin{array}{l}\text { KUMCC 16-0153 = MFLUCC } \\
\text { 17-0563 }\end{array}$ & $\mathrm{T}$ & MH376710 & МН388338 & МН388307 & \\
\hline Dictyochaeta septata & CBS 143386 & & MH107936 & MH107889 & - & \\
\hline Dictyochaeta siamensis & MFLUCC 15-0614 & $\mathrm{T}$ & KX609952 & KX609955 & - & \\
\hline Dictyochaeta siamensis & MFLUCC 16-0371 & & MH376711 & МН388339 & МН388308 & \\
\hline Dictyochaeta simplex & CBS 966.69 & & AF178559 & AF178559 & - & Before known as Codinaea simplex \\
\hline
\end{tabular}


Table 1 Continued.

\begin{tabular}{|c|c|c|c|c|c|c|}
\hline Taxa & Isolate $^{a, b}$ & Status $^{c}$ & LSU & ITS & SSU & Note \\
\hline Dictyochaeta simplex & GZCC 18-0017 & & MN104620 & MN104609 & MN104629 & \\
\hline Dictyochaeta terminalis & GZCC 18-0085 & $\mathbf{T}$ & MN104624 & MN104613 & MN104633 & \\
\hline Dinemasporium decipiens & CBS 592.73 & & JQ889291 & JQ889275 & - & \\
\hline Dinemasporium morbidum & CBS 129.66 & $\mathrm{~T}$ & JQ889296 & JQ889280 & - & \\
\hline Dinemasporium morbidum & CBS 995.97 & & JQ889297 & JQ889281 & - & \\
\hline Dinemasporium nelloi & MFLUCC 13-0482 & $\mathrm{T}$ & KP711363 & KP711358 & KP711368 & \\
\hline Dinemasporium polygonum & CBS 516.95 & $\mathrm{~T}$ & NG_059109 & NR_137786 & - & \\
\hline Dinemasporium pseudoindicum & CBS 127402 & $\mathrm{~T}$ & JQ889293 & NR_137787 & - & \\
\hline Echinosphaeria canescens & SMH 4791 & & AY436403 & - & - & Helminthosphaeriaceae \\
\hline Ellisembia brachypus & HKUCC 10555 & & DQ408563 & - & - & ?Sporidesmium brachypus \\
\hline Eucalyptostroma eucalypti & CBS 142074 = CPC 28764 & $\mathrm{~T}$ & NG_059257 & NR_154027 & - & \\
\hline Eucalyptostroma eucalypti & CBS $142075=$ CPC 28748 & & KY173499 & KY173407 & - & \\
\hline Eucalyptostroma eucalyptorum & СРС 31800 & $\mathrm{~T}$ & MH327838 & NR_159834 & - & \\
\hline Exserticlava vasiformis & TAMA 450 & & AB753846 & - & - & \\
\hline Gelasinospora tetrasperma & CBS 178.33 = AFTOL-ID 1287 & & DQ470980 & NR_077163 & DQ471032 & Outgroup \\
\hline Gonytrichum caesium & CBS 696.74 & & MH872625 & - & - & \\
\hline $\begin{array}{l}\text { Gonytrichum caesium var. } \\
\text { chloridioides }\end{array}$ & CBS 129.72 & & MH872151 & MH860413 & - & \\
\hline Gonytrichum macrocladum & CBS 201.55 & & MH868989 & MH857448 & - & \\
\hline Gonytrichum macrocladum & CBS 195.60 & & MH869503 & MH857954 & - & \\
\hline Gonytrichum macrocladum & CBS 875.68 & & MH870968 & MH859240 & - & \\
\hline Gonytrichum mirabile & CBS 408.76 & & MH872760 & MH860990 & - & \\
\hline Helminthosphaeria clavariarum & SMH 4609 & $\mathrm{~T}$ & AY346283 & - & - & Helminthosphaeriaceae \\
\hline Infundibulomyces cupulata & BCC 11929 & $\mathrm{~T}$ & EF113979 & EF113976 & EF113982 & \\
\hline Infundibulomyces oblongisporus & BCC 13400 & $\mathrm{~T}$ & EF113980 & EF113977 & EF113983 & \\
\hline Kionochaeta castaneae & GZCC 18-0025 & $\mathbf{T}$ & MN104621 & MN104610 & MN104630 & \\
\hline Kionochaeta ivoriensis & CBS 374.76 = JCM 9876 & $\mathrm{~T}$ & MH872758 & NR_160149 & NG_063387 & \\
\hline Kionochaeta microspora & GZCC 18-0036 & $\mathbf{T}$ & MN104618 & MN104607 & MN104627 & \\
\hline Kionochaeta ramifera & JCM9756 & & - & - & AB003788 & \\
\hline Kionochaeta spissa & JCM9817 & & - & - & AB003789 & \\
\hline Kionochaeta spissa & JCM9818 & & - & - & AB003790 & \\
\hline Lecythothecium duriligni & CBS 101317 & & AF261071 & - & - & \\
\hline
\end{tabular}


Table 1 Continued.

\begin{tabular}{|c|c|c|c|c|c|c|}
\hline Taxa & Isolate $^{a, b}$ & Status $^{c}$ & LSU & ITS & SSU & Note \\
\hline Leptosporella arengae & MFLUCC 15-0330 & $\mathrm{T}$ & MG272246 & MG272255 & MG366594 & Leptosporellaceae \\
\hline Leptosporella gregaria & SMH 4290 & $\mathrm{~T}$ & AY346290 & - & - & Leptosporellaceae \\
\hline Linocarpon arengae & MFLUCC 15-0331 & $\mathrm{T}$ & MG272247 & - & MG366596 & Linocarpaceae \\
\hline Linocarpon cocois & MFLUCC 15-0812 & $\mathrm{T}$ & MG272248 & MG272257 & MG272253 & Linocarpaceae \\
\hline Melanochaeta aotearoae & SMH 1655 & & AF466081 & - & - & \\
\hline Melanochaeta aotearoae & SMH 3551 & & AF466082 & - & - & \\
\hline Melanopsammella gonytrichii & SMH 3785 & & AF466085 & - & - & Now known as Chloridium gonytrichii \\
\hline Melanopsammella vermicularioides & SMH 1985 & & AF064644 & - & - & \\
\hline Melanopsammella vermicularioides & SMH 3883 & & AF466086 & - & - & \\
\hline Melanopsammella vermicularioides & FC 404 & & AF466087 & - & - & $\begin{array}{l}\text { Now known as Chloridium } \\
\text { vermicularioides }\end{array}$ \\
\hline Menispora glauca & FMR 12089 & & HF678538 & HF678528 & - & \\
\hline Menispora tortuosa & CBS 214.56 & & AF178558 & AF178558 & - & \\
\hline Menispora tortuosa & $\begin{array}{l}\text { DAOM } 231154 \text { = AFTOL-ID } \\
278\end{array}$ & & AY544682 & KT225527 & AY544723 & \\
\hline Menisporopsis anisospora & CBS 109475 & $\mathrm{~T}$ & MH874421 & MH862827 & - & \\
\hline Menisporopsis breviseta & GZCC 18-0071 & $\mathbf{T}$ & MN104623 & MN104612 & MN104632 & \\
\hline Menisporopsis dushanensis & GZCC 18-0084 & $\mathbf{T}$ & MN104626 & MN104615 & MN104635 & \\
\hline Menisporopsis pandanicola & KUMCC 17-0271 & $\mathrm{T}$ & МH376726 & MH388353 & МH388320 & \\
\hline Menisporopsis theobromae & MFLUCC 15-0055 & & KX609954 & KX609957 & - & \\
\hline Morrisiella indica & HKUCC 10827 & & DQ408578 & - & - & \\
\hline Multiguttulispora sympodialis & MFLUCC 18-0153 & $\mathbf{T}$ & MN104617 & MN104606 & - & \\
\hline Nawawia filiformis & MFLUCC 16-0853 & & MH758206 & - & MH758200 & \\
\hline Nawawia filiformis & MFLUCC 17-2394 & & MH758209 & MH758196 & MH758203 & \\
\hline Nawawia malaysiana & CPC 16757 = CBS 125544 & $\mathrm{~T}$ & GU229887 & GU229886 & - & $\begin{array}{l}\text { Now known as Neonawawia } \\
\text { malaysiana }\end{array}$ \\
\hline Neopseudolachnella acutispora & HHUF 29727 & $\mathrm{~T}$ & NG_059404 & NR_154223 & - & \\
\hline Neopseudolachnella magnispora & MAFF 244359 & $\mathrm{~T}$ & AB934042 & AB934066 & - & \\
\hline Neopseudolachnella uniseptata & MAFF 244360 & $\mathrm{~T}$ & AB934043 & AB934067 & - & \\
\hline Paliphora intermedia & CBS 896.97 & $\mathrm{~T}$ & NG_057766 & NR_160203 & EF204518 & \\
\hline Paliphora intermedia & CBS 199.95 & & EF204500 & - & EF204517 & \\
\hline Phaeostalagmus cyclosporus & CBS 663.70 & & MH871680 & MH859892 & - & \\
\hline
\end{tabular}


Table 1 Continued.

\begin{tabular}{|c|c|c|c|c|c|c|}
\hline Taxa & Isolate $^{\mathrm{a}, \mathrm{b}}$ & Status $^{\mathrm{c}}$ & LSU & ITS & SSU & Note \\
\hline Phaeostalagmus cyclosporus & CBS 312.75 & & MH872661 & - & - & \\
\hline Phialosporostilbe scutiformis & MFLUCC 17-0227 & $\mathrm{T}$ & MH758207 & MH758194 & MH758201 & \\
\hline Phialosporostilbe scutiformis & MFLUCC 18-1288 & & MH758212 & MH758199 & - & \\
\hline Polynema podocarpi & СРС 32761 & $\mathrm{~T}$ & MH327833 & MH327797 & - & \\
\hline Pseudodinemasporium fabiforme & MAFF 244361 & $\mathrm{~T}$ & AB934044 & AB934068 & - & \\
\hline Pseudolachnea fraxini & CBS 113701 & $\mathrm{~T}$ & JQ889301 & JQ889287 & - & \\
\hline Pseudolachnea hispidula & MAFF 244364 & & AB934047 & AB934071 & - & \\
\hline Pseudolachnella asymmetrica & MAFF 244366 & $\mathrm{~T}$ & AB934049 & AB934073 & - & \\
\hline Pseudolachnella botulispora & MAFF 244367 & $\mathrm{~T}$ & AB934050 & AB934074 & - & \\
\hline Pseudolachnella guaviyunis & CBS 134695 = CMW 39055 & $\mathrm{~T}$ & NG_058879 & NR_153892 & - & $\begin{array}{l}\text { Now known as Calvolachnella } \\
\text { guaviyunis }\end{array}$ \\
\hline Pyrigemmula aurantiaca & CBS 126743 & $\mathrm{~T}$ & HM241692 & HM241692 & - & \\
\hline Pyrigemmula aurantiaca & CBS 126744 & & HM241693 & HM241693 & - & \\
\hline Rattania setulifera & GUFCC 15501 & & HM171322 & GU191794 & - & \\
\hline Ruzenia spermoides & SMH 4606 & & AY436422 & - & - & Helminthosphaeriaceae \\
\hline Sordaria fimicola & CBS 508.50 & & MH868251 & MH856730 & - & Outgroup \\
\hline Sporoschisma hemipsilum & MFLUCC 15-0615 & & KX358074 & KX505869 & - & \\
\hline Sporoschisma hemipsilum & SMH 2125 & & AF466083 & - & - & \\
\hline Sporoschisma hemipsilum & SMH 3251 & & AF466084 & - & - & \\
\hline Sporoschisma longicatenatum & MFLUCC 16-0180 & & KX358077 & KX505871 & - & \\
\hline Sporoschisma mirabile & FMR 11247 & & HF677183 & HF677174 & HF937358 & \\
\hline Sporoschisma palauense & MFLUCC 15-0616 & $\mathrm{T}$ & KX358075 & KX505870 & - & \\
\hline Stanjehughesia vermiculata & HKUCC 10840 & & DQ408570 & - & - & \\
\hline Striatosphaeria codinaeophora & MR 1230 & & AF178546 & AF178546 & - & \\
\hline Striatosphaeria codinaeophora & SMH 1524 & & AF466088 & - & - & \\
\hline Synaptospora plumbea & SMH3962 & & KF765621 & - & - & Helminthosphaeriaceae \\
\hline Tainosphaeria aseptata & GZCC 18-0044 & $\mathbf{T}$ & MN104622 & MN104611 & MN104631 & \\
\hline Tainosphaeria crassiparies & SMH 1934 & $\mathrm{~T}$ & AF466089 & - & - & \\
\hline Tainosphaeria jonesii & GZCC 16-0053 & & KY026056 & KY026059 & - & \\
\hline Tainosphaeria jonesii & GZCC 16-0065 & & KY026057 & KY026060 & - & \\
\hline Tainosphaeria monophialidica & MFLUCC 18-0146 & $\mathbf{T}$ & MN104616 & - & - & \\
\hline Tainosphaeria siamensis & MFLUCC 15-0607 & $\mathrm{T}$ & KX609953 & KX609956 & - & \\
\hline
\end{tabular}


Table 1 Continued.

\begin{tabular}{|c|c|c|c|c|c|c|}
\hline Taxa & Isolate $^{\mathrm{a}, \mathrm{b}}$ & Status $^{\mathrm{c}}$ & LSU & ITS & SSU & Note \\
\hline Thozetella fabacearum & MFLUCC 15-1020 & $\mathrm{T}$ & NG_059767 & KY212754 & - & \\
\hline Thozetella nivea & & & EU825200 & EU825201 & - & \\
\hline Thozetella pandanicola & MFLUCC 16-0253 & $\mathrm{T}$ & MH376740 & МH388366 & - & \\
\hline Thozetella pinicola & RJ-2008 & & EU825195 & EU825197 & EU825198 & \\
\hline Thozetella tocklaiensis & CBS 378.58 & $\mathrm{~T}$ & MH869349 & MH857817 & - & $\begin{array}{l}\text { Before known as Thozetellopsis } \\
\text { tocklaiensis }\end{array}$ \\
\hline Umbrinosphaeria caesariata & CBS 102664 & & AF261069 & - & - & $\begin{array}{l}\text { Now known as Stanjehughesia } \\
\text { hormiscioides }\end{array}$ \\
\hline Verhulstia trisororum & CBS 143234 & $\mathrm{~T}$ & MG022160 & MG022181 & - & \\
\hline Zanclospora iberica & FMR 11584 = CBS 130426 & $\mathrm{~T}$ & KY853544 & KY853480 & HF937360 & \\
\hline Zanclospora iberica & FMR 12186 & & KY853545 & KY853481 & HF937361 & \\
\hline Zanclospora novae-zelandiae & CBS 130280 = FMR 11022 & & MH877214 & - & - & \\
\hline Zignoëlla ovoidea & SMH 2605 & & AF064641 & - & - & Now known as Menispora ovoidea \\
\hline Zignoëlla pulviscula & MUCL 15710 & & AF466090 & - & - & Now known as Menispora pulviscula \\
\hline Zignoëlla pulviscula & SMH 3289 & & AF466091 & - & - & Now known as Menispora pulviscula \\
\hline
\end{tabular}

${ }^{a}$ Newly generated sequences are indicated in bold.

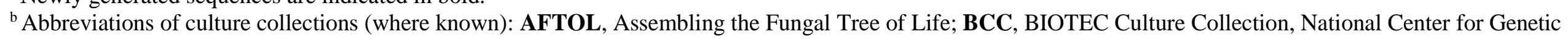

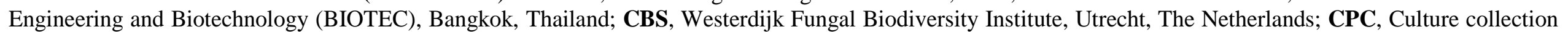

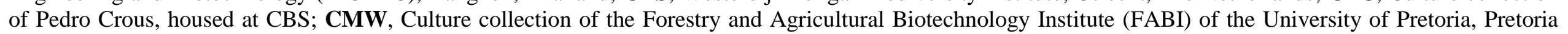

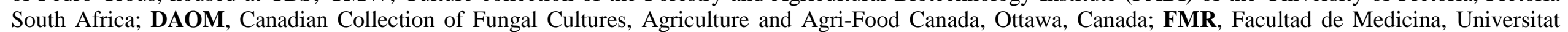

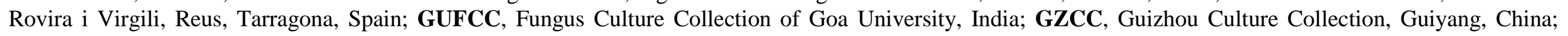

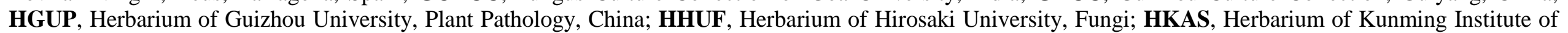

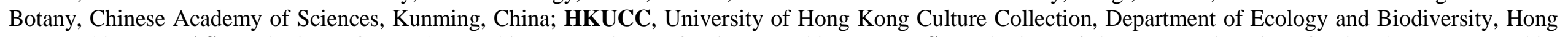

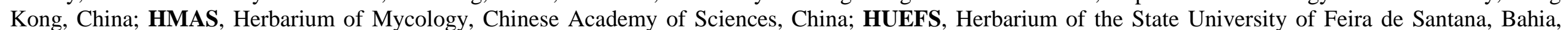

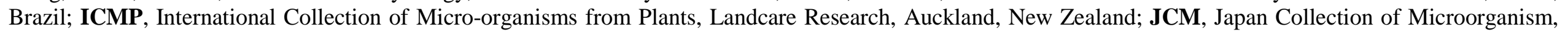

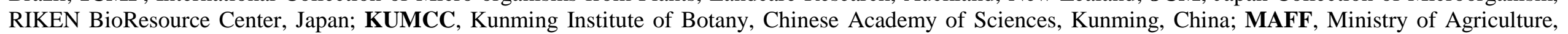

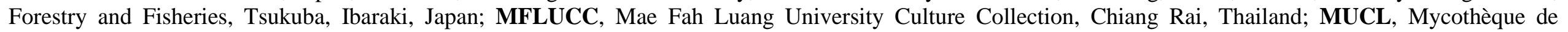

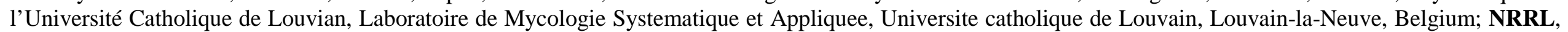

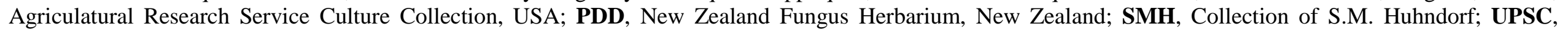
Uppsala University Culture Collection, Uppsala University, Uppsala, Sweden

c Status: T: ex-type; IT: ex-isotype.

${ }^{\mathrm{d}}$ No data in GenBank. 


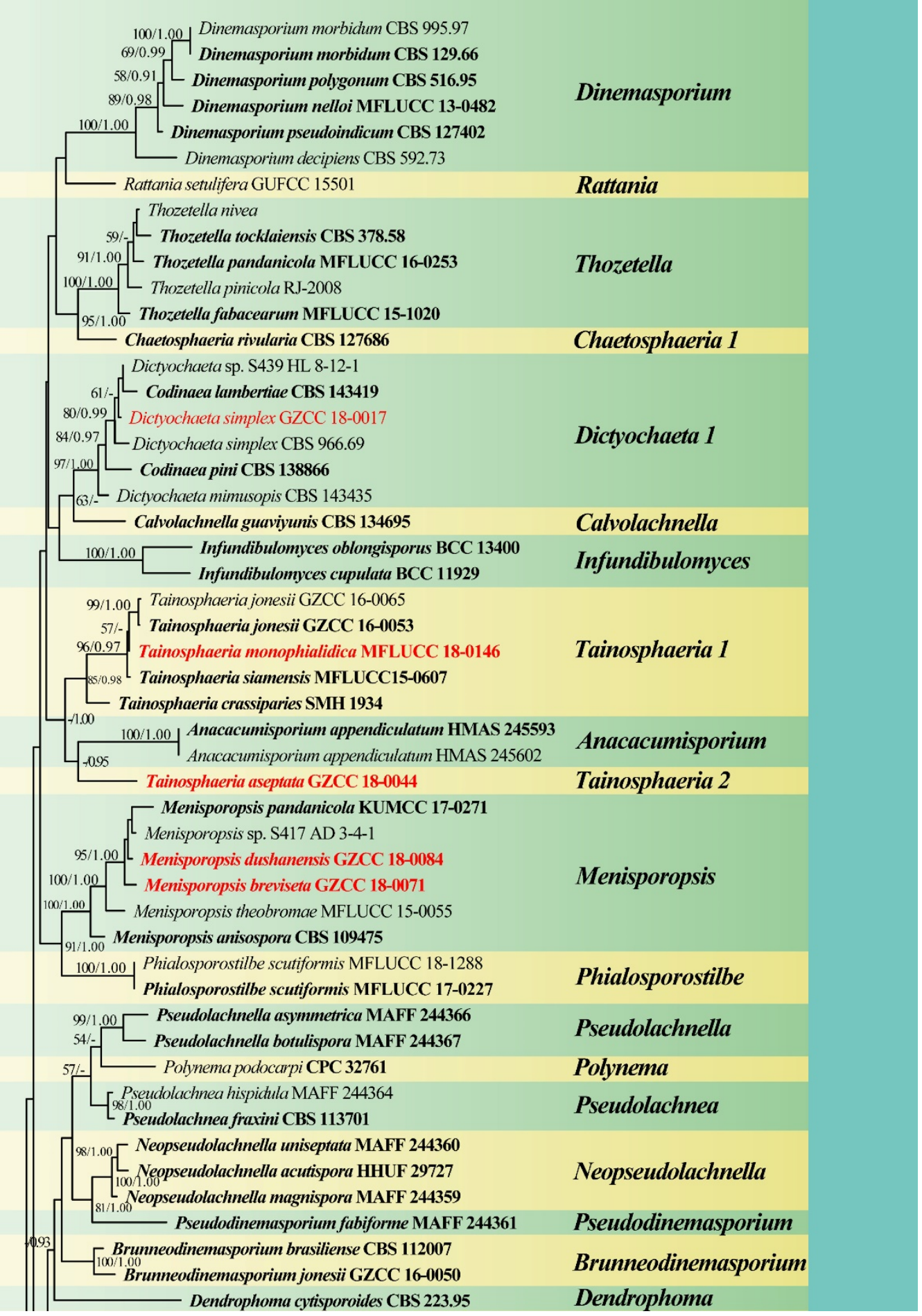

Figure 1 - Phylogenetic tree generated from maximum likelihood (ML) analysis based on combined LSU and ITS sequence data for the family Chaetosphaeriaceae. Bootstrap support values for maximum likelihood greater than $50 \%$ and Bayesian posterior probabilities (PP) greater than 0.8 are indicated above or below the nodes as ML/PP. Ex-type strains are in bold, the new isolates are in red. The tree is rooted with Gelasinospora tetrasperma (AFTOL-ID 1287) and Sordaria fimicola (CBS 508.50). 


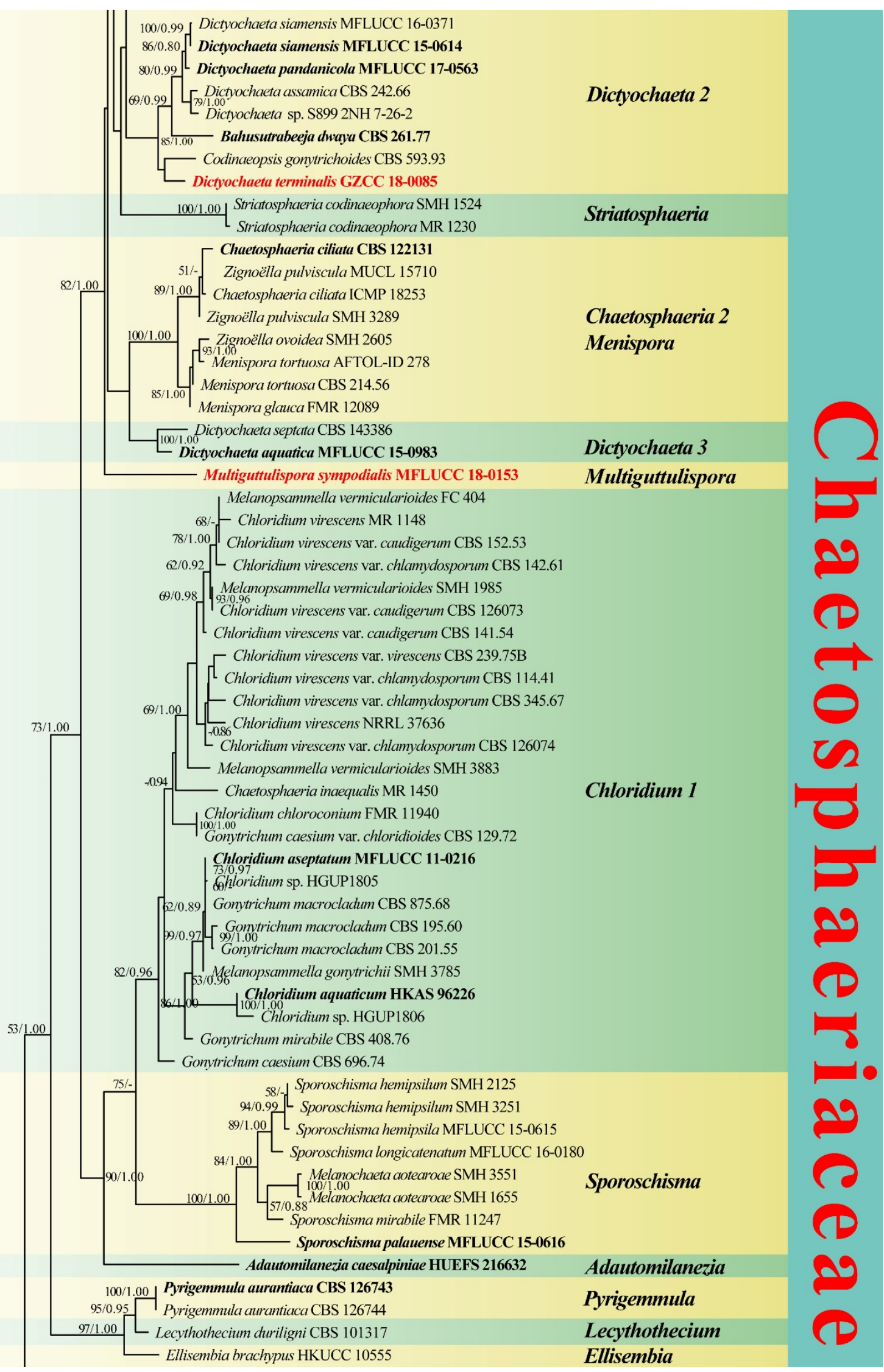

Figure 1 - Continued. 


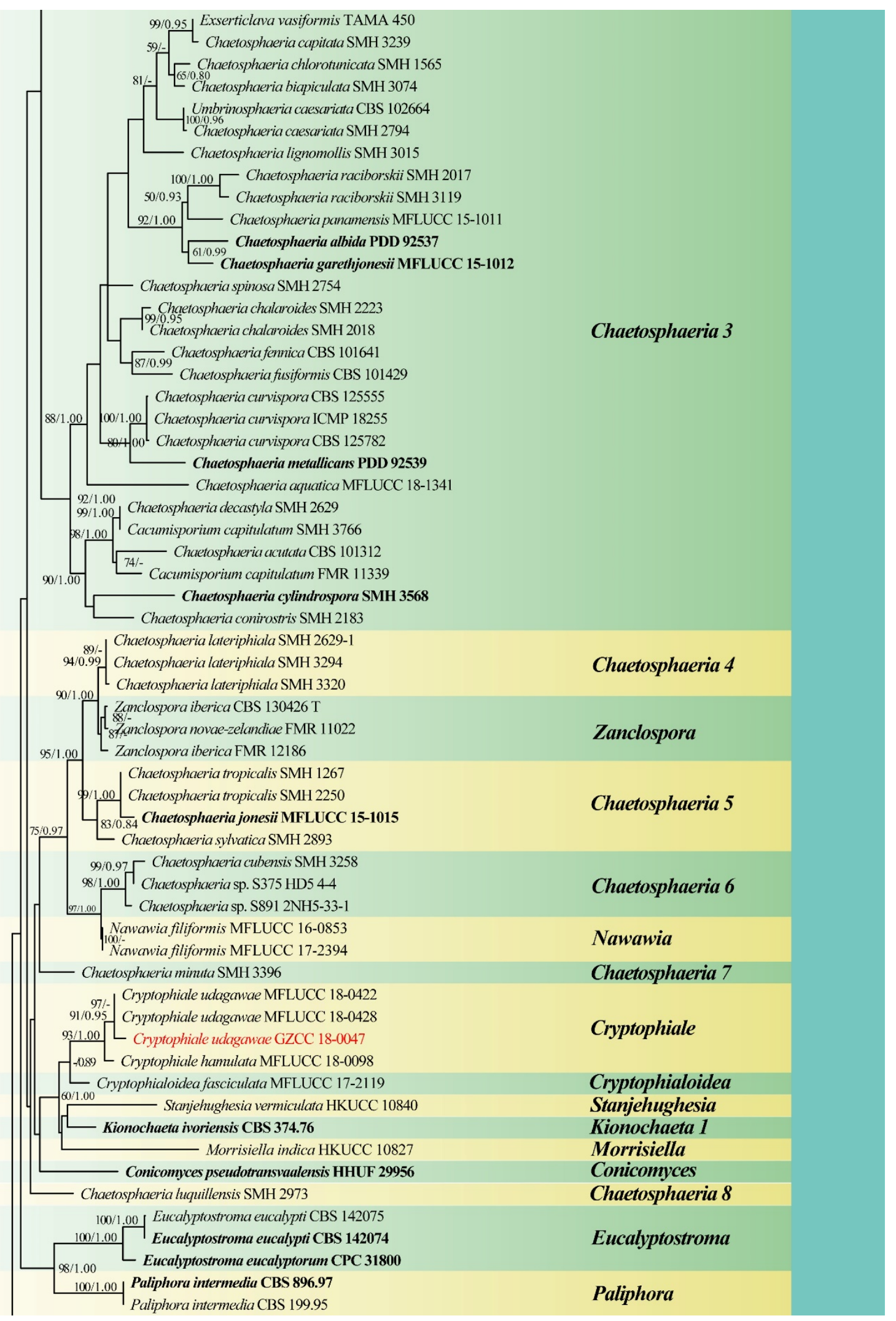

Figure 1 - Continued. 


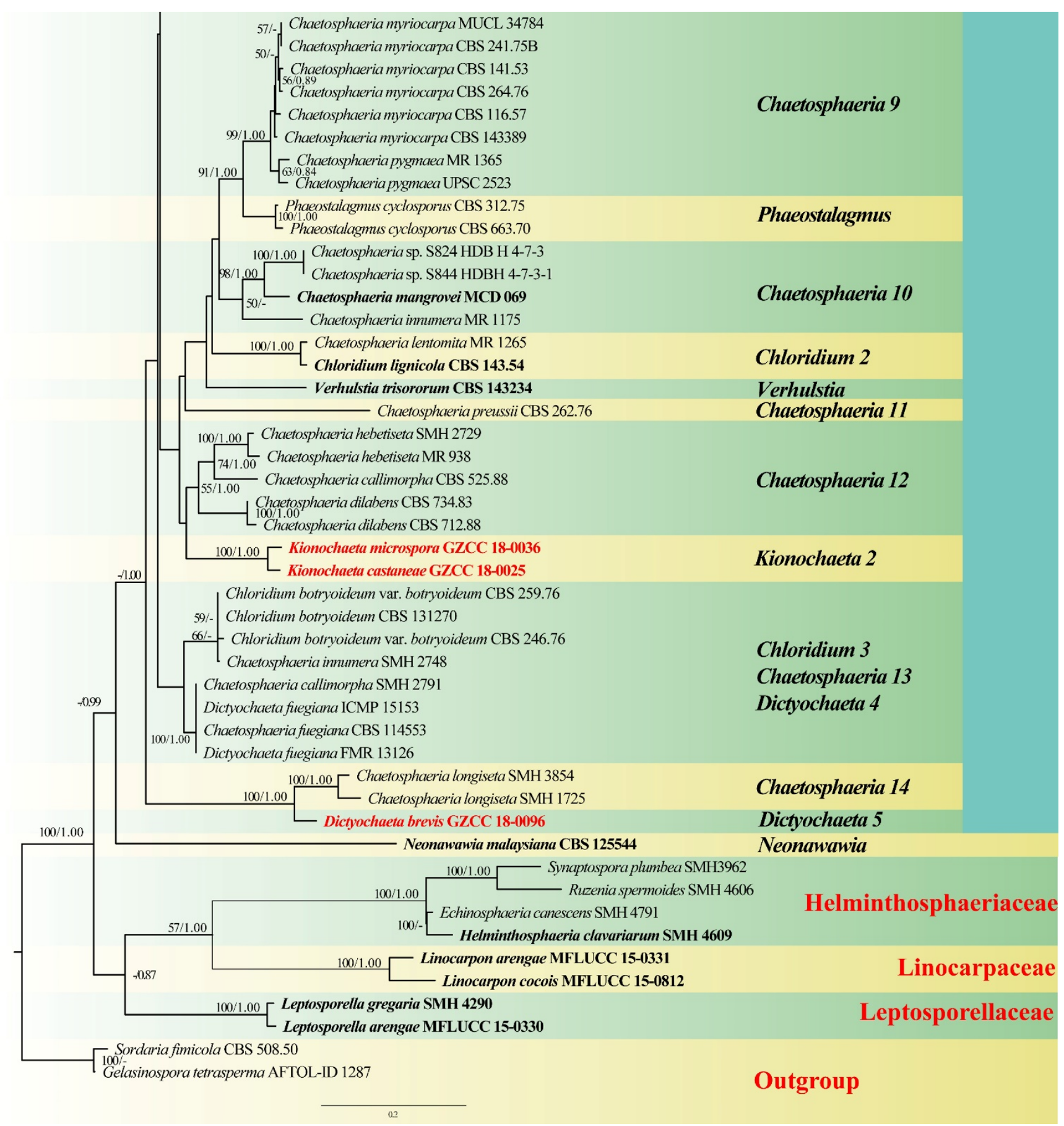

Figure 1 - Continued.

Notes - This is the second report of Cryptophiale udagawae from China and we provide description, sequences data and phylogenetic analysis for this species. This collection fits well with the description of Pirozynski (1968) and with the first description of a Chinese isolate by Yang et al. (2018). The phylogenetic result (Fig. 1) showed that our isolate clustered with C. udagawae (MFLUCC 18-0422 and MFLUCC 18-0428) with high support (91\% MLBS, 0.95 PP). Thus, we identify our collection as C. udagawae.

Dictyochaeta brevis C.G. Lin \& K.D. Hyde, sp. nov.

Fig. 4

Index Fungorum number: IF556705; Facesoffungi number: FoF06285

Etymology - Referring to its short conidiophores.

Holotype - MFLU 19-0216 


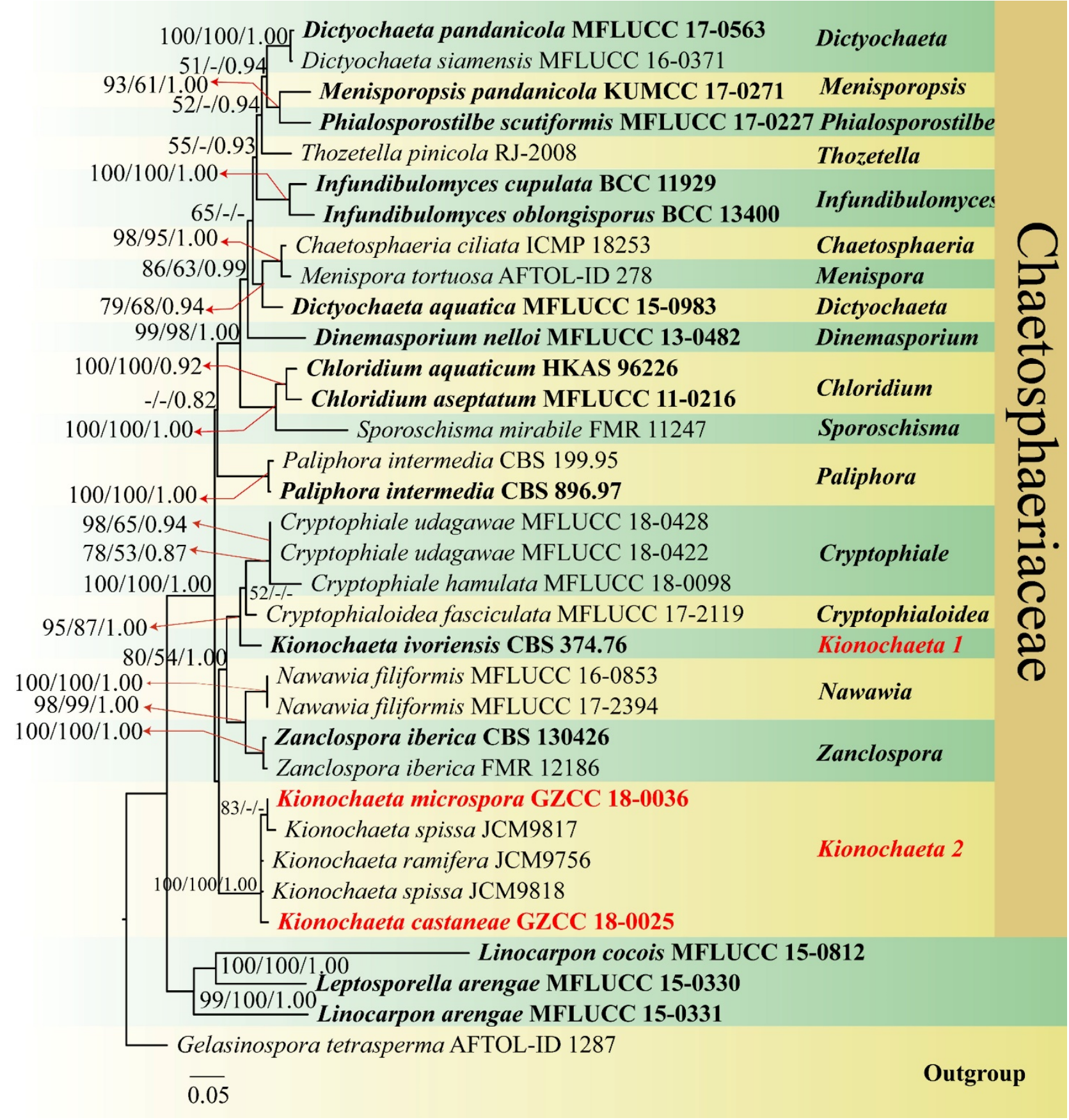

Figure 2 - Phylogenetic tree generated from maximum likelihood (ML) analysis based on combined SSU, ITS and LSU sequence data for selected genera within family Chaetosphaeriaceae. Bootstrap support values for maximum likelihood and maximum parsimony greater than $50 \%$ and Bayesian posterior probabilities (PP) greater than 0.8 are indicated above or below the nodes as $\mathrm{ML} / \mathrm{MP} / \mathrm{PP}$. Ex-type strains are in bold, the new isolates are in red. The tree is rooted with Gelasinospora tetrasperma (AFTOL-ID 1287).

Saprobic on decaying wood. Asexual morph: Colonies on plant substrate effuse, gregarious, white to brown, shining. Mycelium mostly immersed, composed of branched, septate, smooth, thinwalled, pale brown hyphae. Setae absent. Conidiophores mononematous, macronematous, sometimes in groups, pale brown at the base becoming subhyaline or hyaline towards the apex, straight or slightly flexuous, septate, unbranched, smooth, cylindrical, swollen at the base, 13.551(-102) $\mu \mathrm{m}$ long, 2-4 $\mu \mathrm{m}$ wide just above the basal cell, sometimes reduced to conidiogenous cells. Conidiogenous cells monophialidic, with flared collarette, terminal, integrated, cylindrical, 12.7-33.6 × 2.5-3.9 $\mu \mathrm{m}$. Conidia aggregated in large, slimy, white and glistening heads at the apex 
of conidiophores, acrogenous, aseptate, fusiform, most curved, with hair-like and 3.5-6.5 $\mu \mathrm{m}$ long appendages at both ends, 0-2 guttules, smooth, hyaline, 7.5-11.4 $\mu \mathrm{m}(\mathrm{av} .=9.0 \mu \mathrm{m}, \mathrm{n}=45)$ long, 2.0-2.9 $\mu \mathrm{m}$ (av. = $2.5 \mu \mathrm{m}, \mathrm{n}=45)$ wide. Sexual morph: Undetermined.

Material examined - CHINA, Guizhou Province, Qiannan Buyi Miao Autonomous Prefecture, Dushan County, Guizhou Zilinshan National Forest Park (Shengou District), unnamed road, on decaying wood, 6 July 2018, Chuan-Gen Lin, DS 2-39 (MFLU 19-0216, holotype; HKAS 105173, isotype), ex-type living culture GZCC 18-0096.

Notes - Dictyochaeta brevis resembles D. renispora Whitton, McKenzie \& K.D. Hyde in having mononematous conidiophores, monophialidic, terminal conidiogenous cells, hyaline, reniform, aseptate conidia with a setula at each end, and both lacking setae. However, D. brevis differs from $D$. renispora by its shorter and determinate conidiophores, and longer and narrower conidia. Phylogenetically, $D$. brevis grouped in one of the Chaetosphaeria clades but in a separate clade with Chaetosphaeria longiseta F.A. Fernández \& Huhndorf with high support value. Dictyochaeta brevis differs from the asexual morph of $C$. longiseta by its smaller conidia and lacking setae.

Dictyochaeta simplex (S. Hughes \& W.B. Kendr.) Hol.-Jech., Folia geobot. phytotax. 19(4): 434 (1984)

Fig. 5

Facesoffungi number: FoF06286

Saprobic on decaying leaves. Asexual morph: Colonies on plant substrate effuse, gregarious, brown, shining. Mycelium mostly immersed, composed of branched, septate, smooth, thin-walled, brown hyphae. Setae absent Conidiophores mononematous, macronematous, in groups, short, dark brown at the base becoming pale brown towards the apex, straight or slightly flexuous, septate, unbranched, sometimes reduced to conidiogenous cells, smooth, cylindrical, 20-125 $\mu \mathrm{m}$ long, 2.7$5.2 \mu \mathrm{m}$ wide just above the base, which is swollen up to $7.5 \mu \mathrm{m}$. Conidiogenous cells monophialidic or polyphialidic, with flared collarette, terminal, integrated, sometimes sympodial, 8-51 $\mu \mathrm{m}$ long, 3-4 $\mu \mathrm{m}$ wide, slightly narrowing to $1.5-2.3 \mu \mathrm{m}$ just below the collarette. Conidia aggregating in a globose mass at apex of conidiophores, acrogenous, aseptate, long fusiform, curved, with a hair-like, 4.4-7.8 $\mu \mathrm{m}$ long appendage at both ends, hyaline, smooth, 12.7-18.7 $\times$ 2.0-2.9 $\mu \mathrm{m}(\mathrm{av} .=15.6 \times 2.5 \mu \mathrm{m}, \mathrm{n}=30)$ wide. Sexual morph: Undetermined.

Material examined - CHINA, Guizhou Province, Qiannan Buyi Miao Autonomous Prefecture, Dushan County, Guizhou Zilinshan National Forest Park (Shengou District), unnamed road, on decaying leaves, 6 July 2018, Chuan-Gen Lin, DS 1-1 (MFLU 19-0202, HKAS 105104), living culture GZCC 18-0017.

Notes - Our specimen fits well with the description of Dictyochaeta simplex (三 Codinaea simplex) in Hughes \& Kendrick (1968), except they show sympodial proliferation in D. simplex. Our isolate grouped together with $D$. simplex (CBS 966.69) with high support value. Thus, we identify this new specimen as $D$. simplex.

Dictyochaeta terminalis C.G. Lin \& K.D. Hyde, sp. nov.

Fig. 6

Index Fungorum number: IF556706; Facesoffungi number: FoF06287

Etymology - Referring to the terminal conidiogenous cells.

Holotype - MFLU 19-0214

Saprobic on decaying leaves. Asexual morph: Colonies on plant substrate effuse, gregarious, brown, shining. Mycelium mostly immersed, composed of branched, septate, smooth, thin-walled, brown hyphae. Setae fertile, erect, dark brown at the base, paler towards the apex, septate, thickwalled, unbranched, smooth, cylindrical, 150-320 $\mu \mathrm{m}$ long, 3.7-9.4 $\mu \mathrm{m}$ wide at the base. Conidiophores mononematous, macronematous, in groups arising from the mycelial knots from the base of setae, short, sometimes percurrent, dark brown at the base becoming pale brown towards the apex, straight or slightly flexuous, septate, unbranched, smooth cylindrical, 31-171 $\mu \mathrm{m}$ long, 2.7-8.7 $\mu \mathrm{m}$ wide at the base. Conidiogenous cells monophialidic, with flared collarette, terminal, integrated, 13-70 × 3-6 $\mu \mathrm{m}$. Conidia aggregating in a globose mass at apex of conidiophores and 
setae, acrogenous, aseptate, long fusiform, straight to curved, with a hair-like, 4-9.5 $\mu \mathrm{m}$ long appendage at both ends, hyaline, smooth, with 0-2 guttules, 14.7-20.7 $\times 2.9-4.2 \mu \mathrm{m}(\mathrm{av} .=16.9 \times$ $3.6 \mu \mathrm{m}, \mathrm{n}=40)$. Sexual morph: Undetermined.
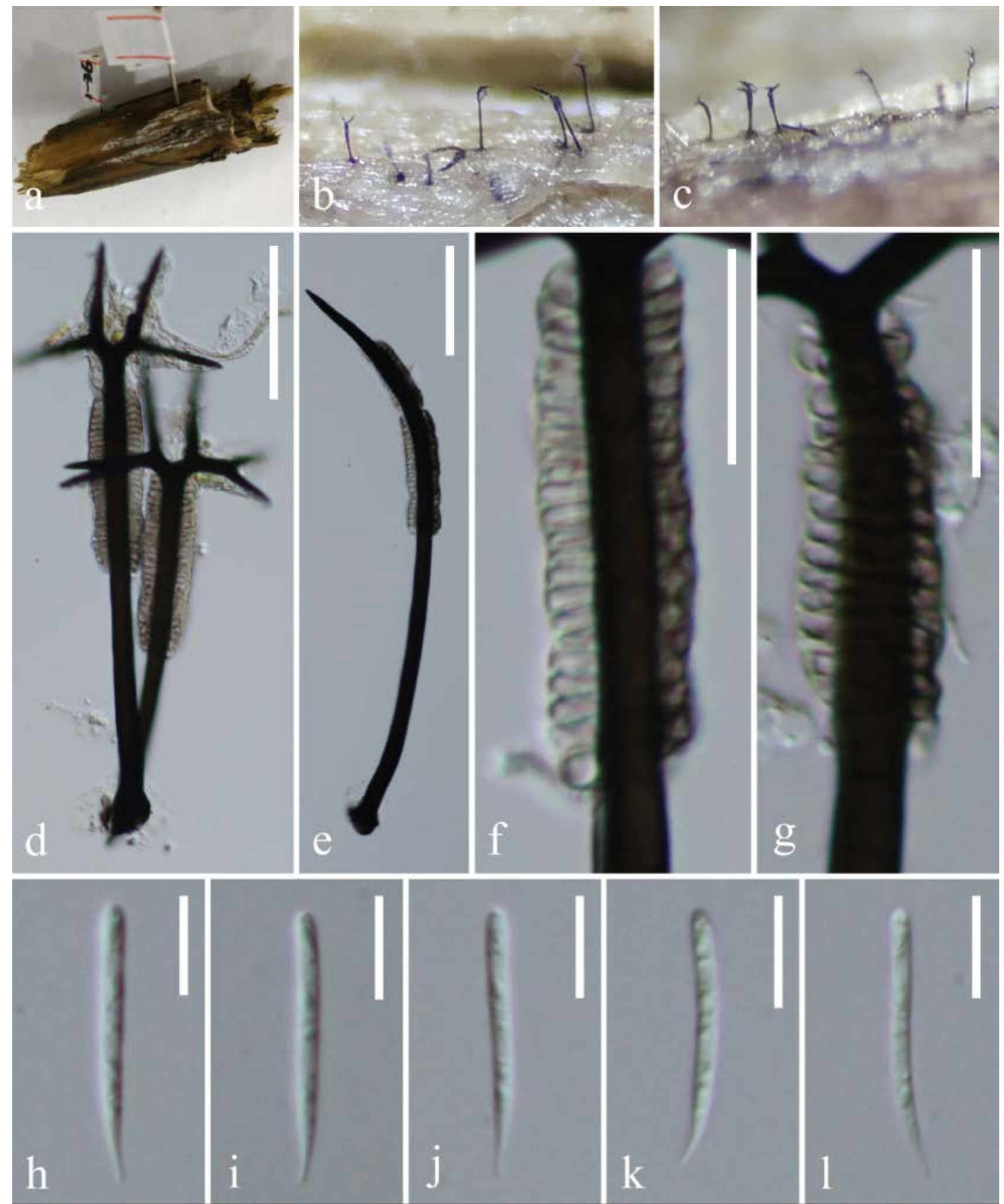

Figure 3 - Cryptophiale udagawae (MFLU 19-0209). a Host material. b, c Conidiophores on the host surface. d, e Conidiophores and conidiogenous cells. f, g Conidiogenous cells. h-l Conidia. Scale bars: $\mathrm{d}, \mathrm{e}=50 \mu \mathrm{m}, \mathrm{f}, \mathrm{g}=20 \mu \mathrm{m}, \mathrm{h}-1=10 \mu \mathrm{m}$. 


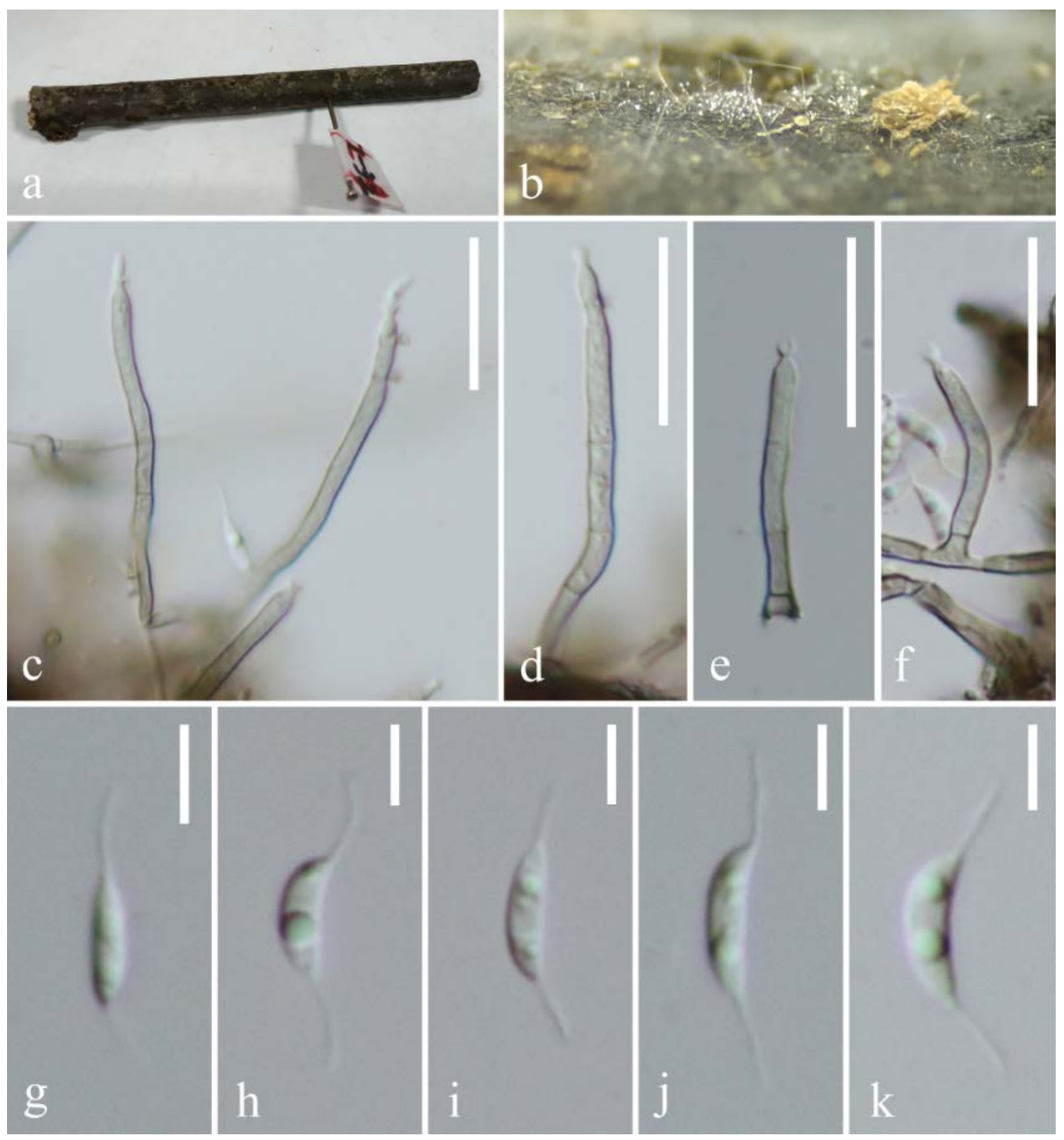

Figure 4 - Dictyochaeta brevis (MFLU 19-0216, holotype). a Host material. b Conidiophores on the host surface. $\mathrm{c}-\mathrm{f}$ Conidiophores and conidiogenous cells. $\mathrm{g}-\mathrm{k}$ Conidia. - Scale bars: $\mathrm{c}-\mathrm{f}=20$ $\mu \mathrm{m}, \mathrm{g}-\mathrm{k}=5 \mu \mathrm{m}$.

Material examined - CHINA, Guizhou Province, Qiannan Buyi Miao Autonomous Prefecture, Dushan County, Guizhou Zilinshan National Forest Park (Shengou District), unnamed road, on decaying leaves, 6 July 2018, Chuan-Gen Lin, DS 2-26 (MFLU 19-0214, holotype; HKAS 105163, isotype), ex-type living culture GZCC 18-0085.

Notes - Dictyochaeta terminalis is similar to Codinaea pakhalensis S.M. Reddy \& S.S. Reddy, D. assamica (Agnihothr.) Aramb., Cabello \& Mengasc., D. fertilis (S. Hughes \& W.B. Kendr.) Hol.-Jech., D. gamundiae Aramb. \& Cabello and D. plovercovensis Goh \& K.D. Hyde in having fertile setae, mononematous conidiophores, and aseptate and hyaline conidia with setulae at each end (Agnihothrudu 1962, Hughes \& Kendrick 1968, Reddy \& Reddy 1977, Arambarri et al. 1987, Kuthubutheen \& Nawawi 1991b, Goh \& Hyde 1999, Whitton et al. 2000). However, 
Dictyochaeta terminalis differs from these species by its conidiogenous cells on setae, the conidiophores are monophialidic and without intercalary conidiogenous loci, and its conidia are bigger. In the phylogeny (Fig. 1), D. terminalis formed a separate clade, and grouped together with Codinaeopsis gonytrichoides (Shearer \& J.L. Crane) Morgan-Jones (CBS 593.93). Codinaeopsis gonytrichoides differs from $D$. terminalis by conidiophores that are setiform, conidiogenous cells are polyphialidic and intercalary and conidia are allantoid, 14-17 $\times 2.5-3 \mu \mathrm{m}$, with a filiform, 6-10 $\mu \mathrm{m}$ long appendage at each end.
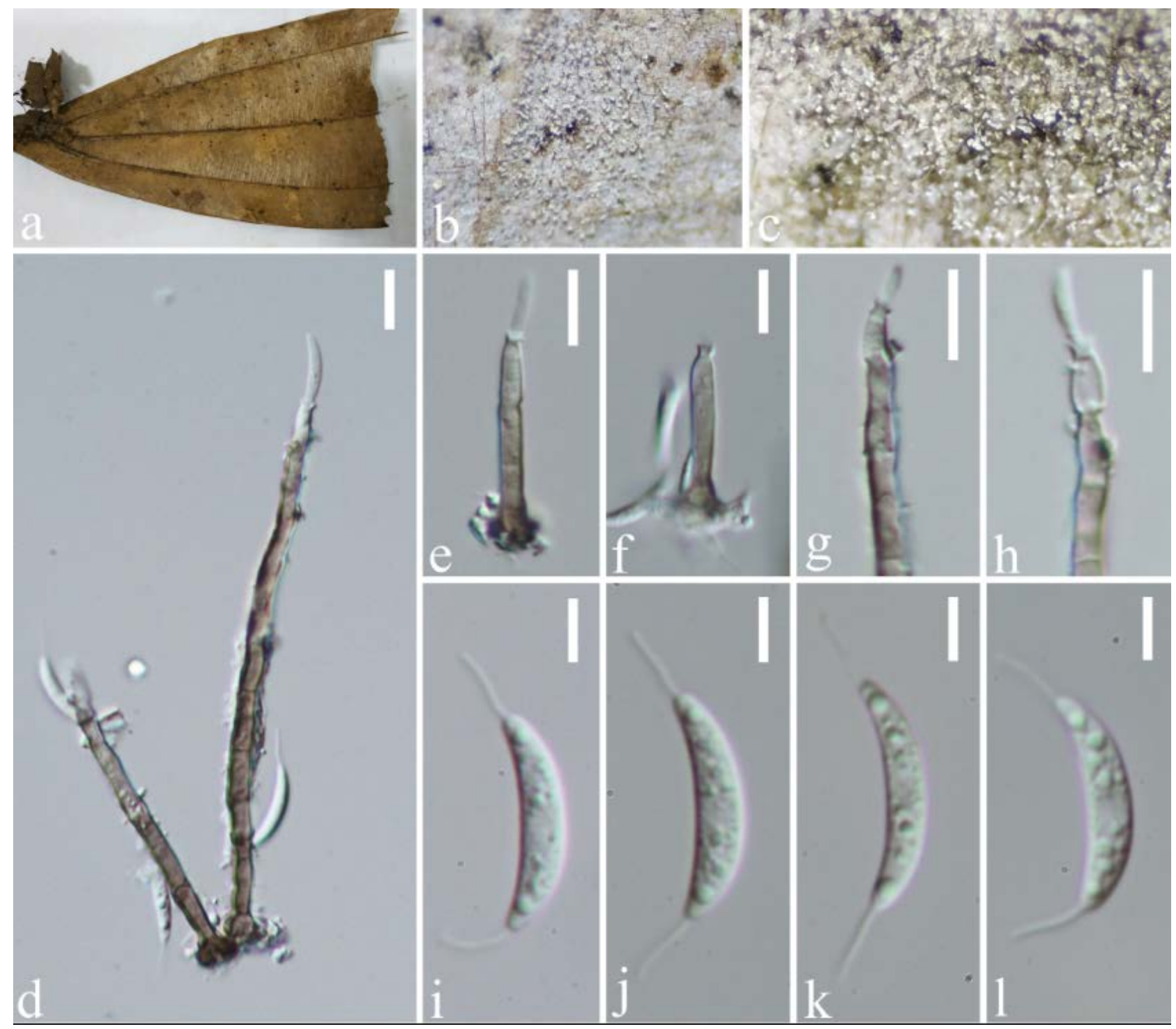

Figure 5 - Dictyochaeta simplex (MFLU 19-0202). a Host material. b, c Conidiophores on the host surface. d-f Conidiophores and conidiogenous cells. g, h Conidiogenous cells. i-l Conidia. Scale bars: $\mathrm{d}-\mathrm{h}=10 \mu \mathrm{m}, \mathrm{i}-\mathrm{l}=5 \mu \mathrm{m}$.

Kionochaeta castaneae C.G. Lin \& J.K. Liu, sp. nov.

Fig. 7

Index Fungorum number: IF556707; Facesoffungi number: FoF06288

Etymology - Referring to the host genus Castanea on which the type specimen was collected. Holotype - MFLU 19-0204

Saprobic on decaying shell of Castanea mollissima. Asexual morph: Colonies on plant substrate effuse, caespitose, glistening, with white and slimy mass of conidia. Mycelium mostly immersed, partially superficial. Conidiophores macronematous, mononematous, setiform, caespitose, erect, straight or slightly curved, dark brown, smooth, thick-walled, sometimes branched above the fertile region, sometimes with secondly fertile region at the apex of the main 
axis, 265-320 $\mu \mathrm{m}$ long, 7-9.5 $\mu \mathrm{m}$ wide at the base; when branched, terminal branches dark brown, 45-105 $\mu \mathrm{m}$ long, 2.5-5.5 $\mu \mathrm{m}$ wide at the base, acutely pointed above. Fertile region immediately below the sterile branches at the middle part of the conidiophores, rarely with a secondly fertile region at the apex of the main axis, consisting of a mass of short branches on which conidiogenous cells are borne. Conidiogenous cells monophialidic, discrete, determinate, intercalary, rarely terminal, narrowly ellipsoid, pale brown, 5.2-7.9 $\times 2-2.8 \mu \mathrm{m}$. Conidia produced in slime and adhering to the fertile region, hyaline, smooth, lunate, cylindrical, aseptate, 5.1-6.5 $\mu \mathrm{m}$ long (av. $=$ $5.9 \mu \mathrm{m}, \mathrm{n}=35), 1.2-1.8 \mu \mathrm{m}$ wide (av. $=1.5 \mu \mathrm{m}, \mathrm{n}=35)$. Sexual morph: Undetermined.

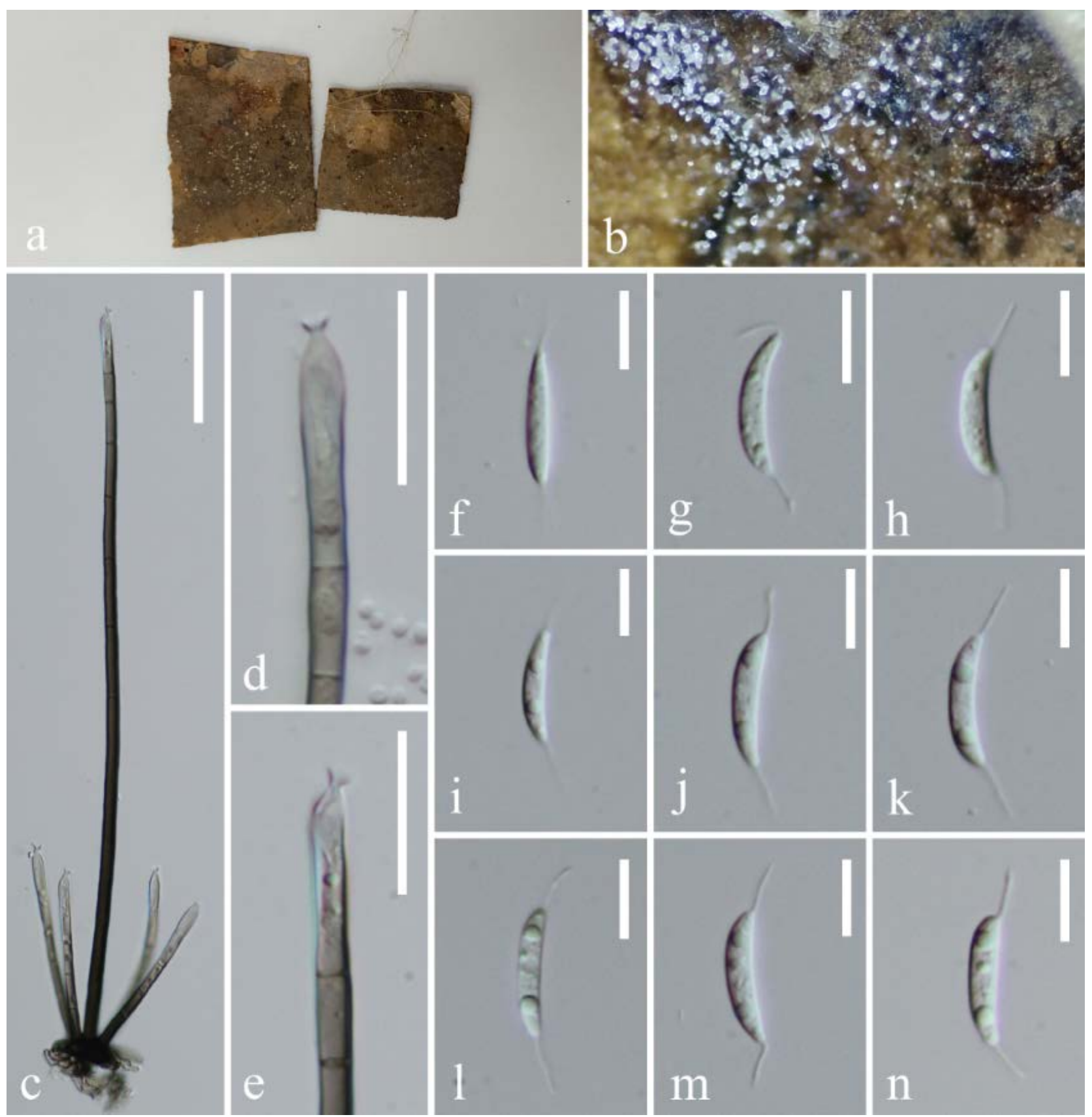

Figure 6 - Dictyochaeta terminalis (MFLU 19-0214, holotype). a Host material. b Conidiophores on the host surface. c Conidiophores and conidiogenous cells. d, e Conidiogenous cells. $\mathrm{f}-\mathrm{n}$ Conidia. Scale bars: $\mathrm{c}=50 \mu \mathrm{m}, \mathrm{d}, \mathrm{e}=20 \mu \mathrm{m}, \mathrm{f}-\mathrm{n}=10 \mu \mathrm{m}$.

Material examined - CHINA, Guizhou Province, Qiannan Buyi Miao Autonomous Prefecture, Dushan County, Guizhou Zilinshan National Forest Park (Shengou District), unnamed road, on decaying shell of Castanea mollissima Blume (Chinese chestnut), 6 July 2018, Chuan-Gen 
Lin, DS 1-10 (MFLU 19-0204, holotype; HKAS 105111, isotype), ex-type living culture GZCC 18-0025.
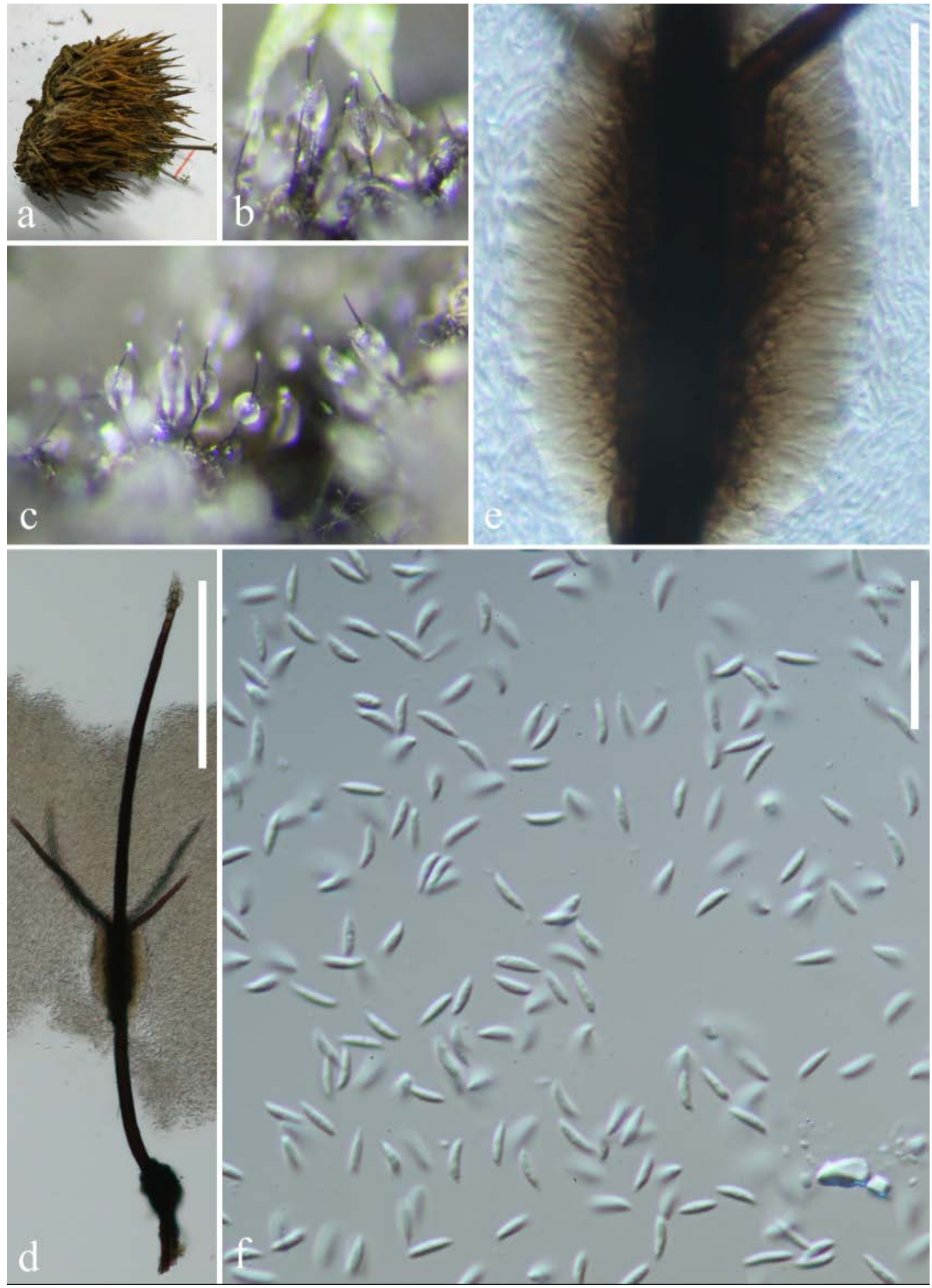

Figure 7 - Kionochaeta castaneae (MFLU 19-0204, holotype). a Host material. b, c Conidiophores on the host surface. d Conidiophore, conidiogenous cells and conidia. e Conidiogenous cells. f Conidia. Scale bars: $d=100 \mu \mathrm{m}$, e, $\mathrm{f}=20 \mu \mathrm{m}$. 
Notes - This is the fifth Kionochaeta species that has accessory lateral setiform branches. The other four are K. australiensis Goh \& K.D. Hyde, K. keniensis (P.M. Kirk) P.M. Kirk \& B. Sutton, K. pughii Kuthub. \& Nawawi and K. ramifera (Kirk \& Sutton 1985, Kuthubutheen \& Nawawi 1988, Goh \& Hyde 1997). Kionochaeta castaneae differs from K. keniensis by its asetulate conidia and from $K$. australiensis and $K$. pughii by its accessory lateral branches being sterile. The conidia of $K$. castaneae are smaller than K. ramifera (5.1-6.5 × 1.2-1.8 $\mu \mathrm{m}$ vs. (5-) 8-12 × 1-1.5(-1.8) $\mu \mathrm{m})$. Phylogenetically, $K$. castaneae shows close relationship with $K$. microspora as they formed a monophyletic group (Fig. 1). However, Kionochaeta castaneae can be recognized as a phylogenetically distinct species. A comparison of ITS sequences data between $K$. castaneae and K. microspora showed that there are 20 bp (base pair) of 505 base pairs differences (including the gaps).

Kionochaeta microspora C.G. Lin \& K.D. Hyde, sp. nov.

Fig. 8

Index Fungorum number: IF556708; Facesoffungi number: FoF06289

Etymology - Referring to its small conidia.

Holotype - MFLU 19-0206

Saprobic on decaying wood. Asexual morph: Colonies on plant substrate effuse, caespitose, glistening, with olive-green to dark green and slimy mass of conidia. Mycelium mostly immersed, partially superficial. Conidiophores macronematous, mononematous, caespitose, erect, straight or slightly curved, setiform, smooth, thick-walled, unbranched, septate, dark brown at the base, paler towards the apex, cylindrical, swollen at the base, 40-305 $\mu \mathrm{m}$ long, 3.3-10.5 $\mu \mathrm{m}$ wide at the base. Fertile region at the apex of the conidiophores, rarely at the middle of the conidiophores with a secondary fertile region at the conidiophore apex, consisting of a mass of short branches on which conidiogenous cells are borne, $12-38 \times 8-23 \mu \mathrm{m}$. Conidiogenous cells monophialidic, discrete, determinate, terminal, rarely intercalary, narrowly ellipsoid, ampulliform, subhyaline to pale brown, 4.5-8.1 $\times 2.5-3.9 \mu \mathrm{m}$. Conidia produced in slime and adhering to the fertile region, hyaline, smooth, lunate, cylindrical or clavate, aseptate, 4.2-6.4 × 1.5-2.3 $\mu \mathrm{m}(\mathrm{av} .=5.5 \times 1.9 \mu \mathrm{m}, \mathrm{n}=40)$. Sexual morph: Undetermined.

Material examined - CHINA, Guizhou Province, Qiannan Buyi Miao Autonomous Prefecture, Dushan County, Guizhou Zilinshan National Forest Park (Shengou District), unnamed road, on decaying wood, 6 July 2018, Chuan-Gen Lin, DS 1-22 (MFLU 19-0206, holotype; HKAS 105121, isotype), ex-type living culture GZCC 18-0036.

Notes - Kionochaeta microspora is similar to K. filamentosa Yanna \& K.D. Hyde, K. nanophora Kuthub. \& Nawawi and K. pleomorpha R.F. Castañeda, W.B. Kendr. \& Guarro by having a fertile region at the apex of the setiform conidiophores without accessory lateral branches and aseptate conidia (Kuthubutheen \& Nawawi 1988, Subramanian 1993, Castañeda Ruíz et al. 1998, Yanna \& Hyde 2002). Kionochaeta filamentosa is a unique species in having hyaline filaments at the conidiophore apex (Yanna \& Hyde 2002). Kionochaeta nanophora and $K$. pleomorpha have a setiform element devoid of a compactly branched conidiogenous apparatus throughout its height, which separates them from other Kionochaeta species (Kuthubutheen \& Nawawi 1988, Subramanian 1993).

Menisporopsis breviseta C.G. Lin \& K.D. Hyde, sp. nov.

Fig. 9

Index Fungorum number: IF556709; Facesoffungi number: FoF06290

Etymology - Referring to the short setae.

Holotype - MFLU 19-0212

Saprobic on decaying leaves. Asexual morph: Colonies on plant substrate effuse, scattered, white to pale brown. Mycelium partly immersed, composed of brown hyphae. Setae central, solitary, erect, straight, unbranched, dark brown, septate, smooth, thick-walled, subulate, swollen at the apex, the lower part of each seta encased by tightly compacted conidiophores, 95-190 $\mu \mathrm{m}$ long, 2.7-5.4 $\mu \mathrm{m}$ thick at the base. Synnemata 52-113 $\mu \mathrm{m}$ long, 6.5-12 $\mu \mathrm{m}$ wide. Conidiophores macronematous, synnematous, encasing lower part of setae; separate filaments unbranched, straight or slightly 
flexuous, lower part narrow, upper part wider, pale brown, smooth, up to $182 \mu \mathrm{m}$ long, upper part 2.5-4.6 $\mu \mathrm{m}$ thick. Conidiogenous cells monophialidic, integrated, terminal, cylindrical, with collarettes. Conidia aggregated in slimy heads, acrogenous, semi-endogenous, appendiculate with one or two setulae at each end, setulae 4-9 $\mu \mathrm{m}$ long, aseptate, curved, cylindrical or fusiform, hyaline, smooth, 14.2-24.3 $\mu \mathrm{m}(\mathrm{av} .=18.8 \mu \mathrm{m}, \mathrm{n}=50)$ long, 2.4-3.6 $\mu \mathrm{m}(\mathrm{av} .=3.1 \mu \mathrm{m}, \mathrm{n}=50)$ wide. Sexual morph: Undetermined.
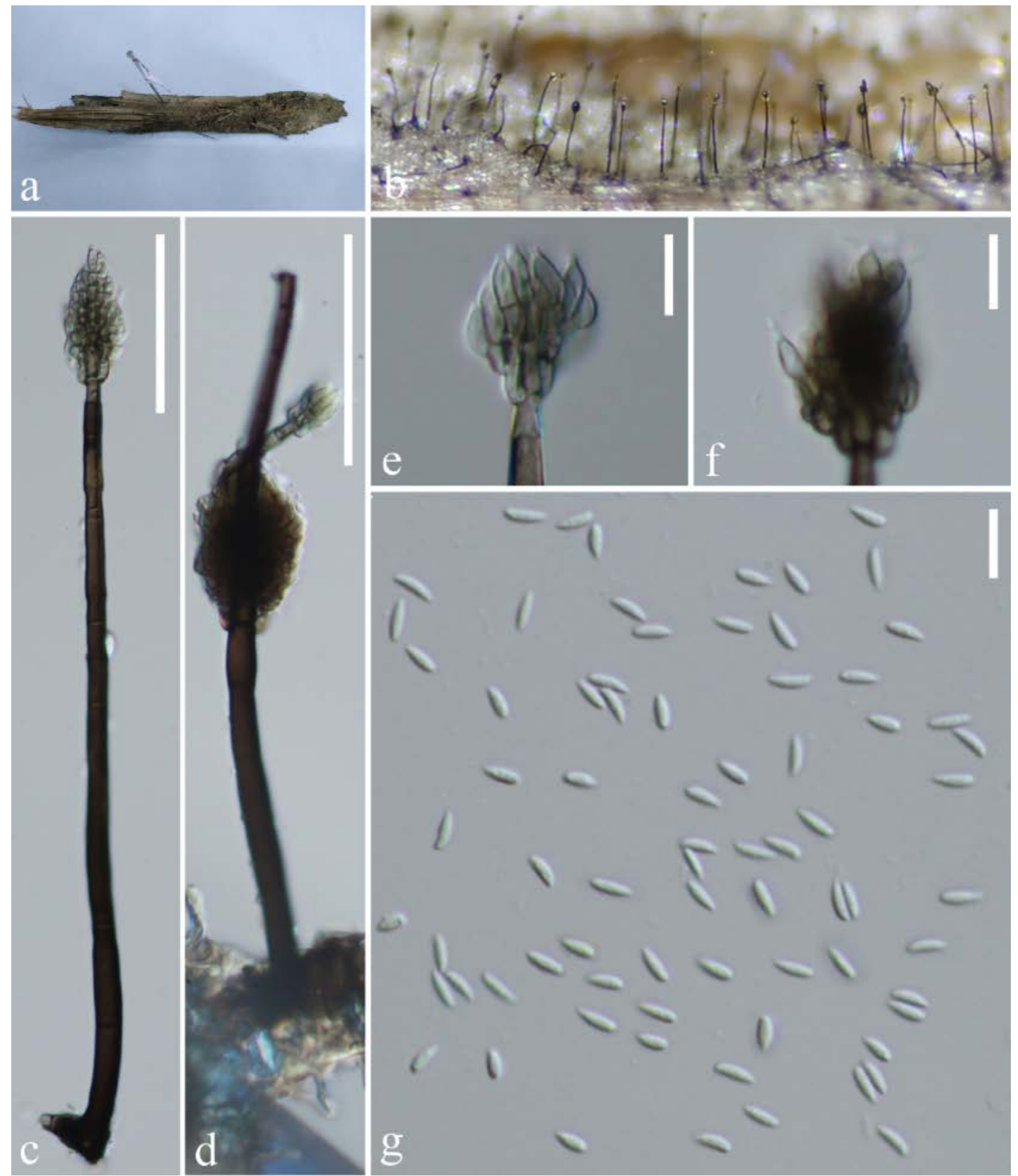

Figure 8 - Kionochaeta microspora (MFLU 19-0206, holotype) a Host material. b Conidiophores on the host surface. c, d Conidiophores and conidiogenous cells. e, f Conidiogenous cells. g Conidia. Scale bars: $\mathrm{c}, \mathrm{d}=50 \mu \mathrm{m}$, e-g $=10 \mu \mathrm{m}$. 

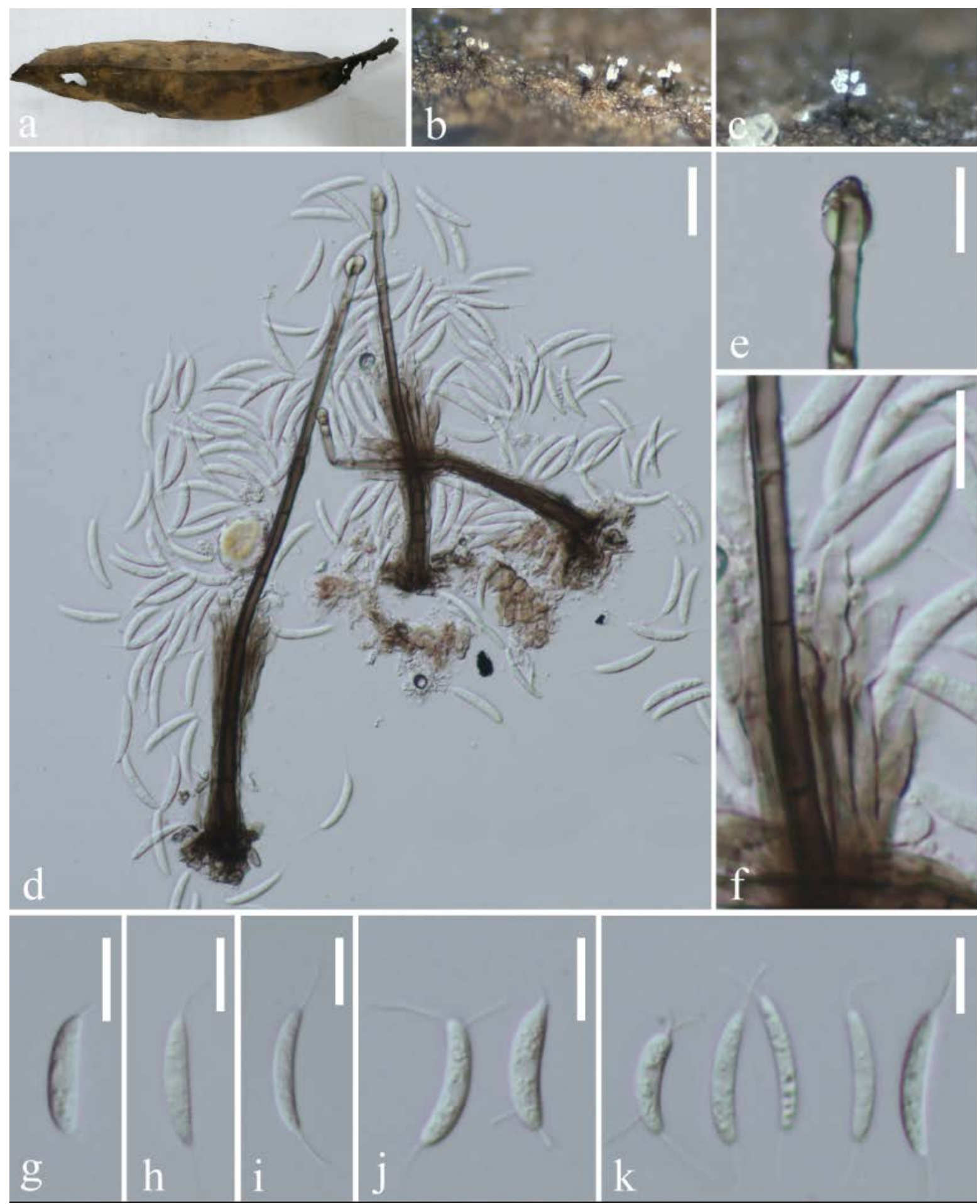

Figure 9 - Menisporopsis breviseta (MFLU 19-0212, holotype) a Host material. b, c Conidiophores on the host surface. d Conidiophores, setae, conidiogenous cells and conidia. e Apex of seta. $\mathrm{f}$ Conidiogenous cells. g-k Conidia. Scale bars: $\mathrm{d}=20 \mu \mathrm{m}$, $\mathrm{e}-\mathrm{k}=10 \mu \mathrm{m}$.

Material examined - CHINA, Guizhou Province, Qiannan Buyi Miao Autonomous Prefecture, Dushan County, Guizhou Zilinshan National Forest Park (Shengou District), unnamed road, on decaying leaves, 6 July 2018, Chuan-Gen Lin, DS 1-76 (MFLU 19-0212, holotype; HKAS 105151, isotype), ex-type living culture GZCC 18-0071.

Notes - The phylogenetic results showed that our isolate clustered with other Menisporopsis species and formed a sister group with M. pandanicola Tibpromma \& K.D. Hyde (Fig. 1). Menisporopsis breviseta is similar to $M$. pandanicola by having lunate, hyaline conidia with 1-2 setula at each end. However, $M$. breviseta differs from $M$. pandanicola by its short setae and 
smaller synnemata. Menisporopsis breviseta can be distinguished from other Menisporopsis species by its short setae and conidia with 1-2 setula at each end. A comparison of ITS sequences data between $M$. breviseta and $M$. pandanicola showed that there are 23 bp (base pair) of 477 base pairs differences (including the gaps).

Menisporopsis dushanensis C.G. Lin \& K.D. Hyde, sp. nov.

Index Fungorum number: IF556710; Facesoffungi number: FoF06291

Etymology - Referring to Dushan, the type locality of this species.

Holotype - MFLU 19-0213

Saprobic on decaying leaves. Asexual morph: Colonies on plant substrate effuse, scattered, white to pale brown. Mycelium partly immersed, composed of brown hyphae. Setae central, solitary, erect, straight, unbranched, dark brown, septate, smooth, thick-walled, subulate, the lower part of each seta encased by tightly compacted conidiophores, 207-455 $\mu \mathrm{m}$ long, 5-10.5 $\mu \mathrm{m}$ wide at the base. Synemata 75-150 $\mu \mathrm{m}$ long, 8.5-22.5 $\mu \mathrm{m}$ wide. Conidiophores macronematous, synnematous, encasing lower part of setae; separate filaments unbranched, straight or slightly flexuous, lower part narrow, upper part wider, pale brown, smooth, up to $147 \mu \mathrm{m}$ long, upper part 2.5-6 $\mu \mathrm{m}$ thick. Conidiogenous cells monophialidic, integrated, terminal, cylindrical, with collarettes. Conidia aggregated in slimy heads, acrogenous, semi-endogenous, appendiculate with one or two setulae at each end, setulae 3-11 $\mu \mathrm{m}$ long, aseptate, curved, cylindrical or fusiform, hyaline, smooth, 14.0-21 $\mu \mathrm{m}(\mathrm{av} .=18 \mu \mathrm{m}, \mathrm{n}=50)$ long, $3-4 \mu \mathrm{m}(\mathrm{av} .=3.5 \mu \mathrm{m}, \mathrm{n}=50)$ wide. Sexual morph: Undetermined.

Material examined - CHINA, Guizhou Province, Qiannan Buyi Miao Autonomous Prefecture, Dushan County, Guizhou Zilinshan National Forest Park (Shengou District), unnamed road, on decaying leaves, 6 July 2018, Chuan-Gen Lin, DS 2-25 (MFLU 19-0213, holotype; HKAS 105162, isotype), ex-type living culture GZCC 18-0084.

Notes - Menisporopsis dushanensis is closely related to other Menisporopsis species which constitute the strongly supported monophyletic Menisporopsis clade. In particular, M. dushanensis is nested in between Menisporopsis sp. (S417 AD 3-4-1) and M. breviseta but can be recognized as a distinct lineage (Fig. 1). The most similar species to $M$. dushanensis are $M$. breviseta and $M$. pandanicola in having lunate, hyaline conidia with 1-2 setula at each end. However, $M$. dushanensis differs from $M$. breviseta by its longer setae, bigger synnemata and wider conidia. Menisporopsis dushanensis differs from $M$. pandanicola by longer setae and wider conidia. A comparison of ITS sequences data between $M$. dushanensis and $M$. pandanicola showed that there are 23 bp (base pair) difference within the 478 base pairs (including the gaps). Therefore, we introduce it as a new species $M$. dushanensis.

Multiguttulispora C.G. Lin \& J.K. Liu gen. nov.

Index Fungorum number: IF556712; Facesoffungi number: FoF06293

Etymology - Referring to the conidia with 0-4 guttules.

Type species - Multiguttulispora sympodialis

Saprobic on decaying plant. Asexual morph: Colonies on plant substrate effuse, scattered, white to pale brown. Mycelium partly immersed, composed of brown hyphae. Conidiophores mononematous, macronematous, erect, straight or flexuous, unbranched, septate, smooth, dark brown at the base becoming light brown towards the apex, cylindrical. Conidiogenous cells polyblastic, integrated, terminal, sympodial, pale brown, cylindrical. Conidia aggregated in slimy mass at the apex of the conidiophores, acrogenous, smooth, hyaline, septate, with guttules, cylindrical, oblong with an appendage at each end. Sexual morph: Undetermined.

Multiguttulispora sympodialis C.G. Lin \& J.K. Liu, sp. nov. Fig. 11

Index Fungorum number: IF556711; Facesoffungi number: FoF06292

Etymology - Referring to the sympodial conidiophores.

Holotype - MFLU 19-0218 

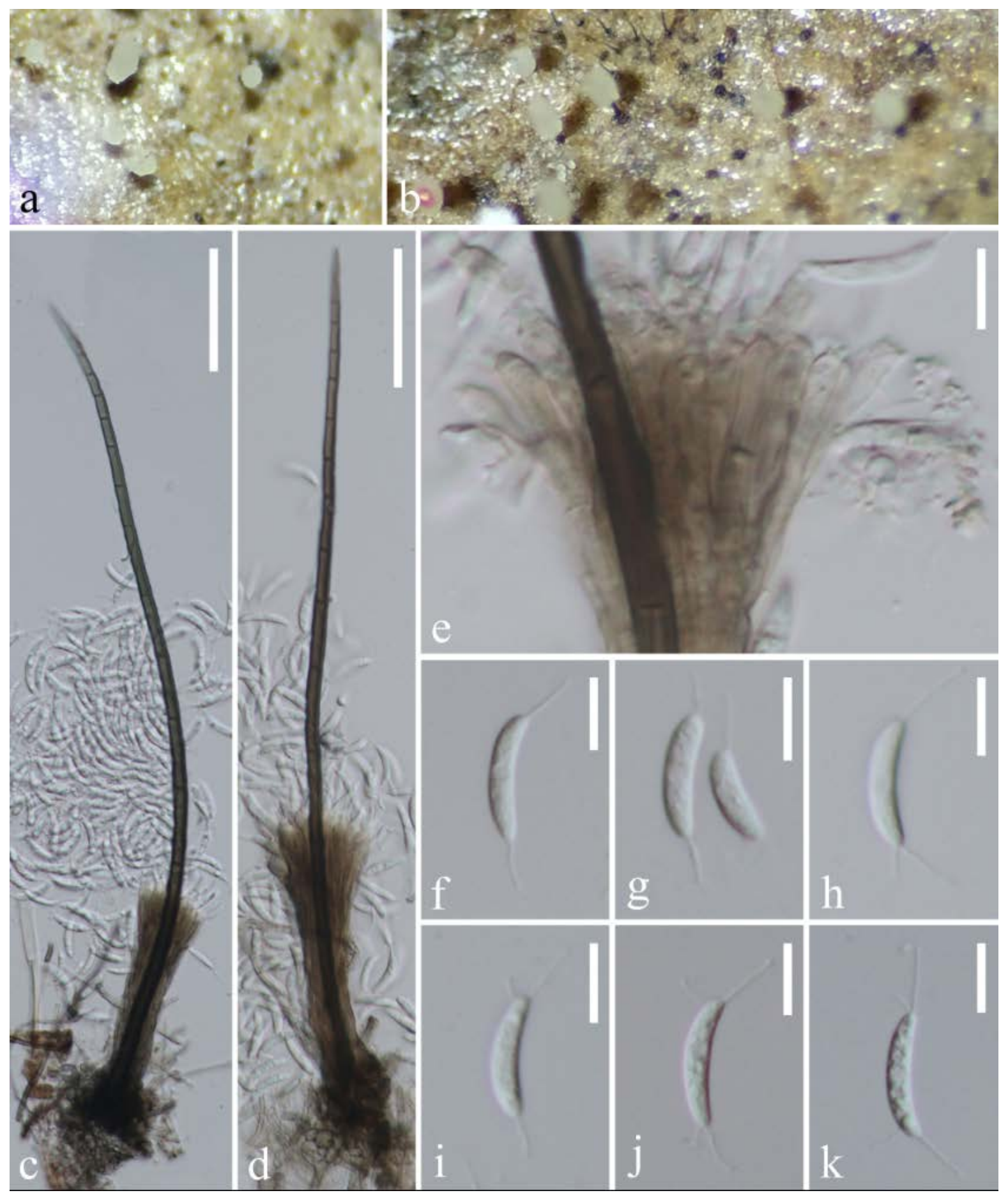

Figure 10 - Menisporopsis dushanensis (MFLU 19-0213, holotype) a, b Conidiophores on the host surface. c, d Conidiophores, setae, conidiogenous cells and conidia. e Conidiogenous cells. $\mathrm{f}-\mathrm{k}$ Conidia. Scale bars: $\mathrm{c}, \mathrm{d}=50 \mu \mathrm{m}, \mathrm{e}-\mathrm{k}=10 \mu \mathrm{m}$.

Saprobic on decaying plant material. Asexual morph: Colonies on plant substrate effuse, scattered, white to pale brown. Mycelium partly immersed, composed of brown hyphae. Conidiophores mononematous, macronematous, erect, straight or flexuous, unbranched, septate, smooth, dark brown at the base becoming light brown towards the apex, cylindrical, 155-405 $\mu \mathrm{m}$ long, 5.6-10.6 $\mu \mathrm{m}$ wide. Conidiogenous cells polyblastic, integrated, terminal, sympodial, pale brown, cylindrical. Conidia aggregated in slimy mass at apex of the conidiophores, acrogenous, smooth, hyaline, 1-3-septate, with guttules, cylindrical, oblong, sometimes slightly curved, with a short and hyaline appendage at each end, 15.8-20.9 $\times 6-8.3 \mu \mathrm{m}(\mathrm{av} .=19.2 \times 7.2 \mu \mathrm{m}, \mathrm{n}=30)$. Sexual morph: Undetermined. 
Material examined - THAILAND, Nan Province, Pua District, Phu Kha, on decaying plant, 4 August 2017, Chuan-Gen Lin, NAN 1-31 (MFLU 19-0218, holotype), ex-type living culture MFLUCC 18-0153.

Notes - Multiguttulispora is similar to Anacacumisporium Y.R. Ma \& X.G. Zhang, which was introduced by Ma et al. (2016) and typified by A. appendiculatum Y.R. Ma \& X.G. Zhang, by having mononematous, macronematous, unbranched, septate, dark brown to light brown and cylindrical conidiophores, integrated, terminal and cylindrical conidiogenous cells that produce septate conidia with an appendage at the tip and base and conidia aggregated in slimy masses at the conidiogenous loci. However, Multiguttulispora differs from Anacacumisporium by having sympodial conidiophores and hyaline and guttulate conidia, whereas the conidiophores are determinate and conidia are brown to dark brown or bicolorous in Anacacumisporium. Multiguttulispora is also similar to Cacumisporium Preuss by having cylindrical conidia with guttules, but Multiguttulispora has conidia with an appendage at both ends. A key to Anacacumisporium and similar genera was provided by Ma et al. (2016), which indicates that our fungus appears to be distinct from presently known genera. Phylogenetically, Multiguttulispora formed a separate clade within the family Chaetosphaeriaceae on the tree generated based on the combined LSU and ITS sequence data (Fig. 1). Therefore, we introduce a new genus, Multiguttulispora, to accommodate this distinct fungus $M$. sympodialis.

Tainosphaeria aseptata C.G. Lin \& J.K. Liu, sp. nov.

Fig. 12

Index Fungorum number: IF556713; Facesoffungi number: FoF06294

Etymology - Referring to the aseptate conidia.

Holotype - MFLU 19-0208

Saprobic on decaying wood. Asexual morph: Colonies on plant substrate effuse, aggregate, brown, with a hyaline, glistening conidial mass, hairy, short. Conidiophores mononematous, macronematous, erect, straight or flexuous, unbranched, septate, smooth, dark brown at the base, paler towards the apex, often with percurrent proliferations, cylindrical, 65-135 $\mu \mathrm{m}$ long, 3.5-5.5 $\mu \mathrm{m}$ wide at the base. Conidiogenous cells monophialidic, integrated, terminal, with conspicuous collarettes, pale brown to subhyaline, cylindrical, 28-49 $\mu \mathrm{m}$ long, 3.3-4.8 $\mu \mathrm{m}$ wide, narrowing to 1.2-2.8 $\mu \mathrm{m}$ just below the collarette. Conidia aggregated in slimy mass at the apex of the conidiophore, acrogenous, smooth, hyaline, aseptate, straight to curved, long fusiform, 15.7-23.3 $\times$ 3.8-4.5 $\mu \mathrm{m}($ av. $=20.6 \times 4.1 \mu \mathrm{m}, \mathrm{n}=20)$. Sexual morph: Undetermined.

Material examined - CHINA, Guizhou Province, Qiannan Buyi Miao Autonomous Prefecture, Dushan County, Guizhou Zilinshan National Forest Park (Shengou District), unnamed road, on decaying wood, 6 July 2018, Chuan-Gen Lin, DS 1-31 (MFLU 19-0208, holotype; HKAS 105130, isotype), ex-type living culture GZCC 18-0044.

Notes - Tainosphaeria aseptata is similar to Dictyochaeta heteroderae (Morgan-Jones) Carris \& Glawe and D. lunulospora (Hewings \& J.L. Crane) Hol.-Jech. by having mononematous conidiophores without fertile lateral branches, lacking setae, and aseptate, curved conidia without setulae. However, the conidia of D. heteroderae $(9-13 \times 3-4 \mu \mathrm{m})$ and $D$. lunulospora $(8.8-12.0 \times$ $0.8-1.0 \mu \mathrm{m})$ are smaller than those of T. aseptata $(15.7-23.3 \times 3.8-4.5 \mu \mathrm{m})$ and they are also guttulate. The phylogenetic results (Fig. 1) showed that our isolate clustered together with species of Tainosphaeria and Anacacumisporium. Tainosphaeria aseptata (GZCC 18-0044) formed a separate clade and sister to Anacacumisporium. However, this clade is not stable. Anacacumisporium is characterized by pigmented, transversely septate, appendaged conidia and conidiophores that are brown, macronematous, mononematous and that bear one (or more) integrated phialides at the tip (Ma et al. 2016). Our fresh specimen does not fit with the generic concept of Anacacumisporium. In addition, Tainosphaeria aseptata is distinguishable from all known asexual morph Tainosphaeria species. Therefore, $T$. aseptata is introduced as a new species based on both morphology and phylogeny. 


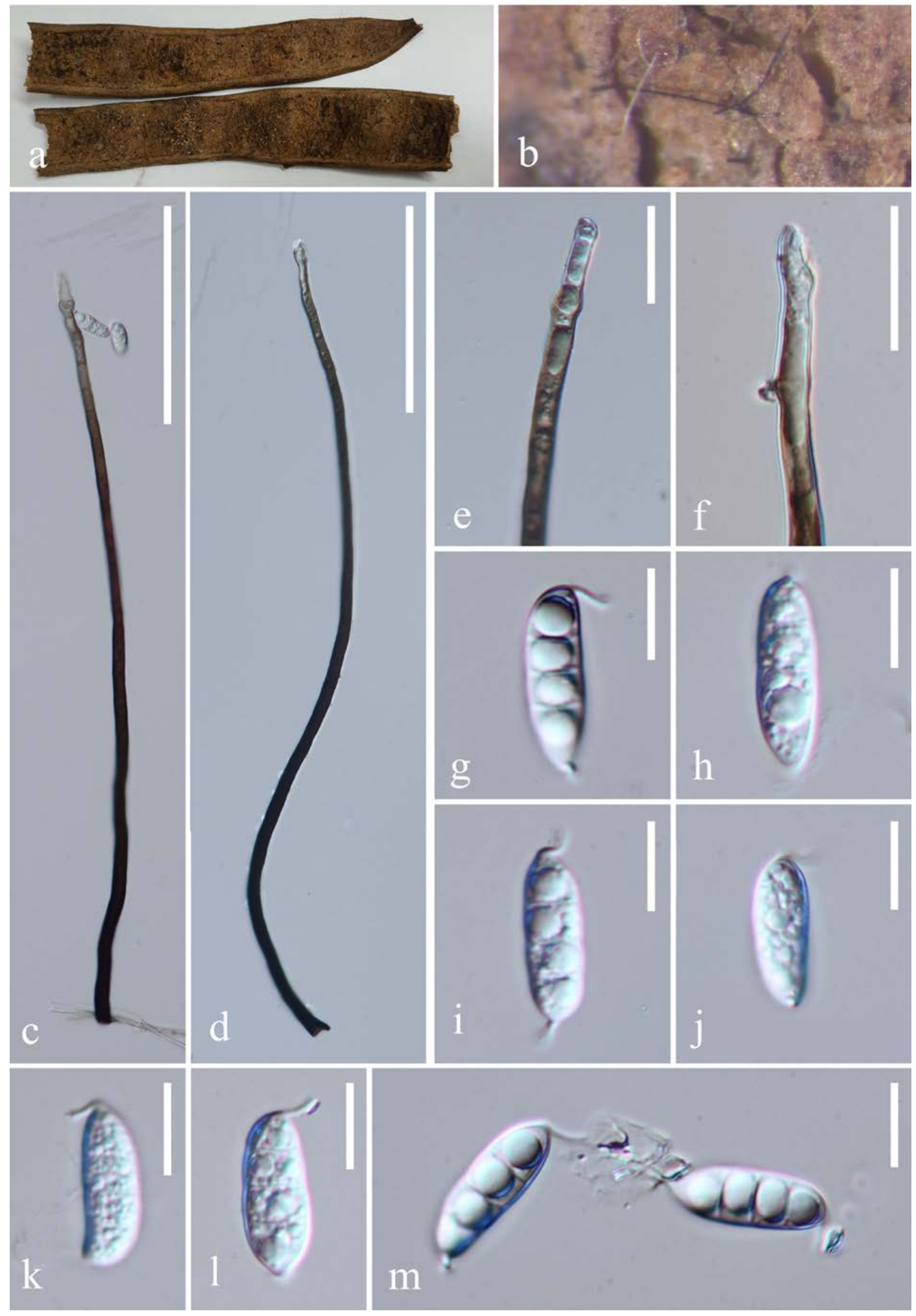

Figure 11 - Multiguttulispora sympodialis (MFLU 19-0218, holotype) a Host material. b Conidiophores on the host surface. c, d Conidiophores and conidiogenous cells. e, f Conidiogenous cells. g-m Conidia. Scale bars: $c, d=100 \mu \mathrm{m}, \mathrm{e}, \mathrm{f}=20 \mu \mathrm{m}, \mathrm{g}-\mathrm{m}=10 \mu \mathrm{m}$. 


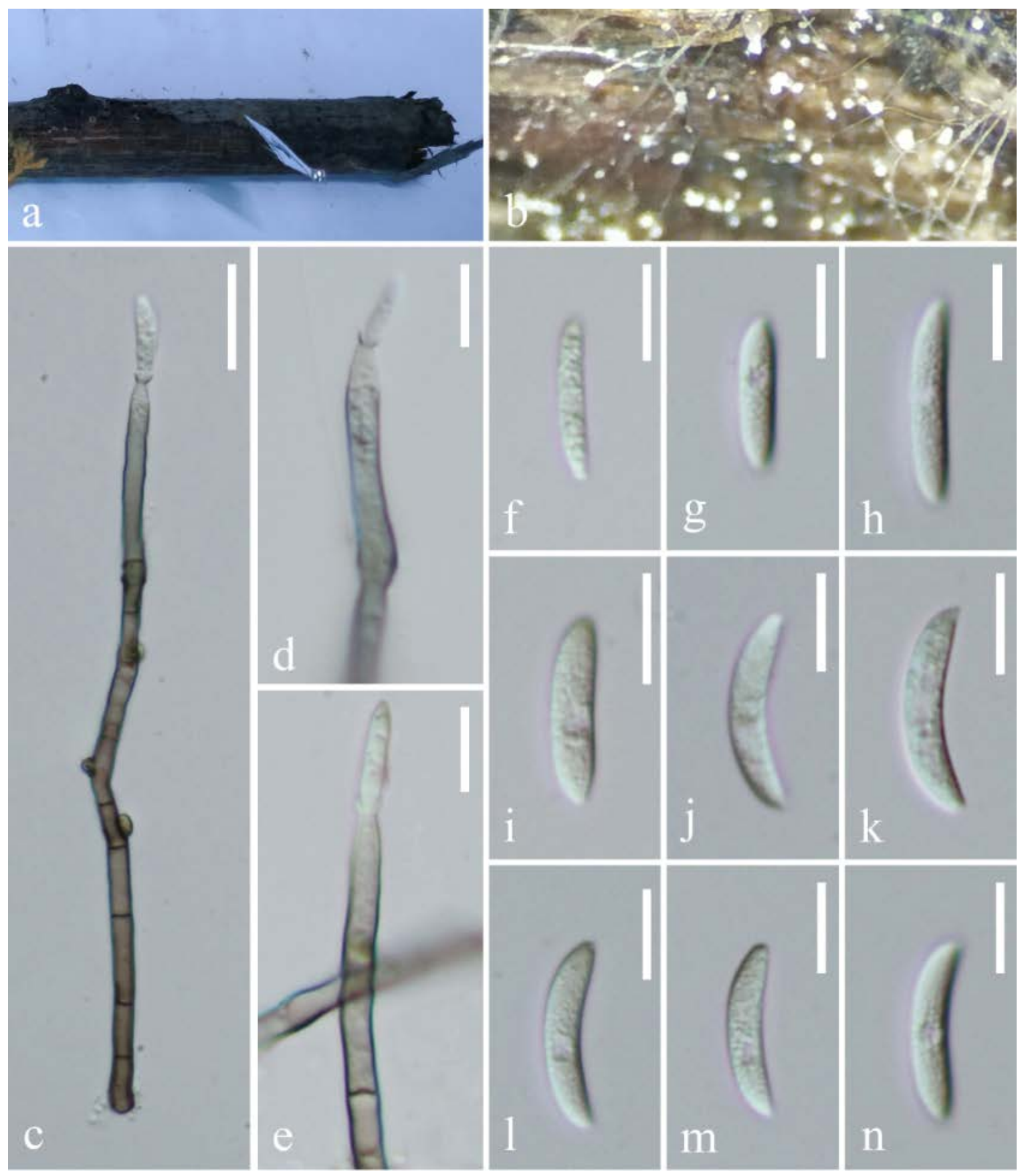

Figure 12 - Tainosphaeria aseptata (MFLU 19-0208, holotype) a Host material. b Conidiophores on the host surface. c Conidiophore and conidiogenous cell with conidium. d, e Conidiogenous cells. $\mathrm{f}-\mathrm{n}$ Conidia. Scale bars: $\mathrm{c}=20 \mu \mathrm{m}, \mathrm{d}-\mathrm{n}=10 \mu \mathrm{m}$.

Tainosphaeria monophialidica C.G. Lin \& J.K. Liu, sp. nov.

Fig. 13

Index Fungorum number: IF556714; Facesoffungi number: FoF06295

Etymology - Referring to the monophialidic conidiogenous cells.

Holotype - MFLU 19-0217

Saprobic on decaying wood. Asexual morph: Colonies on plant substrate effuse, aggregated, brown, as a hyaline, glistening conidial mass, hairy, short. Conidiophores mononematous, macronematous, erect, straight or slightly flexuous, unbranched, septate, smooth, pale brown at the base becoming subhyaline towards the apex, often with percurrent proliferations, sometimes reduced to conidiogenous cells, cylindrical, 33-120 $\mu \mathrm{m}$ long, 2.7-4.8 $\mu \mathrm{m}$ wide. Conidiogenous cells monophialidic, integrated, terminal, percurrent, with conspicuous collarettes, subhyaline, cylindrical, 20-46 $\mu \mathrm{m}$ long, 2.7-4.3 $\mu \mathrm{m}$ wide, narrowing to $1-2 \mu \mathrm{m}$ just below the collarette. 
Conidia aggregated in slimy mass at the apex of the conidiophores, acrogenous, smooth, hyaline, aseptate, straight to slightly curved, ellipsoidal, long fusiform, $14.0-19 \times 2.3-3.5 \mu \mathrm{m}$ (av. $=16.6 \times$ $3.0 \mu \mathrm{m}, \mathrm{n}=40$ ), with $7-10 \mu \mathrm{m}$ long hair-like appendage at both ends. Sexual morph: Undetermined.
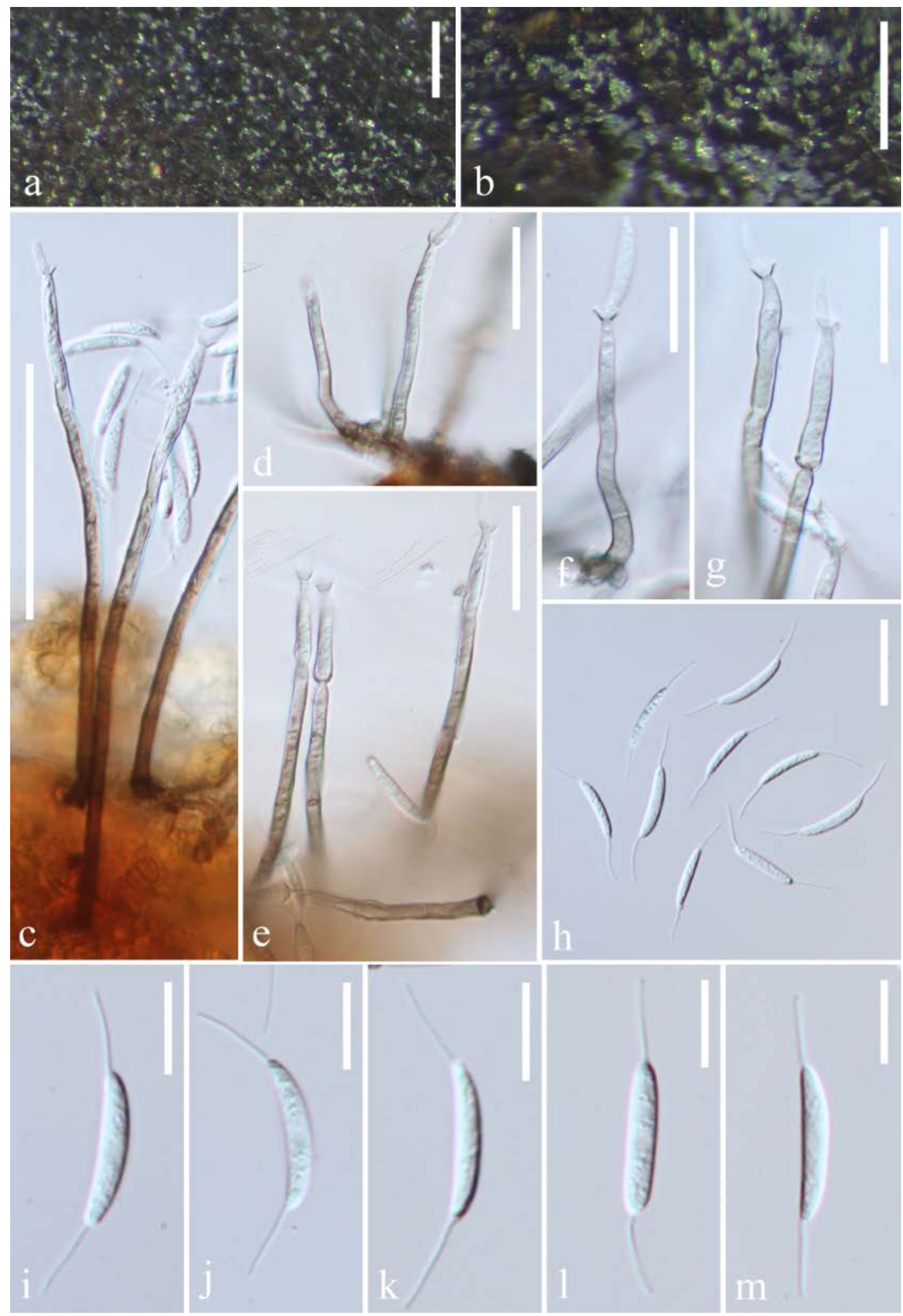

Figure 13 - Tainosphaeria monophialidica (MFLU 19-0217, holotype) a, b Conidiophores on the host surface. c-e Conidiophores and conidiogenous cells. $\mathrm{f}-\mathrm{g}$ Conidiogenous cells. $\mathrm{h}-\mathrm{m}$ Conidia. Scale bars: $\mathrm{a}, \mathrm{b}=200 \mu \mathrm{m}, \mathrm{c}=10 \mu \mathrm{m}, \mathrm{d}-\mathrm{h}=20 \mu \mathrm{m}, \mathrm{i}-\mathrm{m}=10 \mu \mathrm{m}$. 
Material examined - THAILAND, Nan, Pua District, Phu Kha, on decaying wood, 4 August 2017, Chuan-Gen Lin, NAN 1-3 (MFLU 19-0217, holotype), ex-type living culture MFLUCC 180146.

Notes - Tainosphaeria monophialidica is similar to Dictyochaeta tumidospora Kuthub. \& Nawawi by lacking setae, having mononematous conidiophores, well-developed and funnel-shaped collarettes, and aseptate, ellipsoidal, not typically curved conidia with long setulae at both ends. However, T. monophialidica has smaller conidia and shorter conidiophores than D. tumidiospora, and its conidiogenous cells are monophialidic rather than polyphialidic as in D. tumidiospora. The phylogenetic results (Fig. 1) showed that our isolate clustered together with other Tainosphaeria species but can be recognized as a distinct lineage. Tainosphaeria monophialidica is sister to $T$. jonesii (GZCC 16-0053 and GZCC 16-0065), but differs from T. jonesii by its conidiophores sometimes reduced to conidiogenous cells. In addition, the conidia are guttulate in T. jonesii. Based on the morphology and phylogeny, T. monophialidica is introduced as a new species.

\section{Discussion}

Taxa in the Chaetosphaeriaceae family are common on wood in terrestrial habitats and some are fungicolous while others are frequently recorded on submerged wood in freshwater (Goh \& Hyde 1996, Hyde \& Goh 1998, Ho et al. 2002, Maharachchikumbura et al. 2016, Sun et al. 2019). Réblová et al. (1999) accepted seven sexual genera and 13 asexual genera. Molecular data has allowed a better taxonomic understanding of the asexual morph genera (Shenoy et al. 2007) and various genera have been placed in this family (Table 2). In the most recent treatment, 38 genera were accepted (Wijayawardene et al. 2017, 2018). However, this family needs a detailed study of both asexual and sexual morphs of the various genera and a comparison with similar genera in other families. Several new Chaetosphaeriaceae species have been introduced in recent studies and updated phylogenetic trees have been provided (e.g. Li et al. 2016, Liu et al. 2016, Tibpromma et al. 2018, Yang et al. 2018, Hyde et al. 2019). Here we discuss some of the genera assigned to the Chaetosphaeriaceae.

Chaetosphaeria was introduced by Tulasne \& Tulasne (1863) for C. innumera Berk. \& Broome ex Tul. \& C. Tul. The asexual morphs of this genus are diverse. Seifert et al. (2011) listed 19 genera that are linked to Chaetosphaeria, viz., Cacumisporium, Catenularia Grove, Chalara (Corda) Rabenh., Chloridium, Codinaea Maire, Cryptophiale, Craspedodidymum Hol.-Jech., Custingophora Stolk, Hennebert \& Klopotek, Dictyochaeta, Exserohilum K.J. Leonard \& Suggs, Fusichalara S. Hughes \& Nag Raj, Gonytrichum Nees \& T. Nees, Kylindria DiCosmo, S.M. Berch \& W.B. Kendr., Menispora Pers., Menisporopsis S. Hughes, Phaeostalagmus W. Gams, Phialophora Medlar, Sporoschisma Berk. \& Broome and Zanclospora S. Hughes \& W.B. Kendr. In this study, the phylogenetic results (Fig. 1) also confirmed some of these connections, e.g. Chloridium, Codinaea, Dictyochaeta, Exserohilum, Gonytrichum and Menispora.

The genera Codinaea, Codinaeopsis, Dictyochaeta and Dictyochaetopsis are problematic. A brief historical review of these four genera was provided by Li et al. (2012) and Réblová (2000). In this study, we treat Codinaea, Dictyochaeta and Dictyochaetopsis as distinct genera and accept the treatment of Whitton et al. (2000) to synonymize Codinaeopsis under Dictyochaetopsis to prevent ongoing confusion until a monographic study on these genera is performed in the future.

This study involves a phylogenetic analysis based on combined SSU, ITS and LSU sequence data to assess taxonomy of Kionochaeta (Fig. 2). Phylogenys showed that Kionochaeta is polyphyletic and species are phylogenetically located in two groups (Kionochaeta 1 and Kionochaeta 2) (Fig. 2). A synopsis of Kionochaeta species is provided here (Table 3).

The status of Menisporopsis within Chaetosphaeriaceae was confirmed by Liu et al. (2016). Tibpromma et al. (2018) introduced the new species $M$. pandanicola and we introduce two new species. Twelve species are accepted within Menisporopsis. A synopsis of Menisporopsis species is provided here (Table 4).

Ellisembia Subram. was synonymized under Sporidesmium Link by Su et al. (2016). The main difference between these two genera is that the conidia of Ellisembia are distoseptate, while 
those of Sporidesmium are euseptate. However, Su et al. (2016) suggested that this difference was not significant. We included E. brachypus (HKUCC 10555) in our phylogenetic analysis, and it clustered within Chaetosphaeriaceae (Fig. 1). Hyde et al. (2019) introduced a new species Ellisembia aurea Réblová \& J. Fourn. and placed it within Chaetosphaeriaceae. Luo et al. (pers. comm.) suggested to use Sporidesmium brachypus rather than Ellisembia brachypus. Shenoy et al. (2006) confirmed that Sporidesmium is polyphyletic based on combined LSU and RPB2 sequence data.

The placements of some genera within Chaetosphaeriaceae are discussed here and some of them are re-assigned. Samuels et al. (1997) introduced the genus Ascocodinaea with a Codinaea asexual morph and placed this genus within family Lasiosphaeriaceae. Réblová et al. (1999) placed Ascocodinaea within Chaetosphaeriaceae based on the asci, ascospores and perithecial morphology and the Dictyochaeta asexual morph. However, this treatment has not been universally accepted (Huhndorf et al. 2004, Lumbsch \& Huhndorf 2007b, 2010, Wijayawardene et al. 2012, 2017, 2018, Maharachchikumbura et al. 2015, 2016). In our phylogenetic analysis (Fig. 1), the strain (GJS 95184) of A. stereicola clustered outside of the Chaetosphaeriaceae (data not shown), and we exclude Ascocodinaea from the family.

Bahusutrabeeja was introduced by Subramanian \& Bhat (1977), and typified by B. dwaya Subram. \& Bhat, but its taxonomic placement was uncertain. Shenoy et al. (2010) placed it within family Botryosphaeriaceae (Botryosphaeriales, Dothideomycetes) based on phylogenetic analysis of LSU sequence data of a fresh specimen of B. dwaya from India. This treatment was accepted by many authors (Hyde et al. 2011, Seifert et al. 2011, Wijayawardene et al. 2012, Slippers et al. 2017, Burgess et al. 2019). However, in the present study, LSU and ITS sequence data analysis of $B$. dwaya ex-type strain (CBS 261.77) shows that it is phylogenetically related to Dictyochaeta spp. in Chaetosphaeriaceae (Fig. 1). Bahusutrabeeja is similar to some genera (Codinaea, Dictyochaeta, Dinemasporium, Menispora, Thozetella) in Chaetosphaeriaceae by having phialidic conidiogenous cells and aseptate conidia with slender appendages (Subramanian \& Bhat 1977, Réblová et al. 1999, Seifert et al. 2011, Li et al. 2014, Gao et al. 2015). We suggest to assign the genus Bahusutrabeeja to the family Chaetosphaeriaceae based on morphology and phylogenetic analysis.

Calvolachnella Marinc., T.A. Duong \& M.J. Wingf. was introduced by Hernández-Restrepo et al. (2016) to accommodate C. guaviyunis (Marinc., T.A. Duong, M.J. Wingf. \& C.A. Perez) Marinc., T.A. Duong, M.J. Wingf., which was previously known as Pseudolachnella guaviyunis. This genus is characterized by having acervular conidiomata, absence of setae in conidiomata, pale brown, septate and branched conidiophores, phialidic conidiogenous cells that produce fusiform, hyaline to pale brown conidia (Hernández-Restrepo et al. 2016). Hernández-Restrepo et al. (2016) placed this genus in Chaetosphaeriales genera incertae sedis. Our phylogenetic results show that the ex-type strain of $C$. guaviyunis forms a separate clade and sister to Dictyochaeta group within the Chaetosphaeriaceae (Fig. 1). Therefore, we assign Calvolachnella within the family Chaetosphaeriaceae.

The genus Conicomyces was established by Sinclair et al. (1983) to accommodate a distinctive synnematous hyphomycete. Hashimoto et al. (2015) suggested to place this genus within Chaetosphaeriaceae based on its morphological similarity with Pseudolachnea and its relatives. The first phylogenetic analysis of the genus Conicomyces was performed by Liu et al. (2015) and confirmed its placement within Chaetosphaeriaceae.

The genus Exserticlava was established by Hughes (1978) and has a Chaetosphaeria sexual morph (Matsushima 1985, Réblová \& Seifert 2003, Fernández \& Huhndorf 2005, Seifert et al. 2011). Unpublished sequence data of E. vasiformis (Matsush.) S. Hughes (TAMA 450) was included in our analysis, and it showed close phylogenetic relationship with Chaetosphaeria capitata Sivan. \& H.S. Chang (SMH 3239) with high support value (99\% MLBS /0.95 PP) (Fig. 1). Exserticlava vasiformis was found on the holotype specimen of C. capitata but without a common mycelial link between them (Sivanesan \& Chang 1995). Fernández \& Huhndorf (2005) confirmed the sexual-asexual connection between C. capitata and E. vasiformis by culturing ascospores in vitro We confirm this connection by phylogenetic analysis (Fig. 1). 
Table 2 Genera accepted within family Chaetosphaeriaceae by various authors.

\begin{tabular}{|c|c|c|c|c|c|}
\hline Réblová et al. (1999)a & Lumbsch \& Huhndorf (2010)a & Seifert et al. $(2011)^{b}$ & Maharachchikumbura et al. (2015) & Maharachchikumbura et al. (2016) & Wijayawardene et al. (2018) \\
\hline Ascocodinaea & Ascochalara & Cacumisporium & Ascochalara & Ascochalara & Adautomilanezia \\
\hline Chaetosphaeria & Australiasca & Catenularia & Brunneodinemasporium & Brunneodinemasporium & Anacacumisporium \\
\hline Melanochaeta & Chaetosphaeria & Chloridium & Catenularia & Catenularia & Ascochalara \\
\hline Melanopsammella & =Paragaeumannomyces & Codinaea & Chaetosphaeria & Chaetosphaeria & Brunneodinemasporium \\
\hline Porosphaerella & Lecythothecium & Craspedodidymum & Chloridium & Chloridium & Catenularia \\
\hline Porosphaerellopsis & Melanochaet & Cryptophiale & Codinaea & Codinaea & Chaetosphaeria \\
\hline \multirow[t]{33}{*}{ Striatosphaeria } & Melanopsammella & Custingophora & Codinaeopsis & Codinaeopsis & Chloridium \\
\hline & Porosphaerella & Dictyochaeta & Craspedodidymum & Craspedodidymum & Codinaea \\
\hline & Striatosphaeria & Exserticlava & Cryptophiale & Cryptophiale & Conicomyces \\
\hline & Tainosphaeri & Fusichalara & Dendrophoma & Dendrophoma & Craspedodidymum \\
\hline & Zignoëlla & Gonytrichum & Dictyochaeta & Dictyochaeta & Cryptophiale \\
\hline & & Hyphopolynema & Dictyochaetopsis & Dictyochaetopsis & Dendrophoma \\
\hline & & Janetia & Dinemasporium & Dinemasporium & Dictyochaeta \\
\hline & & Kylindria & Exserticlava & Exserticlava & Dinemasporium \\
\hline & & Menispora & Gonytrichum & Gonytrichum & Eucalyptostroma \\
\hline & & Menisporopsis & Hemicorynespora & Hemicorynespora & Exserticlava \\
\hline & & Paliphora & Infundibulomyces & Infundibulomyces & Gonytrichum \\
\hline & & Phaeostalagmus & Kionochaeta & Kionochaeta & Hemicorynespora \\
\hline & & Pleurothecium & Lecythothecium & Lecythothecium & Infundibulomyces \\
\hline & & Rattania & Melanopsammella & Melanopsammella & Kionochaeta \\
\hline & & Sporoschisma & Menispora & Menispora & Lecythothecium \\
\hline & & Sporoschismopsis & Miyoshiella & Miyoshiella & Melanopsammella \\
\hline & & Thozetella & Phaeostalagmus & Neopseudolachnella & Menispora \\
\hline & & Zanclospora & Phialogeniculata & Phaeostalagmus & Menisporopsis \\
\hline & & & Pseudobotrytis & Phialogeniculata & Miyoshiella \\
\hline & & & Pseudolachnea & Pseudobotrytis & Neopseudolachnella \\
\hline & & & Pyrigemmula & Pseudodinemasporium & Pseudobotrytis \\
\hline & & & Rattania & Pseudolachnea & Pseudodinemasporium \\
\hline & & & Sporoschisma & Pyrigemmula & Pseudolachnea \\
\hline & & & $=$ Melanochaeta & Rattania & Pseudolachnella \\
\hline & & & Striatosphaeria & Sporoschisma & Pyrigemmula \\
\hline & & & Tainosphaeria & $=$ Melanochaeta & Rattania \\
\hline & & & Thozetella & Striatosphaeria & Sporoschisma \\
\hline & & & Umbrinosphaeria & Tainosphaeria & $=$ Melanochaeta \\
\hline & & & Zanclospora & Thozetella & Striatosphaeria \\
\hline & & & Zignoëlla & Umbrinosphaeria & Tainosphaeria \\
\hline & & & & Zanclospora & Thozetella \\
\hline & & & & Zignoëlla & Umbrinosphaeria \\
\hline & & & & & Zanclospora \\
\hline
\end{tabular}

${ }^{\mathrm{a}}$ Only included the sexual genera, ${ }^{\mathrm{b}}$ Only included the hyphomycetous genera. 
Table 3 Synopsis of Kionochaeta species.

\begin{tabular}{|c|c|c|c|c|}
\hline & Conidiophores & Fertile branches & Conidiogenous cells & Conidia \\
\hline K. aristata & Simple, subulate, smooth, $240-320 \mu \mathrm{m}$ high & $\begin{array}{l}\text { Borne sub-medianly on } \\
\text { the conidiophore }\end{array}$ & $\begin{array}{l}\text { Lageniform, with a } \\
\text { conspicuous, cup-shaped } \\
\text { collarette at the apex. }\end{array}$ & $\begin{array}{l}\text { Cylindrical to broadly fusiform, slightly } \\
\text { curved, with a setula } 6.0-8.5 \mu \mathrm{m} \text { long at each } \\
\text { end, hyaline, smooth, non-septate, } 2-4 \\
\text { guttulate, } 10-12(-14) \mu \mathrm{m} \text { long, } 1.5-2.0 \mu \mathrm{m} \\
\text { wide. }\end{array}$ \\
\hline K. australiensis & $\begin{array}{l}\text { Setiform, smooth, up to 9-septate, subulate, } 200-300 \mu \mathrm{m} \text { long, } \\
\text { unbranched or occasionally with a single lateral setiform branch } \\
\text { arising from the middle of the central element; apex of central } \\
\text { setiform element and lateral setiform branches fertile; }\end{array}$ & $\begin{array}{l}\text { Median to submedian on } \\
\text { the conidiophore }\end{array}$ & $\begin{array}{l}\text { Ampulliform or } \\
\text { lageniform, subhyaline to } \\
\text { hyaline, monoblastic }\end{array}$ & $\begin{array}{l}\text { Ellipsoidal to fusiform, hyaline, smooth, } 4-5 \\
\times 1-1.5 \mu \mathrm{m}\end{array}$ \\
\hline K. castaneae & $\begin{array}{l}\text { Setiform, dark brown, smooth, sometimes branched above the } \\
\text { fertile region, sometimes with secondly fertile region at the apex } \\
\text { of the main axis, } 265-320 \mu \mathrm{m} \text { long, } 7-9.5 \mu \mathrm{m} \text { wide at the base; } \\
\text { when branched, terminal branches dark brown, } 45-105 \mu \mathrm{m} \text { long, } \\
2.5-5.5 \mu \mathrm{m} \text { wide at the base, acutely pointed above }\end{array}$ & $\begin{array}{l}\text { Middle part of the } \\
\text { conidiophores, rarely } \\
\text { with the secondarily } \\
\text { fertile region at the apex } \\
\text { of the main axis }\end{array}$ & $\begin{array}{l}\text { Narrowly ellipsoid, pale } \\
\text { brown }\end{array}$ & $\begin{array}{l}\text { Hyaline, smooth, lunate, cylindrical, aseptate, } \\
5.1-6.5 \times 1.2-1.8 \mu \mathrm{m}\end{array}$ \\
\hline K. filamentosa & $\begin{array}{l}\text { Unbranched, smooth, multiseptate, cylindrical, 800-1200 } \mu \mathrm{m} \\
\text { long }\end{array}$ & Terminal & $\begin{array}{l}\text { Monoblastic, ampulliform } \\
\text { or lageniform, hyaline to } \\
\text { pale brown }\end{array}$ & $\begin{array}{l}\text { Hyaline, narrowly ellipsoidal to fusiform, } 0 \text { - } \\
\text { or } 1 \text {-septate, smooth, } 10-16 \times 1.5-3 \mu \mathrm{m} \text { (av. } \\
=14 \times 2 \mu \mathrm{m}, \mathrm{n}=25)\end{array}$ \\
\hline K. ivoriensis & $\begin{array}{l}\text { Setiform, subulate, smooth, } 70-150 \mu \mathrm{m} \text { high, } 4.5-5.5 \mu \mathrm{m} \text { wide, } \\
12.5-14.0 \mu \mathrm{m} \text { wide at the base, up to } 11 \text {-septate, unbranched }\end{array}$ & $\begin{array}{l}\text { Terminal and/or situated } \\
\text { just below the middle of } \\
\text { the main axis }\end{array}$ & $\begin{array}{l}\text { Ampulliform to short } \\
\text { lageniform, with an } \\
\text { indistinct collarette. }\end{array}$ & Aseptate, cylindrical, 3.4-5.0 × 1.5-2.5 $\mu \mathrm{m}$ \\
\hline K. keniensis & $\begin{array}{l}\text { Setiform, subulate, smooth, } 200-260 \mu \mathrm{m} \text { high, up to } 8 \text {-septate, } \\
\text { branched }\end{array}$ & $\begin{array}{l}\text { Just below the middle of } \\
\text { the conidiophores }\end{array}$ & $\begin{array}{l}\text { Cylindrical, with an } \\
\text { indistinct collarette }\end{array}$ & $\begin{array}{l}\text { Aseptate, setulate, cylindrical to broadly } \\
\text { fusiform, slightly curved, (11-)12-16 } \mu \mathrm{m} \\
\text { long (excluding the setulae), 1.5-2.5 } \mu \mathrm{m} \text { wide }\end{array}$ \\
\hline K. malaysiana & Simple, subulate, smooth, 3-4-septate, 200-250 $\mu \mathrm{m}$ high & $\begin{array}{l}\text { Borne sub-medianly on } \\
\text { the conidiophore at two } \\
\text { loci }\end{array}$ & $\begin{array}{l}\text { Obpyriform, with an } \\
\text { indistinct collarette at the } \\
\text { apex }\end{array}$ & $\begin{array}{l}\text { Narrowly clavate to fusiform, slightly curved, } \\
\text { hyaline, smooth, non-septate, (11-)14-16(- } \\
\text { 17) } \mu \mathrm{m} \text { long, } 1.0(-1.5) \mu \mathrm{m} \text { wide }\end{array}$ \\
\hline K. microspora & $\begin{array}{l}\text { Setiform, smooth, unbranched, septate, dark brown at the base, } \\
\text { paler towards the apex, cylindrical, swollen at the base, } 40-305 \\
\mu \mathrm{m} \text { long, } 3.3-10.5 \mu \mathrm{m} \text { wide at the base }\end{array}$ & $\begin{array}{l}\text { At the apex of the } \\
\text { conidiophores, rarely at } \\
\text { the middle of the } \\
\text { conidiophores with a } \\
\text { secondary fertile region } \\
\text { at the conidiophore apex }\end{array}$ & $\begin{array}{l}\text { Narrowly ellipsoid, } \\
\text { ampulliform, subhyaline to } \\
\text { pale brown }\end{array}$ & $\begin{array}{l}\text { Hyaline, smooth, lunate, cylindrical or } \\
\text { clavate, aseptate, } 4.2-6.4 \times 1.5-2.3 \mu \mathrm{m}\end{array}$ \\
\hline
\end{tabular}


Table 3 Continued.

\begin{tabular}{|c|c|c|c|c|}
\hline & Conidiophores & Fertile branches & Conidiogenous cells & Conidia \\
\hline K. nanophora & $\begin{array}{l}\text { Setiform element up to } 10 \text {-septate, subulate, up to } 320 \mu \mathrm{m} \text { tall, } \\
\text { occasionally fertile at the apex; conidiophores smooth, up to } 2-4 \\
\text { septate, up to } 160 \mu \mathrm{m} \text { tall }\end{array}$ & $\begin{array}{l}\text { At the apex of the } \\
\text { shorter conidiophore }\end{array}$ & $\begin{array}{l}\text { Ampulliform to lageniform } \\
\text { with an indistinct collarette } \\
\text { at the apex. }\end{array}$ & $\begin{array}{l}\text { Fusiform, hyaline, smooth, non-septate, } 5-7 \times \\
0.8-1.2 \mu \mathrm{m}\end{array}$ \\
\hline K. pini & Simple, verrucose, up to 10 -septate, subulate, $120-180 \mu \mathrm{m}$ tall & $\begin{array}{l}\text { Situated in middle of } \\
\text { conidiophore }\end{array}$ & $\begin{array}{l}\text { Ampulliform to lageniform } \\
\text { or cylindrical; apical } \\
\text { collarettes inconspicuous. }\end{array}$ & $\begin{array}{l}\text { Fusiform to cylindrical, hyaline, smooth, } \\
\text { aseptate, } 10-12 \times 1.5-2 \mu \mathrm{m} \text {. }\end{array}$ \\
\hline K. pleomorpha & $\begin{array}{l}\text { Simple, up to 7-septate, } 50-100 \mu \mathrm{m} \text { long, cylindrical to } \\
\text { subcylindrical }\end{array}$ & $\begin{array}{l}\text { At the apex of } \\
\text { conidiophores }\end{array}$ & $\begin{array}{l}\text { Lageniform or globose } \\
\text { with short narrow neck, } \\
\text { hyaline to subhyaline }\end{array}$ & $\begin{array}{l}\text { Small, hyaline, bacillar, one-celled, smooth, } \\
\text { slimy, } 4-5 \times 1.5 \mu \mathrm{m}\end{array}$ \\
\hline K. pughii & $\begin{array}{l}\text { Smooth, up to } 16 \text {-septate, subulate, central element up to } 360 \mu \mathrm{m} \\
\text { high, branched; tips of central element and lateral branches } \\
\text { fertile }\end{array}$ & $\begin{array}{l}\text { Just below the middle of } \\
\text { conidiophores }\end{array}$ & $\begin{array}{l}\text { Ampulliform to } \\
\text { lageniform, with an } \\
\text { indistinct collarette at the } \\
\text { apex. }\end{array}$ & $\begin{array}{l}\text { Fusiform, hyaline, smooth, non-septate, 5.5- } \\
7.0 \times 1-1.2 \mu \mathrm{m}\end{array}$ \\
\hline K. ramifera & $\begin{array}{l}\text { Setiform, subulate, smooth or verruculose, up to } 300 \mu \mathrm{m} \text { high, up } \\
\text { to } 16 \text {-septate }\end{array}$ & $\begin{array}{l}\text { Just below the middle of } \\
\text { conidiophores }\end{array}$ & $\begin{array}{l}\text { Ampulliform to lageniform } \\
\text { or cylindrical, with an } \\
\text { indistinct collarette. }\end{array}$ & $\begin{array}{l}\text { Aseptate, narrowly clavate, slightly curved, } \\
(5-) 6-10(-12) \times 1.0-1.5(-2.0) \mu \mathrm{m}\end{array}$ \\
\hline K. spissa & Simple, subulate, smooth, $170-260 \mu \mathrm{m}$ high & $\begin{array}{l}\text { Borne sub-medianly on } \\
\text { the conidiophore at a } \\
\text { single locus }\end{array}$ & $\begin{array}{l}\text { Lageniform, with an } \\
\text { indistinct collarette at the } \\
\text { apex }\end{array}$ & $\begin{array}{l}\text { Narrowly clavate to fusiform, slightly curved, } \\
\text { hyaline, smooth, non-septate, } 4.5-6.5(-8.0) \times \\
1.0-1.5 \mu \mathrm{m}\end{array}$ \\
\hline K. virtuosa & $\begin{array}{l}\text { Setiform, subulate, smooth, 225-300 } \mu \mathrm{m} \text { high, up to } 17 \text {-septate, } \\
\text { unbranched }\end{array}$ & $\begin{array}{l}\text { Situated just below the } \\
\text { middle of the main axis }\end{array}$ & $\begin{array}{l}\text { Lageniform to cylindrical, } \\
\text { with a prominent collarette. }\end{array}$ & 1-septate, falcate, $20.5-34.5 \times 2.0-2.5 \mu \mathrm{m}$ \\
\hline
\end{tabular}

Table 4 Synopsis of Menisporopsis species.

\begin{tabular}{|c|c|c|c|c|}
\hline & Setae & Synnema width & Conidiophores & Conidia \\
\hline M. anisospora & $\begin{array}{l}200-425 \times 10-12 \\
\mu \mathrm{m}, 7-15 \text {-septate }\end{array}$ & $60-80 \mu \mathrm{m}$ & $\begin{array}{l}\text { Brown to dark brown, } 300-500 \times 2-3 \mu \mathrm{m} \text {, single } \\
\text { phialides } 2.5-4 \mu \mathrm{m} \text { wide }\end{array}$ & $\begin{array}{l}\text { Allantoid, fusiform, subsigmoid, sublunate, vermiform to irregular, } \\
\text { aseptate, hyaline, } 17-30 \times 2-6 \mu \mathrm{m}, 1 \text { apical setula, } 4-11 \mu \mathrm{m} ; 1 \text { basal } \\
\text { setula, 3-10 } \mu \mathrm{m} \text {; 1-2(3) lateral setula, } 7-10 \mu \mathrm{m}\end{array}$ \\
\hline M. breviseta & $\begin{array}{l}\text { 97-189 } \mu \mathrm{m} \text { long, } \\
2.7-5.4 \mu \mathrm{m} \text { thick }\end{array}$ & $6.5-12 \mu \mathrm{m}$ wide & Up to $182 \mu \mathrm{m}$ long & $\begin{array}{l}\text { Curved, cylindrical or fusiform, aseptate, hyaline, } 14.2-24.3 \times 2.4-3.6 \\
\mu \mathrm{m} \text {, with one or two setulae at each end, setulae } 4-9 \mu \mathrm{m} \text { long }\end{array}$ \\
\hline
\end{tabular}


Table 4 Continued.

\begin{tabular}{|c|c|c|c|c|}
\hline & Setae & Synnema width & Conidiophores & Conidia \\
\hline M. dushanensis & $207-455 \mu \mathrm{m}$ long & 8.5-22.5 $\mu \mathrm{m}$ wide & Up to $147 \mu \mathrm{m}$ long & $\begin{array}{l}\text { Curved, cylindrical or fusiform, aseptate, hyaline, } 14.0-20.6 \times 2.7-4.2 \\
\mu \mathrm{m} \text {; with one or two setulae at each end, setulae } 3-11 \mu \mathrm{m} \text { long }\end{array}$ \\
\hline M. kobensis & $\begin{array}{l}250-275 \mu \mathrm{m} \text { long, } \\
7.5-8.8 \mu \mathrm{m}, \text { septate }\end{array}$ & - & $\begin{array}{l}\text { Reduced to conidiogenous cells, } 75-90 \mu \mathrm{m} \text { long, } \\
\text { below } 2.5-3 \mu \mathrm{m} \text { wide, up slightly to } 3-3.5 \mu \mathrm{m} \\
\text { inflated, beneath the light brown, upper colorless, } \\
\text { single phialides }\end{array}$ & $\begin{array}{l}0(-1) \text {-septate, }(19-) 22.5-30(-32) \times 4.5 \mu \mathrm{m}, 1 \text { setula at each end, } 6-10 \mu \mathrm{m} \\
\text { long }\end{array}$ \\
\hline M. multisetulata & $\begin{array}{l}300-500 \times 6-10 \\
\mu \mathrm{m}, 10-15 \text {-septate }\end{array}$ & $22-40 \mu \mathrm{m}$ & $\begin{array}{l}\text { Brown, } 180-220 \times 1-2 \mu \mathrm{m} \text { wide, with } 3-4 \mu \mathrm{m} \\
\text { wide single phialides }\end{array}$ & $\begin{array}{l}\text { Allantoid, } 0 \text {-septate, } 12-18 \times 2.5-4 \mu \mathrm{m}, 2(3) \text { and } 3(4) \text { setulae on each } \\
\text { end, long one } 7-10 \mu \mathrm{m} \text {, short one } 2-4 \mu \mathrm{m}\end{array}$ \\
\hline $\begin{array}{l}\text { M. novae- } \\
\text { zelandiae }\end{array}$ & $\begin{array}{l}220-820 \times 7-10 \\
\mu \mathrm{m}, 17 \text {-septate }\end{array}$ & $14-21 \mu \mathrm{m}$ & Pale brown, single phialides $2.8-4.3 \mu \mathrm{m}$ wide & $\begin{array}{l}\text { Curved, } 1 \text {-septate, } 15-18 \times 2.4-3.1 \mu \mathrm{m}, 1 \text { setula at distal end and to one } \\
\text { side of the basal scar, } 4.3-5.7 \mu \mathrm{m} \text { long }\end{array}$ \\
\hline M. pandanicola* & $\begin{array}{l}344-375 \times 7-10.5 \\
\mu \mathrm{m}, \text { multi-septate }\end{array}$ & $14.5-23 \mu \mathrm{m}$ & Pale brown & $\begin{array}{l}\text { Lunate, conical at both ends, aseptate, guttulate, hyaline, } 17-22 \times 2-3 \mu \mathrm{m} \text {, } \\
(1-) 2 \text { setula at each end, } 4-12 \mu \mathrm{m} \text { long. }\end{array}$ \\
\hline M. pirozynskii & $\begin{array}{l}300-500 \times 5.5-6 \\
\mu \mathrm{m}, 10-15 \text {-septate }\end{array}$ & $15-27 \mu \mathrm{m}$ & $\begin{array}{l}\text { Brown, } 75-80 \times 1.5-2 \mu \mathrm{m} \text { wide, with } 2-2.5 \mu \mathrm{m} \\
\text { wide single phialides }\end{array}$ & $\begin{array}{l}\text { Allantoid, } 0 \text {-septate, } 16-20.5 \times 2-3.5 \mu \mathrm{m}, 2 \text { setulae at each end, terminal } \\
\text { one } 7-10 \mu \mathrm{m} \text {, subterminal one } 2.5-8 \mu \mathrm{m}\end{array}$ \\
\hline M. pleiosetosa & $\begin{array}{l}100-300 \times 2-4 \mu \mathrm{m} \\
10-15 \text {-septate }\end{array}$ & $30-40 \mu \mathrm{m}$ & $\begin{array}{l}\text { Brown, } 250 \times 3-4 \mu \mathrm{m} \text { wide, with } 1-2 \mu \mathrm{m} \text { wide } \\
\text { single phialide }\end{array}$ & $\begin{array}{l}\text { Ellipsoidal, } 0 \text {-septate, } 12-18 \times 4-5 \mu \mathrm{m}, 2-4 \text { setulae around the conidial } \\
\text { base and } 1 \text { at the apex, up to } 6 \mu \mathrm{m} \text { long }\end{array}$ \\
\hline M. profusa & $\begin{array}{l}150-250 \times 4.5-6 \\
\mu \mathrm{m}, 10-15 \text {-septate }\end{array}$ & $10 \mu \mathrm{m}$ & $\begin{array}{l}\text { Gold brown, polyphialidic, phialides } 3.5-5 \mu \mathrm{m} \\
\text { wide }\end{array}$ & Curved, 0 -septate, $12-15 \times 2-2.5 \mu \mathrm{m}, 1$ setula at each end, $4.5-6 \mu \mathrm{m}$ long \\
\hline M. theobromae & $\begin{array}{l}200-420 \times 5-7 \mu \mathrm{m} \\
21 \text {-septate }\end{array}$ & са. $10 \mu \mathrm{m}$ & Brown, monophialidic, phialides $2.5-3 \mu \mathrm{m}$ wide & 0 -septate, $14-18 \times 2-3 \mu \mathrm{m}, 1$ setula at each end, up to $10 \mu \mathrm{m}$ long \\
\hline M. trisetulosa & $\begin{array}{l}250-460 \times 5.5-7 \\
\mu \mathrm{m}, \text { about } 20- \\
\text { septate }\end{array}$ & - & $\begin{array}{l}120-175 \times 2-4 \mu \mathrm{m} \text {, with } 3-3.5 \mu \mathrm{m} \text { wide single } \\
\text { phialides }\end{array}$ & $\begin{array}{l}\text { Allantoid, aseptate, } 12-20 \times 2 \mu \mathrm{m} \text {, hyaline, } 1 \text { setula at the apex and } 2 \text { at } \\
\text { the base, about } 10 \mu \mathrm{m} \text { long }\end{array}$ \\
\hline
\end{tabular}

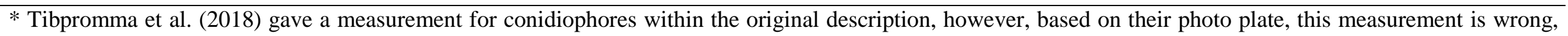
they may have muddled up conidiophores with synnema. 
Melanopsammella Höhn. was introduced by Höhnel (1919) to accommodate M. inaequalis (Grove) Höhn. (now known as Chaetosphaeria inaequalis (Grove) W. Gams \& Hol.-Jech.). All Melanopsammella species have been synonymized under the genera Chaetosphaeria and Chloridium (Réblová et al. 2016).

Sivanesan \& Chang (1997) reported a new species Chaetosphaeria ampulliformis, and linked it to Hemicorynespora multiseptata Sivan. \& H.S. Chang since the latter species was collected on the perithecial neck of $C$. ampulliformis. However, this Chaetosphaeria species and the asexual connection was uncertain and rejected by Réblová (1999) who excluded it from Chaetosphaeriaceae (Seifert et al. 2011). Hemicorynespora has been placed within Chaetosphaeriaceae in some studies (Hyde et al. 2011, Maharachchikumbura et al. 2015, 2016, Wijayawardene et al. 2017, 2018). However, no sequence data is available for Hemicorynespora. We follow the treatment by Réblová (1999) and Seifert et al. (2011) and exclude Hemicorynespora from Chaetosphaeriaceae.

Shenoy et al. (2010) performed the first phylogenetic analysis for the genus Paliphora based on LSU sequence data, and confirmed its placement within Chaetosphaeriaceae. This treatment was accepted by Seifert et al. (2011). However, Maharachchikumbura et al. (2015) and Wijayawardene et al. (2017, 2018) excluded it from Chaetosphaeriaceae, and placed Paliphora as Ascomycota genera incertae sedis. In this study, Paliphora intermedia strains (CBS 199.95 and CBS 896.97) formed a separate clade and sister to the Eucalyptostroma clade (Fig. 1), confirming the results of Crous et al. (2016, 2018). Eucalyptostroma was introduced by Crous et al. (2016) and is characterized by sporodochial, cupulate, orange-yellow conidiomata, branched and pale orangebrown conidiophores with penicillate and orange-brown conidiogenous apparatus, and producing aseptate, hyaline, smooth and ellipsoid conidia. We follow the treatment of Shenoy et al. (2010) as Paliphora has a close phylogenetic relationship with Eucalyptostroma within Chaetosphaeriaceae.

The monotypic genus Paragaeumannomyces Matsush. was established by Matsushima (2001) to accommodate $P$. sphaerocellularis Matsush. It was treated as a synonym of Chaetosphaeria since P. sphaerocellularis is identical to Chaetosphaeria raciborskii (Penz. \& Sacc.) F.A. Fernández \& Huhndorf (Lumbsch \& Huhndorf 2007a, b).

Phialogeniculata Matsush., introduced by Kobayasi (1971), was treated as a synonym of Dictyochaeta by Kuthubutheen \& Nawawi (1991a). However, this treatment was not accepted in some later studies (Matsushima 1993, Hyde et al. 1998, Seifert et al. 2011, Maharachchikumbura et al. 2015, 2016). Hyde et al. (1998) differentiated Phialogeniculata from Dictyochaeta as it lacked setae, proliferating sympodially, and possessing geniculate conidiophores, and obclavate, septate, non-setulate conidia. We maintain both genera and place Phialogeniculata within the family Chaetosphaeriaceae.

Réblová et al. (1999) accepted the sexual genus Porosphaerellopsis (with Porosphaerellopsis asexual morph) within family Chaetosphaeriaceae, and treated Porosphaeria as a synonymy of Porosphaerellopsis. Réblová (2014) synonymized Porosphaeria and Porosphaerellopsis under Sporoschismopsis, and positioned it in the Reticulascaceae (Glomerellales, Sordariomycetes) based on multi-gene phylogenetic analysis.

Réblová (1999) introduced Umbrinosphaeria Réblová to accommodate $U$. caesariata (Clinton \& Peck) Réblová with a Sporidesmium hormiscioides asexual morph. However, Subramanian (1992) had established the genus Stanjehughesia and transferred Sporidesmium hormiscioides to Stanjehughesia. Réblová et al. (2016) suggested to use Stanjehughesia rather than Umbrinosphaeria. Meanwhile, several other suggestions were provided: (1) using Chloridium rather than Gonytrichum or Melanopsammella; (2) using Menispora rather than Zignoëlla; (3) using Menisporopsis rather than Menisporopascus; (4) using Sporoschisma rather than Melanochaeta (Réblová et al. 2016).

Yang et al. (2018) studied five genera of Sordariomycetes, viz., Cryptophiale, Cryptophialoidea, Nawawia, Neonawawia and Phialosporostilbe. They confirmed the placement of Cryptophiale, Cryptophialoidea, Nawawia and Phialosporostilbe within Chaetosphaeriaceae based 
on morphological characters and phylogenetic analyses of combined LSU and ITS sequence data. However, the placements of Neonawawia in family- and order-level are uncertain.

In conclusion, we accept 49 genera (including three uncertain genera) within family Chaetosphaeriaceae and these are listed below, together with generic synonyms $\left({ }^{\mathrm{H}}=\right.$ hyphomycetes,

${ }^{\mathrm{C}}=$ coelomycetes, ${ }^{\mathrm{S}}=$ sexual genus, $?=$ uncertain classification):

\section{Chaetosphaeriaceae Réblová, M.E. Barr \& Samuels 1999}

Adautomilanezia Gusmão, S.S. Silva, Fiuza, L.A. Costa \& T.A.B. Santos $2016^{\mathrm{H}}$

Anacacumisporium Y.R. Ma \& X.G. Zhang $2016^{\mathrm{H}}$

Ascochalara Réblová $1999^{S}$

Bahusutrabeeja Subram. \& Bhat $1977^{\mathrm{H}}$

Brunneodinemasporium Crous \& R.F. Castañeda $2012^{C}$

Cacumisporium Preuss $1851^{\mathrm{H}}$

Calvolachnella Marinc., T.A. Duong \& M.J. Wingf. 2016 ${ }^{\mathrm{C}}$

Catenularia Grove $1886^{\mathrm{H}}$

= Haplochalara Linder 1933

= Psiloniella Costantin 1888

Chaetosphaeria Tul. \& C. Tul. $1863^{\mathrm{S}}$

= Aposphaeriella Died. 1912

= Chaetolentomita Maubl. 1915

= Didymopsamma Petr. 1925

= Lentomita Niessl 1876

= Melanopsammina Höhn. 1919

= Miyoshia Kawam. 1907

= Miyoshiella Kawam. 1929

= Montemartinia Curzi 1927

= Paragaeumannomyces Matsush. 2003

= Trichocollonema Höhn. 1902

= Urnularia P. Karst. 1866

?Chalarodes McKenzie $1991^{\mathrm{H}}$

Chloridium Link $1809^{\mathrm{H}}$

= Bisporomyces J.F.H. Beyma 1940

= Cirrhomyces Höhn. 1903

= Gonatotrichum Corda 1842

= Gongromeriza Preuss 1851

$=$ Gonytrichum Nees \& T. Nees 1818

= Melanopsammella Höhn. 1920

= Mesobotrys Sacc. 1880

= Monostachys G. Arnaud 1954

= Piminella G. Arnaud 1954

= Psilobotrys Sacc. 1879

= Sphaeromycetella G. Arnaud 1954

Codinaea Maire $1937^{\mathrm{H}}$

Conicomyces R.C. Sinclair, Eicker \& Morgan-Jones $1983^{\mathrm{H}}$

Craspedodidymum Hol.-Jech. $1972^{\mathrm{H}}$

Cryptophiale Piroz. $1968^{\mathrm{H}}$

Cryptophialoidea Kuthub. \& Nawawi $1987^{\mathrm{H}}$

Dendrophoma Sacc. $1880^{\mathrm{C}}$

Dictyochaeta Speg. $1923^{\mathrm{H}}$

= Menisporella Agnihothr. 1962

Dictyochaetopsis Aramb. \& Cabello 1990 ${ }^{\mathrm{H}}$

= Codinaeopsis Morgan-Jones 1976 
Dinemasporium Lév. $1846^{\mathrm{C}}$

Eucalyptostroma Crous \& M.J. Wingf. $2016^{\mathrm{H}}$

Exserticlava S. Hughes $1978^{\mathrm{H}}$

?Hyphopolynema Nag Raj $1977^{\mathrm{H}}$

Infundibulomyces Plaingam, Somrith. \& E.B.G. Jones $2003^{\mathrm{C}}$

Kionochaeta P.M. Kirk \& B. Sutton $1986^{\mathrm{H}}$

Lecythothecium Réblová \& Winka $2001^{\mathrm{S}}$

Menispora Pers. $1822^{\mathrm{H}}$

= Camptosporium Link 1818

= Ciliofusa Clem. \& Shear 1931

= Ciliofusarium Rostr. 1892

= Eriomene (Sacc.) Clem. \& Shear 1931

= Eriomenella Peyronel 1918

= Erionema Maire 1906

= Zignoëlla Sacc. 1878

Menisporopsis S. Hughes $1952^{\mathrm{H}}$

= Menisporopascus Matsush. 2003

Morrisiella Saikia \& A.K. Sarbhoy $1985^{\mathrm{H}}$

Multiguttulispora C.G. Lin \& J.K. Liu ${ }^{\mathrm{H}}$

Nawawia Marvanová $1980^{\mathrm{H}}$

?Neonawawia J. Yang, K.D. Hyde \& J.K. Liu $2018^{\mathrm{H}}$

Neopseudolachnella A. Hashim. \& Kaz. Tanaka 2015 ${ }^{\mathrm{C}}$

Paliphora Sivan. \& B. Sutton $1985^{\mathrm{H}}$

Phaeostalagmus W. Gams $1976^{\mathrm{H}}$

Phialogeniculata Matsush. $1971^{\mathrm{H}}$

Phialosporostilbe Mercado \& J. Mena $1985^{\mathrm{H}}$

Pseudodinemasporium A. Hashim. \& Kaz. Tanaka 2015 ${ }^{\mathrm{C}}$

Pseudolachnea Ranoj. 1910 ${ }^{\mathrm{C}}$

= Chaetopatella I. Hino \& Katum. 1958

= Dinemasporiella Bubák \& Kabát 1912

= Dinemasporiopsis Bubák \& Kabát 1914

Pseudolachnella Teng $1936^{\mathrm{C}}$

Pyrigemmula D. Magyar \& Shoemaker $2011^{\mathrm{H}}$

Rattania Prabhug. \& Bhat $2009^{\mathrm{H}}$

Sporoschisma Berk. \& Broome $1847^{\mathrm{H}}$

= Melanochaeta E. Müll., Harr \& Sulmont 1969

= Pithospermum Mont. 1856

Stanjehughesia Subram. $1992^{\mathrm{H}}$

= Umbrinosphaeria Réblová 1999

Striatosphaeria Samuels \& E. Müll. 1979

Tainosphaeria F.A. Fernández \& Huhndorf 2005'

Thozetella Kuntze $1891^{\mathrm{H}}$

= Thozetellopsis Agnihothr. 1958

Verhulstia Hern.-Restr. 2017 ${ }^{\mathrm{H}}$

Zanclospora S. Hughes \& W.B. Kendr. $1965^{\mathrm{H}}$

\section{Acknowledgements}

This study is supported by the Joint Fund of the National Natural Science Foundation of China and the Karst Science Research Center of Guizhou province (Grant No. U1812401), the National Natural Science Foundation of China (NSFC 31600032) and the Science and Technology Foundation of Guizhou Province (LH [2015]7061). K.D. Hyde would like to thank the Thailand Research grants entitled The future of specialist fungi in a changing climate: baseline data for 
generalist and specialist fungi associated with ants, Rhododendron species and Dracaena species (grant no: DBG6080013), Impact of climate change on fungal diversity and biogeography in the Greater Mekong Sub-region (grant no: RDG6130001). Gareth Jones is supported under the Distinguished Scientist Fellowship Program (DSFP), King Saud University, Kingdom of Saudi Arabia. We would like to thank Dr. Shaun Pennycook (Manaaki Whenua Landcare Research, New Zealand) for advising on the fungal names.

\section{References}

Agnihothrudu V. 1962 - Notes on fungi from North-east India. XVII. Menisporella assamica gen. et sp. nov. Proceedings of the Indian Academy of Sciences - Section B 56, 97-102.

Altschul SF, Gish W, Miller W, Myers EW, Lipman DJ. 1990 - Basic local alignment search tool. Journal of Molecular Biology 215, 403-410.

Arambarri A, Cabello M, Mengascini A. 1987 - Estudio sistematico de los Hyfomicetes del Rio Santiago (Prov. Buenos Aires, Argentina). Darwiniana 28, 293-301.

Burgess TI, Tan YP, Garnas J, Edwards J et al. 2019 - Current status of the Botryosphaeriaceae in Australia. Australasian Plant Pathology 48, 35-44.

Castañeda Ruíz RF, Iturriaga T, Saikawa M, Cano J, Guarro J. 2001 - The genus Menisporopsis in Venezuela with the addition of $M$. anisospora anam. sp nov from a palm tree. Cryptogamie Mycologie 22, 259-263.

Castañeda Ruíz RF, Kendrick B, Guarro J. 1998 - Notes on conidial fungi. XVII. Amphophialis, anam. gen. nov. Mycotaxon 68, 11-17.

Castresana J. 2000 - Selection of conserved blocks from multiple alignments for their use in phylogenetic analysis. Molecular Biology and Evolution 17, 540-552.

Chomnunti P, Hongsanan S, Aguirre-Hudson B, Tian Q et al. 2014 - The sooty moulds. Fungal Diversity 66, 1-36.

Crous PW, Wingfield MJ, Burgess TI, Hardy GESJ et al. 2016 - Fungal Planet description sheets: 469-557. Persoonia - Molecular Phylogeny and Evolution of Fungi 37, 218-403.

Crous PW, Wingfield MJ, Burgess TI, Hardy G et al. 2018 - Fungal Planet description sheets: 716-784. Persoonia: Molecular Phylogeny and Evolution of Fungi 40, 240-393.

Fernández FA, Huhndorf SM. 2005 - New species of Chaetosphaeria, Melanopsammella and Tainosphaeria gen. nov from the Americas. Fungal Diversity 18, 15-57.

Fernández FA, Miller AN, Huhndorf SM, Lutzoni FM, Zoller S. 2006 - Systematics of the genus Chaetosphaeria and its allied genera: morphological and phylogenetic diversity in north temperate and neotropical taxa. Mycologia 98, 121-130.

Gao JM, Xia JW, Ma YR, Li Z, Zhang XG. 2015 - Blastophragma chongqingense sp. nov. and a new record of Bahusutrabeeja angularis from southern China. Mycotaxon 130, 821-825.

Goh TK, Hyde KD. 1996 - Biodiversity of freshwater fungi. Journal of Industrial Microbiology 17, 328-345.

Goh TK, Hyde KD. 1997 - The generic distinction between Chaetopsina and Kionochaeta, with descriptions of two new species. Mycological Research 101, 1517-1523.

Goh TK, Hyde KD. 1999 - Fungi on submerged wood and bamboo in the Plover Cove Reservoir, Hong Kong. Fungal Diversity 3, 57-85.

Hall TA. 1999 - BioEdit: a user-friendly biological sequence alignment editor and analysis program for Windows 95/98/NT. Nucleic Acids Symposium Series 41, 95-98.

Hashimoto A, Sato G, Matsuda T, Matsumura M et al. 2015 - Taxonomic revision of Pseudolachnea and Pseudolachnella and establishment of Neopseudolachnella and Pseudodinemasporium gen. nov. Mycologia 107, 383-408.

Hernández-Restrepo M, Gené J, Castañeda-Ruiz RF, Mena-Portales J et al. 2017 - Phylogeny of saprobic microfungi from Southern Europe. Studies in Mycology 86, 53-97.

Hernández-Restrepo M, Schumacher RK, Wingfield MJ, Ahmad I et al. 2016 - Fungal Systematics and Evolution: FUSE 2. Sydowia 68, 193-230. 
Ho WH, Yanna, Hyde KD, Hodgkiss IJ. 2002 - Seasonality and sequential occurrence of fungi on wood submerged in Tai Po Kau Forest Stream, Hong Kong. Fungal Diversity 10, 21-43.

Höhnel F von. 1919 - Mykologische fragmente. Annales Mycologici 17, 114-133.

Hughes SJ. 1952 - Fungi from the Gold Coast. I. Mycological Papers 48, 1-91.

Hughes SJ. 1978 - New Zealand fungi 25. Miscellaneous species. New Zealand Journal of Botany 16, 311-370.

Hughes SJ, Kendrick WB. 1968 - New Zealand Fungi 12. Menispora, Codinaea, Menisporopsis. New Zealand Journal of Botany 6, 323-375.

Huhndorf SM, Miller AN, Fernandez FA. 2004 - Molecular systematics of the Sordariales: the order and the family Lasiosphaeriaceae redefined. Mycologia 96, 368-387.

Hyde K, Goh T, Steinke T. 1998 - Fungi on submerged wood in the Palmiet river, Durban, South Africa. South African Journal of Botany 64, 151-162.

Hyde KD, Goh TK. 1998 - Fungi on submerged wood in Lake Barrine, north Queensland, Australia. Mycological Research 102, 739-749.

Hyde KD, McKenzie EHC, Koko TW. 2011 - Towards incorporating anamorphic fungi in a natural classification - checklist and notes for 2010. Mycosphere 2, 1-88.

Hyde KD, Tennakoon DS, Jeewon R, Bhat DJ et al. 2019 - Fungal diversity notes 1036-1150: taxonomic and phylogenetic contributions on genera and species of fungal taxa. Fungal Diversity 96, 1-242.

Index Fungorum. [Internet]. Available from: http://www.indexfungorum.org/names/Names.asp. Accessed 22 July 2019.

Jayasiri SC, Hyde KD, Ariyawansa HA, Bhat J et al. 2015 - The Faces of Fungi database: fungal names linked with morphology, phylogeny and human impacts. Fungal Diversity 74, 3-18.

Katoh K, Standley DM. 2016 - A simple method to control over-alignment in the MAFFT multiple sequence alignment program. Bioinformatics 32, 1933-1942.

Kirk PM, Sutton BC. 1985 - A reassessment of the anamorph genus Chaetopsina (Hyphomycetes). Transactions of the British Mycological Society 85, 709-717.

Kobayasi Y. 1971 - Mycological reports from New Guinea and the Solomon Islands (1-11). Bulletin of the National Science Museum Tokyo 14, 367-551.

Kuthubutheen AJ, Nawawi A. 1988 - Two new species of Kionochaeta (Hyphomycetes) and K. ramifera from Malaysia. Transactions of the British Mycological Society 90, 437-444.

Kuthubutheen AJ, Nawawi A. 1991a - Dictyochaeta guadalcanalensis comb. nov. and several new records of the genus in Malaysia. Mycological Research 95, 1220-1223.

Kuthubutheen AJ, Nawawi A. 1991b - Key to Dictyochaeta and Codinaea species. Mycological Research 95, 1224-1229.

Larsson A. 2014 - AliView: a fast and lightweight alignment viewer and editor for large data sets. Bioinformatics 30, 3276-3278.

Li DW, Kendrick B, Chen JY. 2012 - Two new hyphomycetes: Codinaea sinensis sp. nov. and Parapleurotheciopsis quercicola sp. nov., and two new records from Quercus phillyraeoides leaf litter. Mycological Progress 11, 899-905.

Li GJ, Hyde KD, Zhao RL, Hongsanan S et al. 2016 - Fungal diversity notes 253-366: taxonomic and phylogenetic contributions to fungal taxa. Fungal Diversity 78, 1-237.

Li XX, Xia JW, Ma LG, Castañeda-Ruíz RF, Zhang XG. 2014 - A new species of Bahusutrabeeja from Guangxi, China. Mycotaxon 126, 227-230.

Liu JK, Chomnunti P, Cai L, Phookamsak R et al. 2010 - Phylogeny and morphology of Neodeightonia palmicola sp. nov. from palms. Sydowia 62, 261-276.

Liu JK, Hyde KD, Jones EBG, Ariyawansa HA et al. 2015 - Fungal diversity notes 1-110: taxonomic and phylogenetic contributions to fungal species. Fungal Diversity 72, 1-197.

Liu JK, Yang J, Maharachchikumbura SSN, McKenzie EHC et al. 2016 - Novel chaetosphaeriaceous hyphomycetes from aquatic habitats. Mycological Progress 15, 11571167.

Locquin M. 1984 - Mycologie générale et structurale. Paris, French, Masson. p. 551. 
Lu YZ, Liu KJ, Hyde KD, Bhat DJ et al. 2016 - Brunneodinemasporium jonesii and Tainosphaeria jonesii spp. nov (Chaetosphaeriaceae, Chaetosphaeriales) from southern China. Mycosphere 7, 1322-1331.

Lumbsch HT, Huhndorf SM. 2007a - Notes on ascomycete systematics. Nos. 4408-4750. Myconet 13, 59-99.

Lumbsch HT, Huhndorf SM. 2007b - Outline of ascomycota-2007. Myconet 13, 1-58.

Lumbsch HT, Huhndorf SM. 2010 - Myconet Volume 14. Part One. Outline of Ascomycota 2009. Part Two. Notes on Ascomycete Systematics. Nos. 4751-5113. Fieldiana Life and Earth Sciences, 1-64.

Luo ZL, Liu JKJ, Hyde KD, Jeewon R et al. - Freshwater Sordariomycetes Fungal Diversity (in process).

Ma YR, Xia JW, Gao JM, Li Z, Zhang XG. 2016 - Anacacumisporium, a new genus based on morphology and molecular analyses from Hainan, China. Cryptogamie Mycologie 37, 45-59.

Maharachchikumbura SSN, Hyde KD, Jones EBG, McKenzie EHC et al. 2015 - Towards a natural classification and backbone tree for Sordariomycetes. Fungal Diversity 72, 199-301.

Maharachchikumbura SSN, Hyde KD, Jones EBG, McKenzie EHC et al. 2016 - Families of Sordariomycetes. Fungal Diversity 79, 1-317.

Matsushima T. 1985 - Matsushima mycological Memoirs No. 04. Kobe, Japan, Published by the author.

Matsushima T. 1993 - Matsushima Mycological Memoirs, No. 07. Kobe, Japan, Published by the author.

Matsushima T. 2001 - Matsushima Mycological Memoirs, No. 09. Kobe, Japan, Published by the author.

Nylander J. 2004 - MrModeltest v2. Program distributed by the author. Evolutionary Biology Centre, Uppsala University, ublisher.

Page RDM. 1996 - TreeView: an application to display phylogenetic trees on personal computers. Computer Applications in the Biosciences 12, 357-358.

Perera RH, Maharachchikumbura SSN, Bhat JD, Al-Sadi AM et al. 2016 - New species of Thozetella and Chaetosphaeria and new records of Chaetosphaeria and Tainosphaeria from Thailand. Mycosphere 7, 1301-1321.

Pirozynski KA. 1968 - Cryptophiale, a new genus of Hyphomycetes. Canadian Journal of Botany 46, $1123-1127$.

Rambaut A. 2017 - [Internet]. Available from: http://tree.bio.ed.ac.uk/

Réblová M. 1999 - Studies in Chaetosphaeria sensu lato. III. Umbrinosphaeria gen. nov. and Miyoshiella with Sporidesmium anamorphs. Mycotaxon 71, 13-43.

Réblová M. 2000 - The genus Chaetosphaeria and its anamorphs. Studies in Mycology, 149-168.

Réblová M. 2014 - Sporoschismopsis angustata sp nov., a new holomorph species in the Reticulascaceae (Glomerellales), and a reappraisal of Sporoschismopsis. Mycological Progress 13, 671-681.

Réblová M, Barr M, Samuels G. 1999 - Chaetosphaeriaceae, a new family for Chaetosphaeria and its relatives. Sydowia 51, 49-70.

Réblová M, Miller AN, Rossman AY, Seifert KA et al. 2016 - Recommendations for competing sexual-asexually typified generic names in Sordariomycetes (except Diaporthales, Hypocreales, and Magnaporthales). IMA Fungus 7, 131-153.

Réblová M, Seifert KA. 2003 - Six new species of Chaetosphaeria from tropical rain forests in Thailand and redescription of Chaetosphaeria hiugensis. Sydowia 55, 313-347.

Reddy SM, Reddy SS. 1977 - A new species of Codinea. Sydowia 30, 186-188.

Ronquist F, Teslenko M, van der Mark P, Ayres DL et al. 2012 - MrBayes 3.2: efficient Bayesian phylogenetic inference and model choice across a large model space. Systematic Biology 61, 539-542.

Samuels GJ, Candoussau F, Magni JF. 1997 - Fungicolous pyrenomycetes 2. Ascocodinaea gen. nov., and reconsideration of Litschaueria. Mycologia 89, 156-162. 
Seifert KA, Morgan-Jones G, Gams W, Kendrick B. 2011 - The genera of hyphomycetes. Utrecht, The Netherlands, CBS-KNAW Fungal Biodiversity Centre.

Shenoy BD, Jeewon R, Hyde KD. 2007 - Impact of DNA sequence-data on the taxonomy of anamorphic fungi. Fungal Diversity 26, 1-54.

Shenoy BD, Jeewon R, Wang H, Amandeep K et al. 2010 - Sequence data reveals phylogenetic affinities of fungal anamorphs Bahusutrabeeja, Diplococcium, Natarajania, Paliphora, Polyschema, Rattania and Spadicoides. Fungal Diversity 44, 161-169.

Shenoy BD, Jeewon R, Wu WP, Bhat DJ, Hyde KD. 2006 - Ribosomal and RPB2 DNA sequence analyses suggest that Sporidesmium and morphologically similar genera are polyphyletic. Mycological Research 110, 916-928.

Silvestro D, Michalak I. 2012 - raxmlGUI: a graphical front-end for RAxML. Organisms Diversity \& Evolution 12, 335-337.

Sinclair RC, Eicker A, Morgan-Jones G. 1983 - Conicomyces, a unique synnematous hyphomycete genus from South Africa. Mycologia 75, 1100-1103.

Sivanesan A, Chang H. 1995 - Pseudofuscophialis lignicola gen. et sp. nov. and Chaetosphaeria capitata sp. nov. from wood in Taiwan. Mycological Research 6, 711-716.

Sivanesan A, Chang HS. 1997 - Chaetosphaeria ampulliformis sp. nov. associated with a Hemicorynespora anamorph, and a key to Hemicorynespora species. Mycological Research 101, 845-848.

Slippers B, Crous PW, Jami F, Groenewald JZ, Wingfield MJ. 2017 - Diversity in the Botryosphaeriales: Looking back, looking forward. Fungal Biology 121, 307-321.

Spegazzini C. 1923 - Algunos hongos de Tierra del Fuego. Physis Revista de la Sociedad Argentina de Ciencias Naturales 7, 9-23.

Su H, Hyde KD, Maharachchikumbura SSN, Ariyawansa HA et al. 2016 - The families Distoseptisporaceae fam. nov., Kirschsteiniotheliaceae, Sporormiaceae and Torulaceae, with new species from freshwater in Yunnan Province, China. Fungal Diversity 80, 375-409.

Subramanian CV. 1992 - A reassessment of Sporidesmium (Hyphomycetes) and some related taxa. Proceedings of the Indian Academy of Sciences (Plant Sciences) Section B 58, 179-190.

Subramanian CV. 1993 - Phialocorona pleomorpha gen. et sp. nov. and its synanamorphs. Cryptogamie Mycologie 14, 45-55.

Subramanian CV, Bhat DJ. 1977 - Bahusutrabeeja, a new genus of the hyphomycetes. Canadian Journal of Botany 55, 2202-2206.

Sun J-Z, Liu X-Z, McKenzie EHC, Jeewon R et al. 2019 - Fungicolous fungi: terminology, diversity, distribution, evolution, and species checklist. Fungal Diversity 95, 337-430.

Swofford DL. 2002 - PAUP*: Phylogenetic Analysis Using Parsimony and other methods, version 4.0 b10. Sunderland, MA: Sinauer Associates.

Tamura K, Stecher G, Peterson D, Filipski A, Kumar S. 2013 - MEGA6: Molecular Evolutionary Genetics Analysis version 6.0. Molecular Biology and Evolution 30, 2725-2729.

Tibpromma S, Hyde KD, McKenzie EHC, Bhat DJ et al. 2018 - Fungal diversity notes 840-928: micro-fungi associated with Pandanaceae. Fungal Diversity 93, 1-160.

Tsui KM, Goh TK, Hyde KD, Hodgkiss IJ. 1999 - Reflections on Menisporopsis, with the addition of M. multisetulata sp. nov. from submerged wood in Hong Kong. Mycological Research 103, 148-152.

Tulasne ELR, Tulasne C. 1863 - Selecta Fungorum Carpologia: ea documenta et icones potissimum exhibens quae varia fructuum et seminum genera in eodem fungo simul aut vicissim adesse demonstrent. Xylariei, Valsei, Sphaeriei. In imperiali typographeo. p. 1-319.

Vilgalys R, Hester M. 1990 - Rapid genetic identification and mapping of enzymatically amplified ribosomal DNA from several Cryptococcus species. Journal of Bacteriology 172, 4238-4246.

White TJ, Bruns T, Lee S, Taylor J. 1990 - Amplification and direct sequencing of fungal ribosomal RNA genes for phylogenetics. In: Innis MA, Gelfand DH, Sninsky JJ, White TJ, eds. PCR protocols: a guide to methods and applications. California, Academic Press. p. 315322. 
Whitton SR, Hyde KD, McKenzie EHC. 2000 - Dictyochaeta and Dictyochaetopsis species from the Pandanaceae. Fungal Diversity 4, 133.

Whitton SR, McKenzie EHC, Hyde KD. 2012 - Fungi Associated with Pandanaceae. Springer Netherlands.

Wijayawardene DNN, McKenzie EHC, Hyde KD. 2012 - Towards incorporating anamorphic fungi in a natural classification - checklist and notes for 2011. Mycosphere 3, 157-228.

Wijayawardene NN, Hyde KD, Lumbsch HT, Liu JK et al. 2018 - Outline of Ascomycota: 2017. Fungal Diversity 88, 167-263.

Wijayawardene NN, Hyde KD, Rajeshkumar KC, Hawksworth DL et al. 2017 - Notes for genera: Ascomycota. Fungal Diversity 86, 1-594.

Yang J, Liu NG, Liu JK, Hyde KD et al. 2018 - Phylogenetic placement of Cryptophiale, Cryptophialoidea, Nawawia, Neonawawia gen. nov. and Phialosporostilbe. Mycosphere 9, 1132-1150.

Yanna, Hyde KD. 2002 - New saprobic fungi on fronds of palms from northern Queensland, Australia. Australian systematic botany 15, 755-764. 